\title{
Measurement of DWPF Glass Viscosity - Final Report
}

by

J. R. Harbour

Westinghouse Savannah River Company

Savannah River Site

Aiken, South Carolina 29808

T. B. Edwards

R. F. Schumacher

R. J. Workman

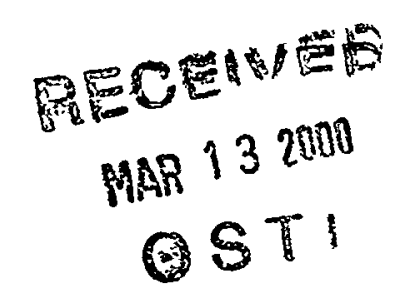

DOE Contract No. DE-AC09-96SR18500

This paper was prepared in connection with work done under the above contract number with the U.S. Department of Energy. By acceptance of this paper, the publisher and/or recipient acknowledges the U. S. Government's right to retain a nonexclusive, royalty-free license in and to any copyright covering this paper, along with the right to reproduce and to authorize others to reproduce all or part of the copyrighted paper. 
WESTINGHOUSE SAVANNAH RIVER COMPANY Savannah River Technology Center
WSRC-RP-99-01053-TL

Rev. 0

January 4,2000

M. N. Brosee, Manager

Dęfense Waste Processing Facility

Attention: W. D. Kerley, 704-S

Task Initiator: R. E. Edwards, 704-25S

\section{MEASUREMENT OF DWPF GLASS VISCOSITY - FINAL REPORT (U)}

The attached report details the results of a scoping study funded by the DWPF for the measurement of melt viscosities for simulated glasses representative of Macrobatch 2 (Tank 42/51 feed). The driver for this task was the fact that Macrobatch 2 glasses had predicted viscosities very close to the upper limit of 100 poise at $1150^{\circ} \mathrm{C}$.

A significant accomplishment of this work was the establishment of an in-house capability for measuring glass viscosities at $1150^{\circ} \mathrm{C}$. This Harrop, high-temperature viscometer measures viscosity as a function of temperature with a very small amount of glass ( $\sim 6$ grams). This enables SRTC, and consequently DWPF, to obtain viscosity data within a reasonable time for relatively low cost. Several WCP glasses were measured and compared with results from previous measurements by Sharp-Shurtz. In addition, CELS (Corning) and PNNL measured viscosities of some of these glasses as part of this study. The results revealed that SRTC's measurements were $\sim 10 \%$ higher than measurements by CELS and Sharp-Shurtz, but close to those of PNNL. With this knowledge, it is now possible to measure glass melt viscosities inhouse and obtain useful, approximate values of melt viscosity at $1150^{\circ} \mathrm{C}$.

The limited results obtained from this study on simulated Macrobatch 2 glasses (with and without uranium) reveal that uranium may increase melt viscosity in this compositional region. For uranium-bearing glasses, predicted viscosities from the measured compositions appear to be less than the measured viscosity values, whereas for glasses without uranium, the predicted viscosity tends to be less than measured. PNNL and CELS measurements support SRTC's results with these glasses (PNNL measured the uranium containing glasses while CELS measured the glasses without uranium). Further work will be required to determine whether this effect is real.

If you have any questions about the results contact J. R. Harbour at 5-8725 or T. B. Edwards at 5-5148.

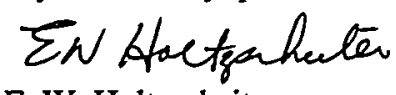

E. W. Holtzscheiter

Immobilization Technology Section 
WSRC-RP-99-01053

Revision 0

\section{DISCLAIMER}

This report was prepared as an account of work sponsored by an agency of the United States Government. Neither the United States Government nor any agency thereof, nor any of their employees, makes any warranty, express or implied, or assumes any legal liability or responsibility for the accuracy, completeness, or usefulness of any information, apparatus, product or process disclosed, or represents that its use would not infringe privately owned rights. Reference herein to any specific commercial product, process or service by trade name, trademark, manufacturer, or otherwise does not necessarily constitute or imply its endorsement, recommendation, or favoring by the United States Government or any agency thereof. The views and opinions of authors expressed herein do not necessarily state or reflect those of the United States Government or any agency thereof.

This report has been reproduced directly from the best available copy.

Available for sale to the public, in paper, from: U.S. Department of Commerce, National Technical Information Service, 5285 Port Royal Road, Springfield, VA 22161, phone: (800) 553-6847

fax: (703) 605-6900

email: orders@ntis.fedworld.gov

online ordering: http://www.ntis.gov/ordering.htm

Available electronically at http://www.doe.gov/bridge

Available for a processing fee to U.S. Department of Energy and its contractors, in paper, from: U.S. Department of Energy, Office of Scientific and Technical Information, P.O. Box 62, Oak Ridge, TN 37831-0062, phone: (865) 576-8401

fax: (865) 576-5728

email: reports@adonis.osti.gov 


\section{DISCLAIMER}

Portions of this document may be illegible in electronic image products. Images are produced from the best available original document. 
WSRC-RP-99-01053

Revision 0 


\section{INTRODUCTION}

This report details the results of a scoping study funded by the Defense Waste Processing Facility (DWPF) for the measurement of melt viscosities for simulated glasses representative of Macrobatch 2 (Tank 42/51 feed). The driver for this task was the fact that Macrobatch 2 glasses had predicted viscosities very close to the upper limit of 100 Poise $[1,2]$. Therefore, one objective of this scoping study was to gain insight into the adequacy of the viscosity model in the compositional region of interest for Macrobatch 2. As part of this objective, glasses were fabricated using depleted uranium to determine whether uranium has an effect on glass viscosity at $1150^{\circ} \mathrm{C}$. Uranium is not considered in the current viscosity model.

A second, major objective of this task was to determine the viability of developing a measurement technique for glass viscosity at SRTC. The benefits of establishing this measurement capability at SRTC are (1) a faster turnaround of viscosity results (2) lower cost for the data by keeping the work scope within SRTC, and (3) use of only a small amount of glass ( $\sim 6$ grams) for each measurement. The small sample size is important at the bench-scale experimental level since it enables SRTC to measure many more glass samples, thereby improving the quality of the results and conclusions. ${ }^{1}$

The results of the initial viscosity measurements using the SRTC hightemperature viscometer were included in an Interim Report [3], and these values are repeated here for completeness. This Final Report focuses on (1) the quality of the data generated at SRTC and (2) on the comparison of the SRTC viscosity results with recent results from Corning (CELS) and the Pacific Northwest National Laboratory (PNNL) and with previous results from Sharp-Shurtz (SS). The CELS, PNNL, and SRTC results were determined using samples of the same batch glasses.

\section{RESULTS}

SRTC Viscometer. The setup and establishment of a Harrop, high-temperature viscometer at SRTC has been described in an internal report [4]. This unit uses approximately 6 grams of glass, which is considerably less than the amount used by CELS and PNNL. CELS required 700 grams per viscosity measurement and PNNL required 200 grams for each viscosity measurement. Two crucible/spindle sets (Set A and Set B) were used for these measurements. This allowed for one viscosity measurement per day, since it takes $\sim 1$ day to clean the crucible and spindle. Set A was calibrated using the NIST 717a borosilicate glass standard. This NIST glass is significantly more viscous than DWPF-type glasses with a viscosity close to 2000 Poise at $1200^{\circ} \mathrm{C}$. For comparison, the normal range for DWPF glasses is from 20 to 100 Poise at $1150^{\circ} \mathrm{C}$.

\footnotetext{
Smaller samples are of particular advantage when working with uranium-bearing glasses.
} 
Due to the high viscosity of the NIST 717a glass, it was decided to measure the viscosities of the HM, Purex, and Batch 1 WCP glasses. The melt viscosities for these glasses had been previously measured by SS, thereby providing an independent measure of glass viscosity for glasses in the range of interest for DWPF.

Experimental. The melt viscosity was measured according to GTOP-3-111, Rev.0. The glass density for each sample was determined since a constant volume of glass is required in the crucible for each glass sample. The mass of glass to be added to the crucible is $2.6 \mathrm{cc} \cdot$ glass density in $(\mathrm{g} / \mathrm{cc})$. Viscosity measurements were made at 5 temperatures starting with a temperature of $1190^{\circ} \mathrm{C}$. The temperature was lowered in $\sim 50^{\circ} \mathrm{C}$ intervals and, after equilibration at each temperature, another measurement was made.

Volatilization and crystallization can affect the viscosity results. For example, volatilization of a compound that reduces viscosity will lead to an increase in viscosity with time. On the other hand, crystallization in the melt has the potential to increase the measured viscosity. Starting the viscosity experiment at temperatures above the liquidus temperature minimizes the effect of crystallization on glass viscosity. The impact of volatile species can be determined by measuring the viscosity as a function of time or by choosing a thermal profile that allows for detection of hysteresis effects.

A detailed determination of the impact of volatilization and crystallization on the measured viscosity was beyond the scope of this task. Rather, a comparison of the results from PNNL, Corning, and SRTC was used to demonstrate that consistent results could be obtained. Crystallization does not significantly impact the Fulcher fit for these data since the viscosity change with temperature is relatively small in the region of $1150^{\circ} \mathrm{C}$. Therefore, even if crystallization occurred, its impact on the measured viscosity at $1150^{\circ} \mathrm{C}$ would be small. PNNL followed a sequence of temperatures that allows for hysteresis effects on viscosity due to volatility to be observed. If the PNNL and SRTC results are close, it may be assumed that no significant volatility occurred with these glasses during the viscosity measurements.

Viscosity Measurements. The viscosities at a series of temperatures were calculated from the rpm's of the spindle, the \%torque on the spindle, and a constant, $\mathrm{K}$, measured from the NIST 717 a glass. The $\mathrm{K}$ constant actually varied slightly with temperature, and for temperatures between 1050 and $1500{ }^{\circ} \mathrm{C}$ it followed the form: $198.4-\left(0.063454 \times \mathrm{T}\right.$ in $\left.{ }^{\circ} \mathrm{C}\right) .^{2}$ Using this equation, the viscosity at a given measurement temperature was then calculated by equation (1):

$$
\text { Viscosity (Poise) } \eta=[\% \text { torque } / \mathrm{RPM}] \cdot \mathrm{K}
$$


All of the viscosity measurements generated by SRTC are provided in Exhibit A.1 of the Appendix. The viscosity (in Poise) of each of these glasses at $1150^{\circ} \mathrm{C}$ is to be estimated from a Fulcher equation fitted to these measurements. The functional form of the (three-parameter) Fulcher equation (expressed in Poise) used to fit these data is given by equation (2):

$$
\ln \hat{\eta}=A+\frac{B}{(T-C)}
$$

where $\ln \hat{\eta}$ represents the natural logarithm of the estimated viscosity (in Poise), $\hat{\eta}$, and $A, B$, and $C$ represent the parameters of the model that were determined from the available measurements (expressed in Poise) at various temperatures (represented by $\mathrm{T}$, in degrees Celsius). The fitted model was then used to predict the viscosity of the given glass at $1150^{\circ} \mathrm{C}$.

Exhibit A.2 in the Appendix provides the results from such a Fulcher fit for each set of viscosity measurements presented in Exhibit A.1. Exhibits A.3-A.5 provide the results of the Fulcher fits to the viscosity measurements from PNNL, CELS, and SS, respectively.

Repeatability. The Batch 1 glass, with a viscosity close to 50 Poise at $1150^{\circ} \mathrm{C}$ (see Table 5), was chosen as the secondary standard for this work and for viscosity measurements of glasses produced during the glass studies for salt disposition. A number of measurements were made at SRTC on the Batch 1 glass as a function of time and the results are presented in Table 1 and Figure 1. These results provide a measure of the reproducibility of the technique.

Table 1. SRTC Batch 1 Viscosities at $1150^{\circ} \mathrm{C}$ (Poise) from Fulcher Equations

\begin{tabular}{|c|c|c|c|}
\hline Date & $\begin{array}{c}\text { Crucible/ } \\
\text { Spindle }\end{array}$ & $\begin{array}{c}\eta_{1150} \\
\text { Viscosity at } 1150^{\circ} \mathbf{C} \text { (Poise) }\end{array}$ & $\cdot \ln \left(\eta_{1150}\right)$ \\
\hline $2 / 9 / 99$ & A & 48.6 & 3.884 \\
\hline $2 / 17 / 99$ & A & 49.7 & 3.906 \\
\hline $4 / 30 / 99$ & B & 46.4 & 3.837 \\
\hline $6 / 8 / 99$ & A & 48.9 & 3.890 \\
\hline $6 / 9 / 99$ & B & 47.3 & 3.857 \\
\hline $8 / 19 / 99$ & A & 49.9 & 3.910 \\
\hline $8 / 31 / 99$ & B & 49.5 & 3.902 \\
\hline
\end{tabular}

There is no indication of a statistical difference in the viscosities presented in Table 1 due to the crucible/spindle set used. The relative standard deviation of these data (expressed as a percentage) is $2.7 \%$, a (1-sigma) measure of the repeatability of the SRTC measurement process.

Figure 1 also presents the repeatability data by providing a plot of the Fulcher fits for all of the SRTC Batch 1 measurements. 
Figure 1. Graphical Comparison of SRTC Batch 1 Viscosities

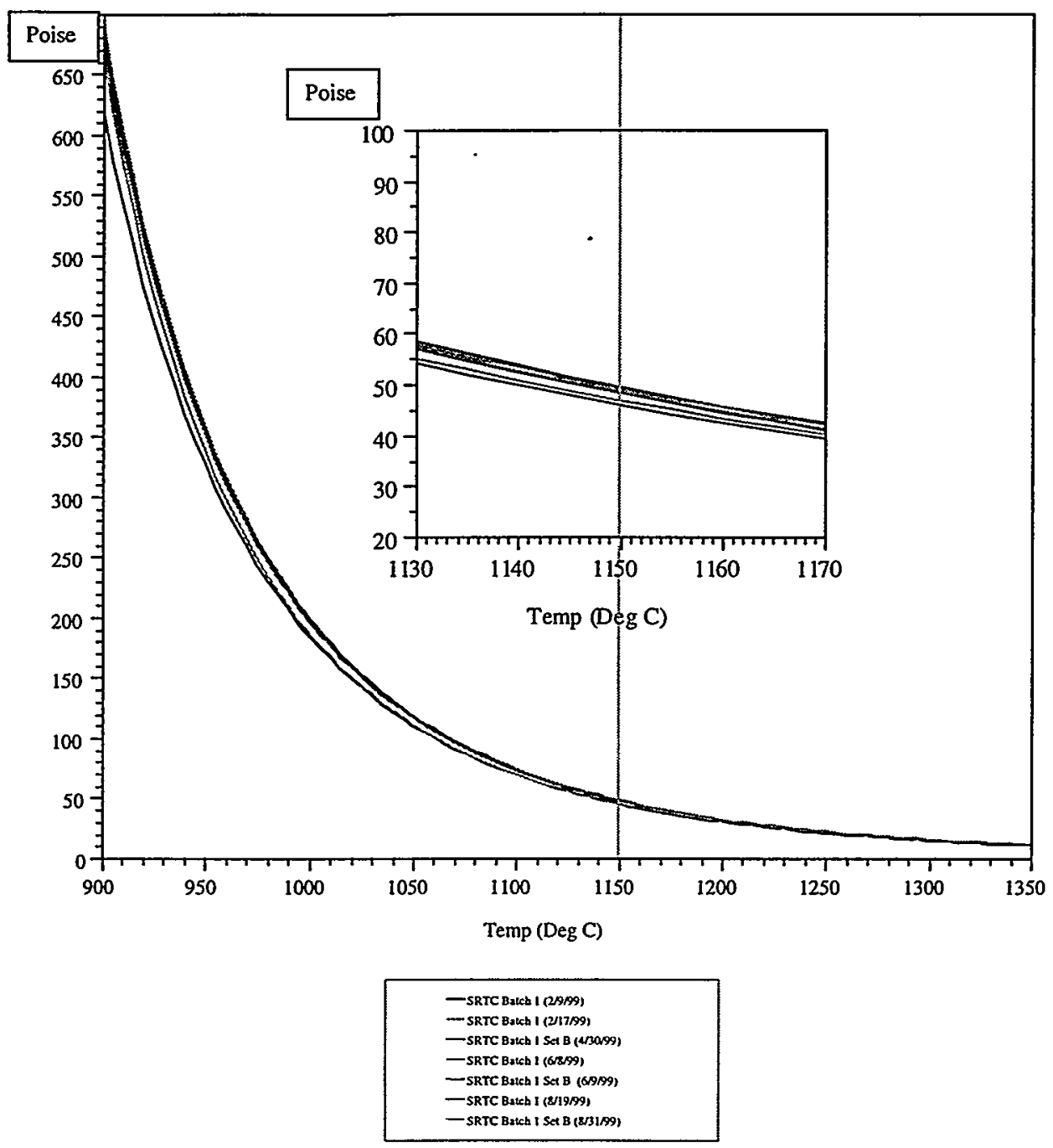

Comparisons. In this section, comparisons are made between the SRTC results and those obtained by PNNL, CELS, and/or Sharp-Shurtz (SS). Figure 2 provides a plot of the SRTC values for Batch 1 along with a previous measured set of SS results for this glass. 
Figure 2. Graphical Comparison of Batch 1 Viscosities (SRTC and SS)

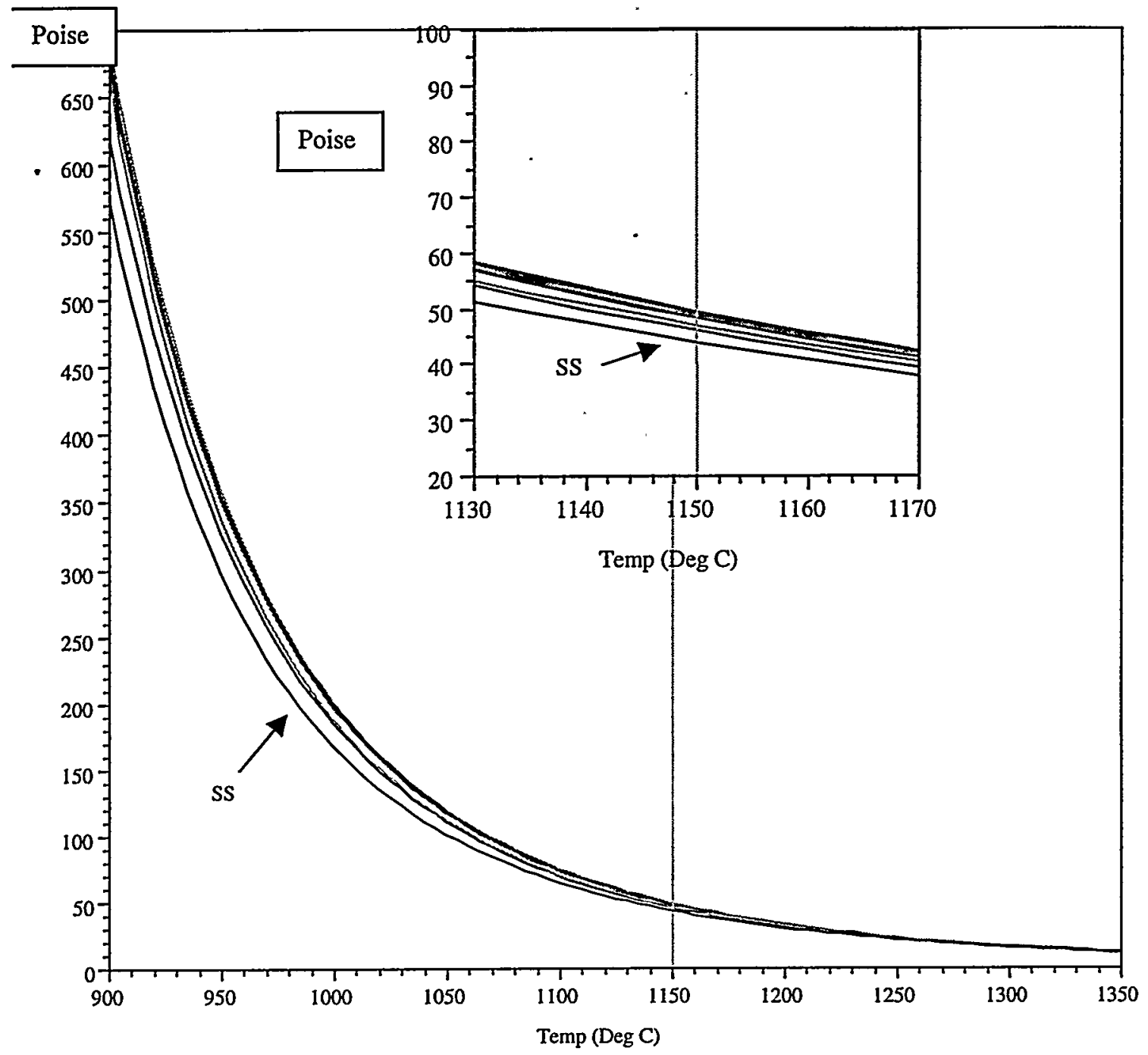

- SRTC Batch 1 (2/9/99)

- SRTC Batch $1(2 / 17 / 99)$

- SRTC Batch 1 Set B (4/30/99)

- SRTC Batch 1 (6/8/99)

- SRTC Batch 1 Set B (6/9/99)

- SRTC Batch 1 (8/19/99)

- SRTC Batch 1 Set B $(8 / 31 / 99)$

-SS Batch 1

At $1150^{\circ} \mathrm{C}$, the average SRTC result is 48.6 Poise and the Sharp-Shurtz result is 44.2 Poise, a difference of $9.9 \%$ for the Batch 1 glass. When compared using a (natural) logarithm scale, the difference is only $2.5 \%$.

. For this task the melt viscosity for the HM glass was measured by CELS, SRTC, and PNNL as a function of temperature. These results are summarized in Figure 3 and Table 2 and include a previous HM viscosity measurement by SS. 
Figure 3. Graphical Comparison of HM Viscosities

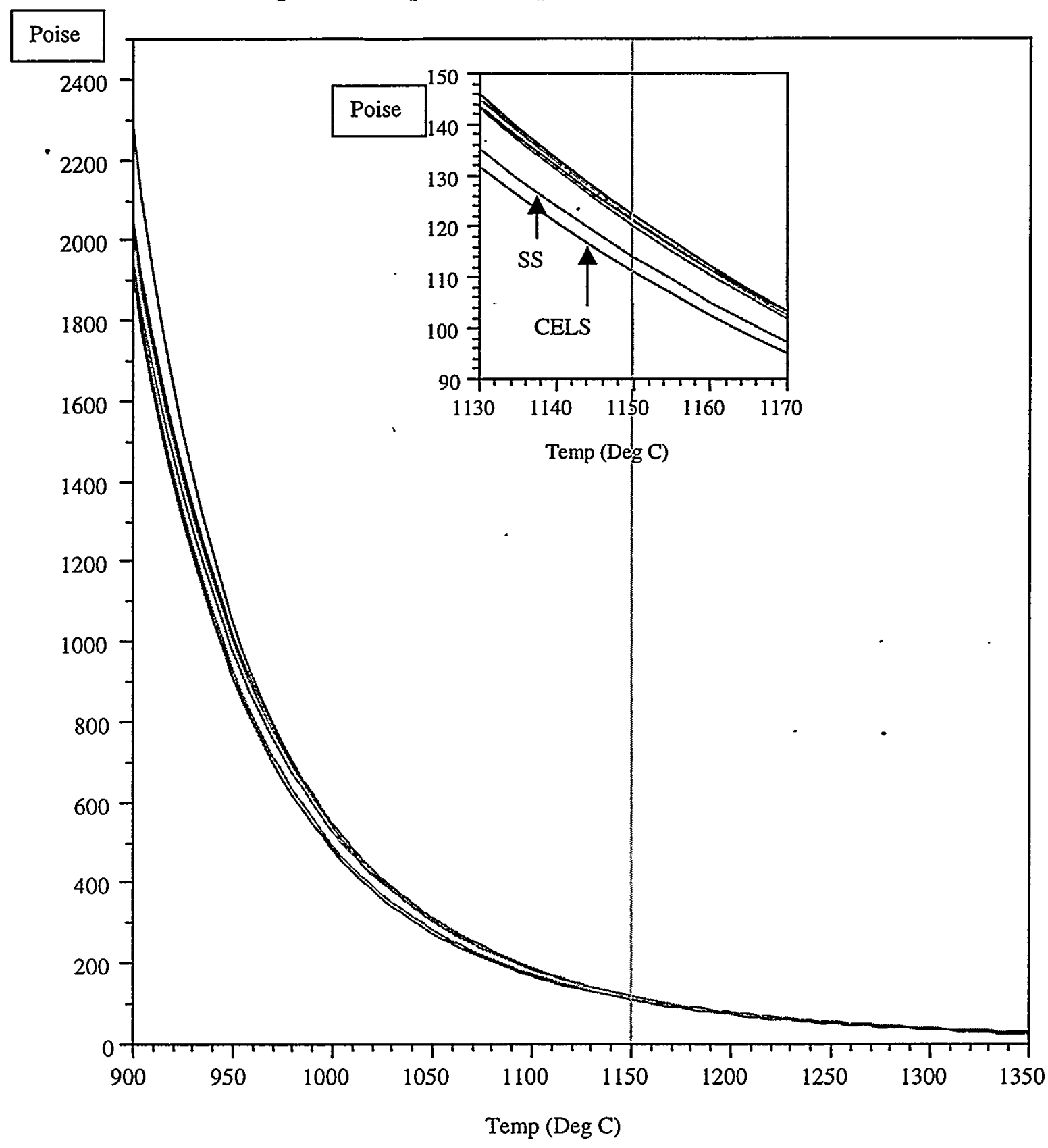

-PNNL HM

- SRTC HM Set A (1/22/99)

- SRTC HM Set A (1/28/99)

- SRTC HM Set A (4/7/99)

- SRTC HM Set B (4/28/99)

- CELS HM

-SS HM 
Table 2. HM Viscosities from the Fulcher Fits

\begin{tabular}{|c|c|c|}
\hline Organization & $\begin{array}{c}\eta_{1150} \\
\text { Viscosity at } 1150^{\circ} \mathbf{C}(\text { Poise) }\end{array}$ & $\operatorname{In}\left(\eta_{1150}\right)$ \\
\hline CELS & 111.5 & 4.714 \\
\hline PNNL & 121.5 & 4.800 \\
\hline SRTC (Set A; $1 / 22 / 99)$ & 121.8 & 4.802 \\
\hline SRTC (Set A; $1 / 28 / 99)$ & 122.6 & 4.809 \\
\hline SRTC (Set A; $4 / 7 / 99)$ & 121.9 & 4.803 \\
\hline SRTC (Set B; $4 / 28 / 99)$ & 120.6 & 4.792 \\
\hline SS & 114.4 & 4.740 \\
\hline
\end{tabular}

The average SRTC value is 121.7 Poise: $9.1 \%$ higher than the CELS value, $0.2 \%$ higher than the PNNL value, and 6.4\% higher than the Sharp-Shurtz value. In log-space, the differences are: SRTC is $1.9 \%$ higher than CELS, $0.03 \%$ higher than PNNL, and $1.3 \%$ higher than SS.

Figure 4 provides a similar plot of viscosities for the Purex glass, measured by SRTC and SS. 
Figure 4. Graphical Comparison of Purex Viscosities (from Fulcher Fits)

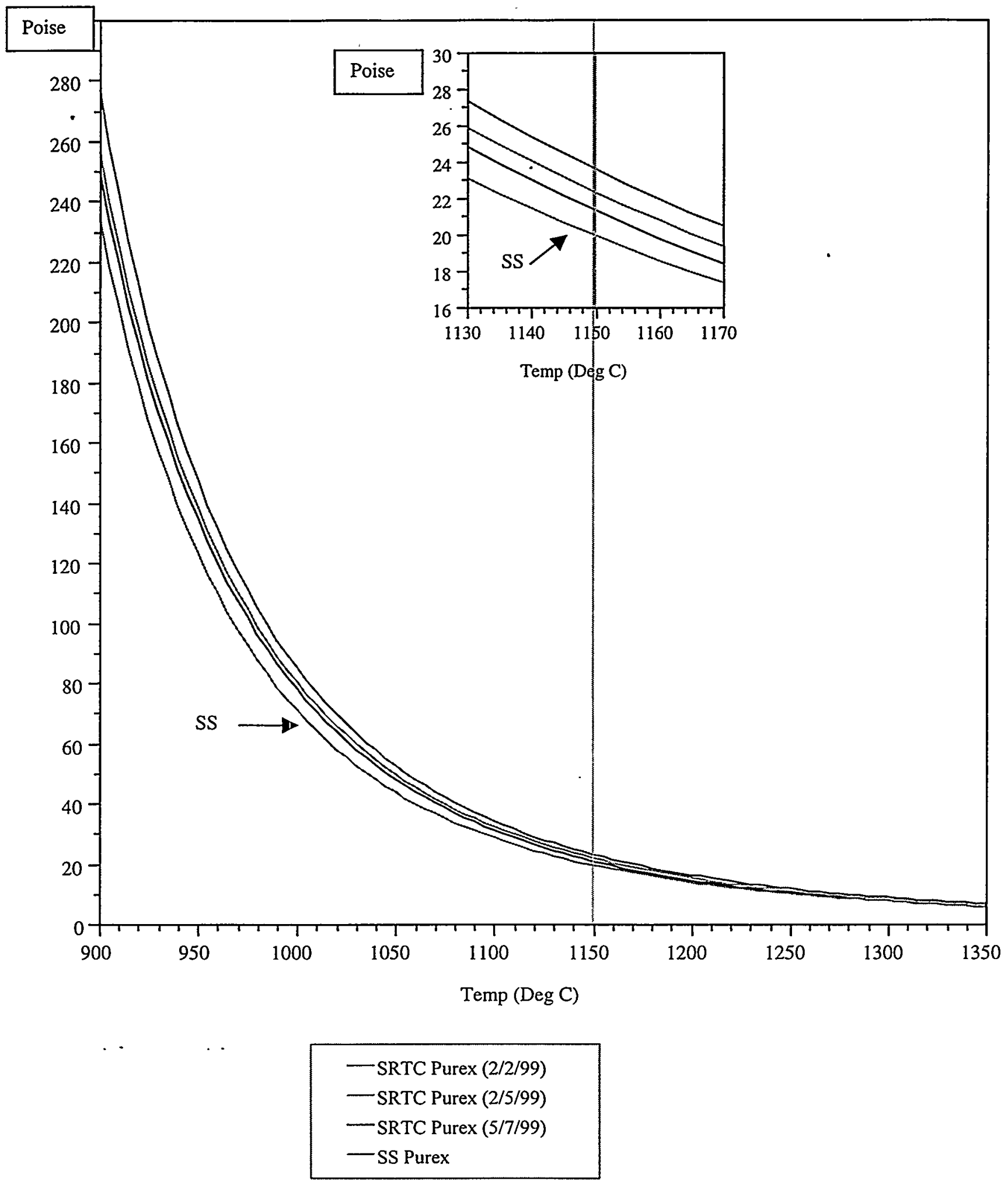

The average SRTC value is 22.5 Poise, $12.5 \%$ higher than the SS value of 20.0 Poise for Purex. In log-space, SRTC is $0.6 \%$ higher than SS. 
Uranium Glasses. The three uranium-containing glasses included in this task were sent to PNNL for viscosity measurements. This was done to provide independent verification of the SRTC results. The CELS would not accept these depleted uranium glasses for measurement of viscosity due to the radioactivity. The results are shown in Table 3 and Figures 5, 6 and 7.

Table 3. Measured Viscosities (from the Fulcher fits) for the Three Uranium-Containing Glasses

\begin{tabular}{|c|c|c|c|}
\hline $\begin{array}{c}\text { Glass } \\
\text { D }\end{array}$ & Organization & $\begin{array}{c}\eta_{11150} \\
\text { Viscosity at } \\
1150^{\circ} \mathrm{C} \text { (Poise) }\end{array}$ & $\ln \left(\mathfrak{\eta}_{1150}\right)$ \\
\hline$\overline{1}$ & PNNL & 88.8 & 4.486 \\
\hline$\overline{1}$ & SRTC (4/12/99) & 88.5 & 4.483 \\
\hline 1 & SRTC $(5 / 4 / 99)$ & 87.3 & 4.469 \\
\hline 3 & PNNL & 95.8 & 4.562 \\
\hline 3 & SRTC (3/31/99) & 98.4 & 4.589 \\
\hline 3 & SRTC (4/19/99) & $\overline{107.4}$ & 4.677 \\
\hline 3 & SRTC (5/11/99) & 101.6 & 4.621 \\
\hline 5 & PNNL & 104.7 & 4.651 \\
\hline 5 & SRTC (4/15/99) & 107.4 & 4.677 \\
\hline 5 & SRTC (4/27/99) & 109.4 & 4.695 \\
\hline
\end{tabular}

For Glass 1 , the average SRTC viscosity at $1150^{\circ} \mathrm{C}$ is 87.9 Poise, $1.0 \%$ lower than the PNNL value of 88.1 Poise. For Glass 3, the average SRTC viscosity at $1150^{\circ} \mathrm{C}$ is 102.5 Poise, $7.0 \%$ higher than the PNNL value of 95.8 Poise. For Glass 5 , the average SRTC viscosity at $1150^{\circ} \mathrm{C}$ is 108.4 Poise, $3.5 \%$ higher than the PNNL value of 104.7 Poise.

In log-space, the differences are: for Glass 1 , SRTC is $0.2 \%$ lower than PNNL, for Glass 3, SRTC is $1.5 \%$ higher than PNNL, and for Glass 5, SRTC is $0.7 \%$ higher than PNNL. 
Figure 5. Graphical Comparison of Glass 1 Viscosities (A Uranium-Bearing Glass)

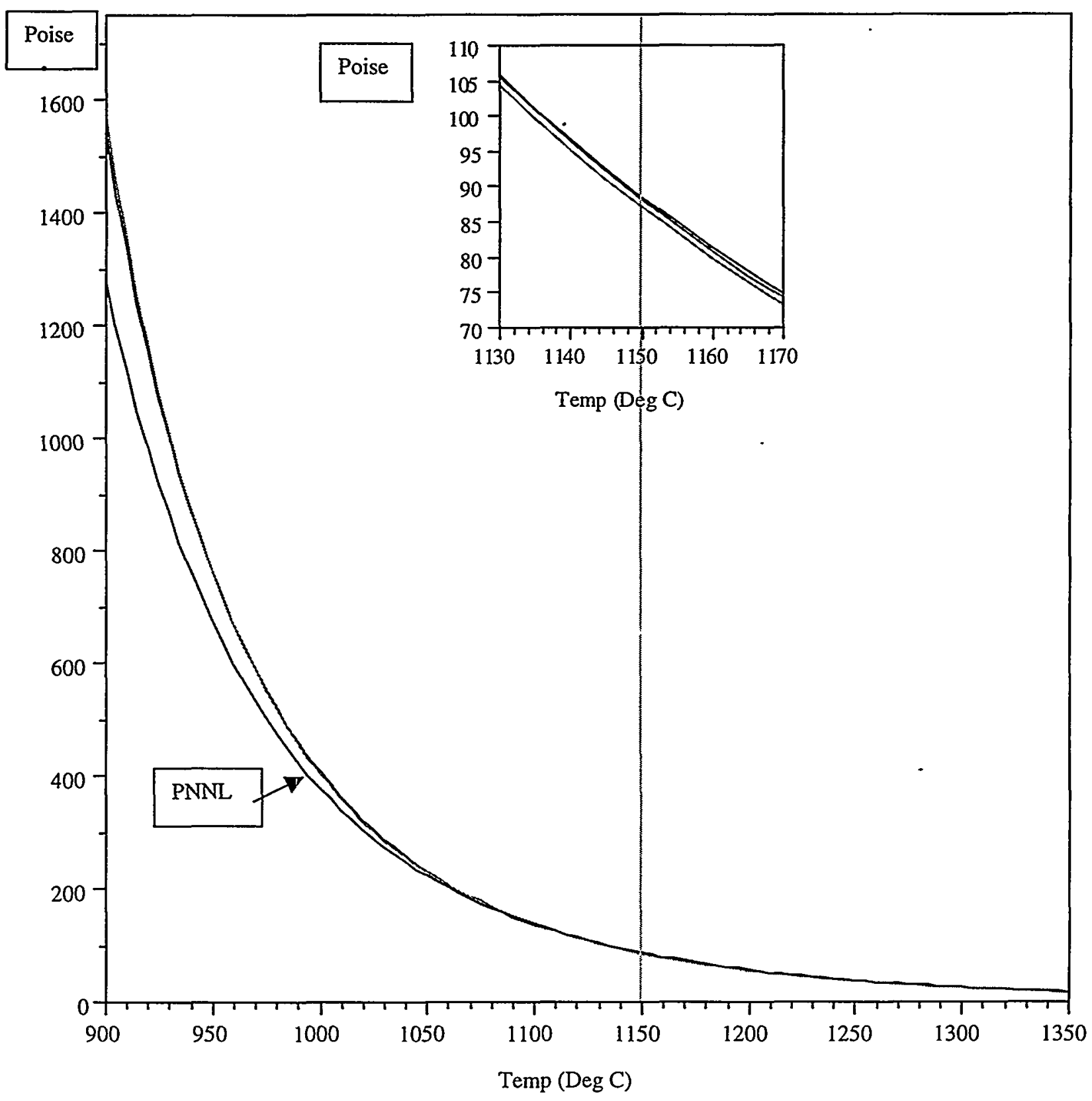

- SRTC Glass 1 (4/12/99)

- SRTC Glass 1 (5/4/99)

-PNNL Glass 1 
Figure 6. Graphical Comparison of Glass 3 Viscosities

(A Uranium-Bearing Glass)

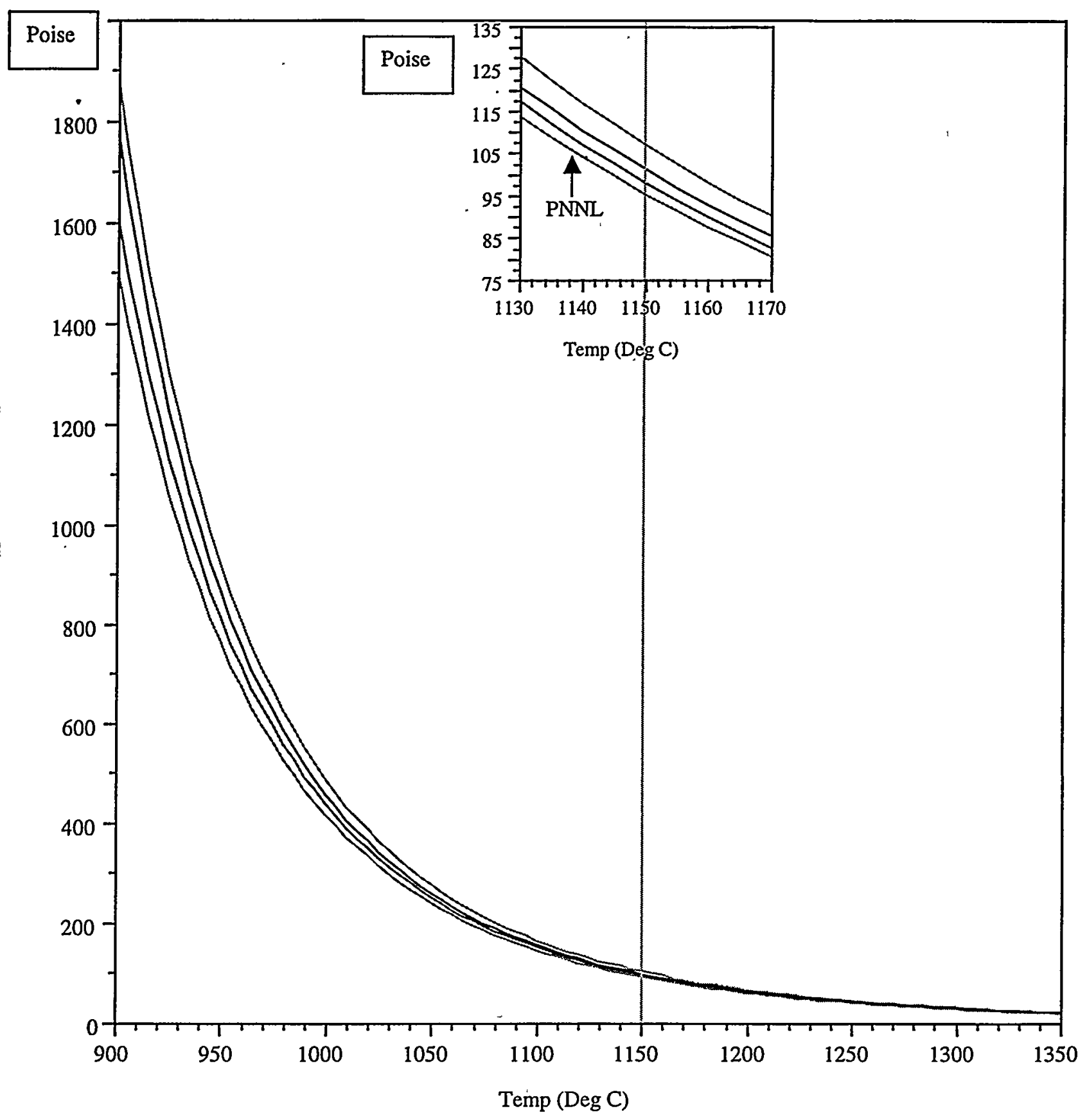

- SRTC Glass 3 (3/31/99)

- SRTC Glass 3 (4/19/99)

- SRTC Glass 3 (5/1 1/99)

-PNNL Glass 3 
Figure 7. Graphical Comparison of Glass 5 Viscosities

(A Uranium-Bearing Glass)

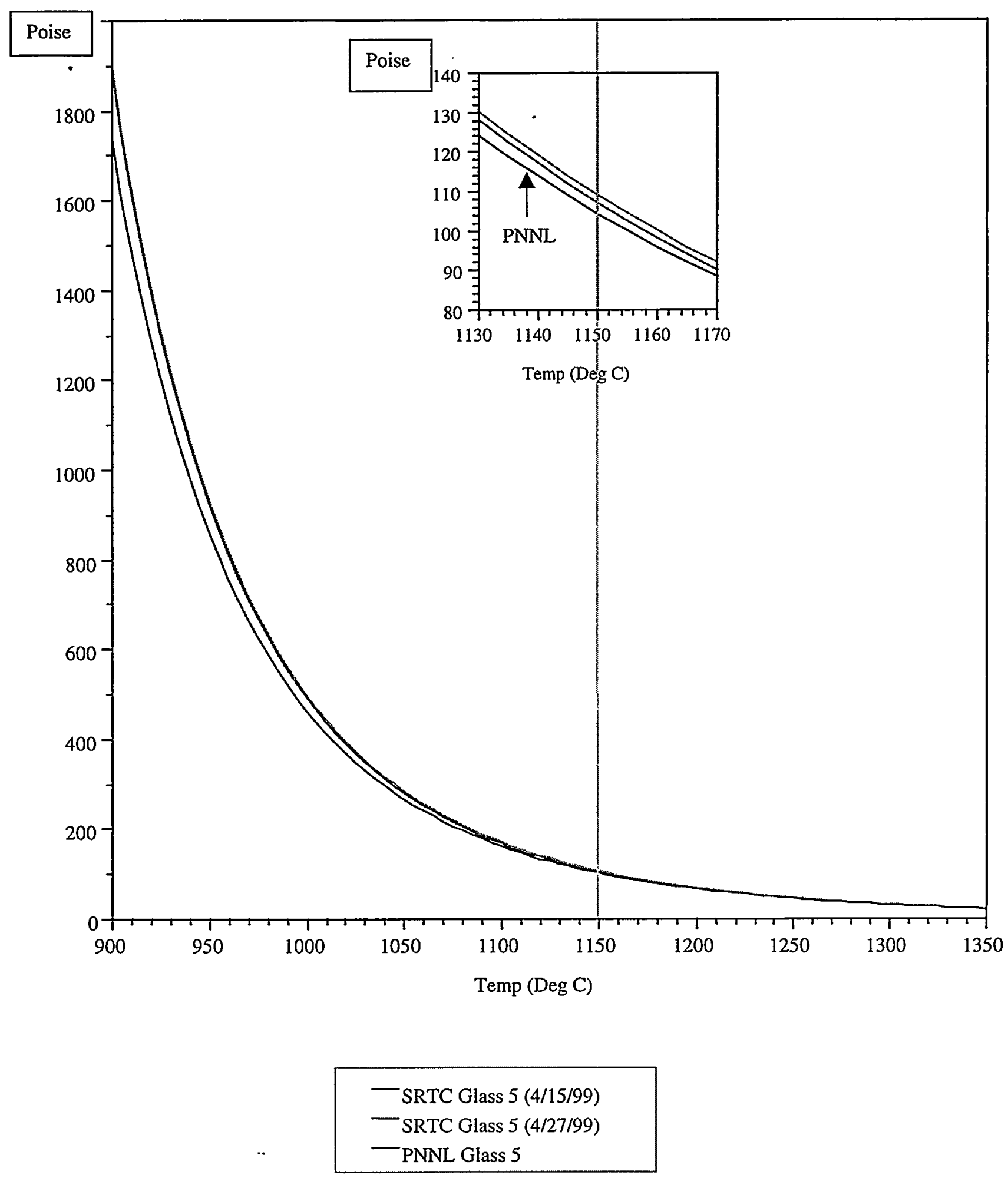


Other Glasses. Two other glasses (designated as Glass 2 and Glass 4) were included in this task. These glasses did not contain uranium, and they were sent to CELS for viscosity measurements. This was done to provide independent verification of the SRTC results for these glasses. The results are shown in Table 4 and Figures 8, and 9.

Table 4. Measured Viscosities (from the Fulcher fits) for Glass 2 and Glass 4

\begin{tabular}{|c|c|c|c|}
\hline $\begin{array}{c}\text { Glass } \\
\text { ID }\end{array}$ & Organization & $\begin{array}{c}\eta_{1150} \\
\text { Viscosity at } \\
1150^{\circ} \mathrm{C} \text { (Poise) }\end{array}$ & $\ln \left(\eta_{1150}\right)$ \\
\hline 2 & CELS & 61.2 & 4.114 \\
\hline 2 & SRTC (2/24/99) & 65.9 & 4.188 \\
\hline 2 & SRTC (3/4/99) & 65.3 & 4.179 \\
\hline 4 & CELS & 92.8 & 4.530 \\
\hline 4 & SRTC (3/2/99) & 102.3 & 4.628 \\
\hline 4 & SRTC (3/9/99) & 102.4 & 4.629 \\
\hline
\end{tabular}

For Glass 2, the average SRTC viscosity at $1150{ }^{\circ} \mathrm{C}$ is 65.6 Poise, $7.2 \%$ above the CELS value of 61.2 Poise. For Glass 4 , the average SRTC viscosity at $1150^{\circ} \mathrm{C}$ is 102.4 Poise, $10.3 \%$ higher than the CELS value of 92.8 Poise.

In log-space, the differences are: for Glass 2, SRTC is $1.7 \%$ higher than CELS and for Glass 4, SRTC is 2.2\% higher than CELS. 
Revision 0

Figure 8. Graphical Comparison of Glass 2 Viscosities

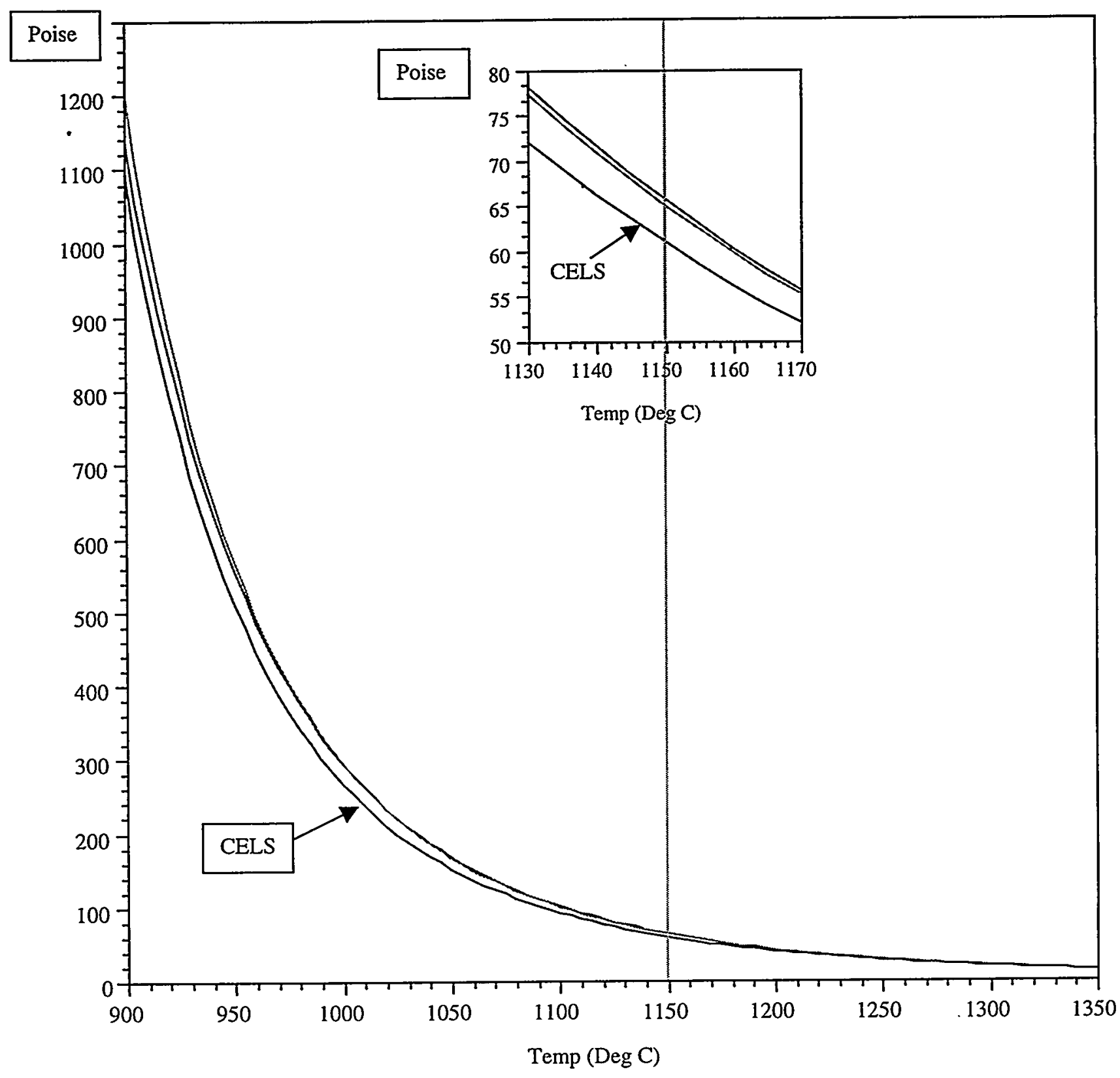

- SRTC Glass 2 (3/4/99)

-CELS Glass 2 
Figure 9. Graphical Comparison of Glass 4 Viscosities

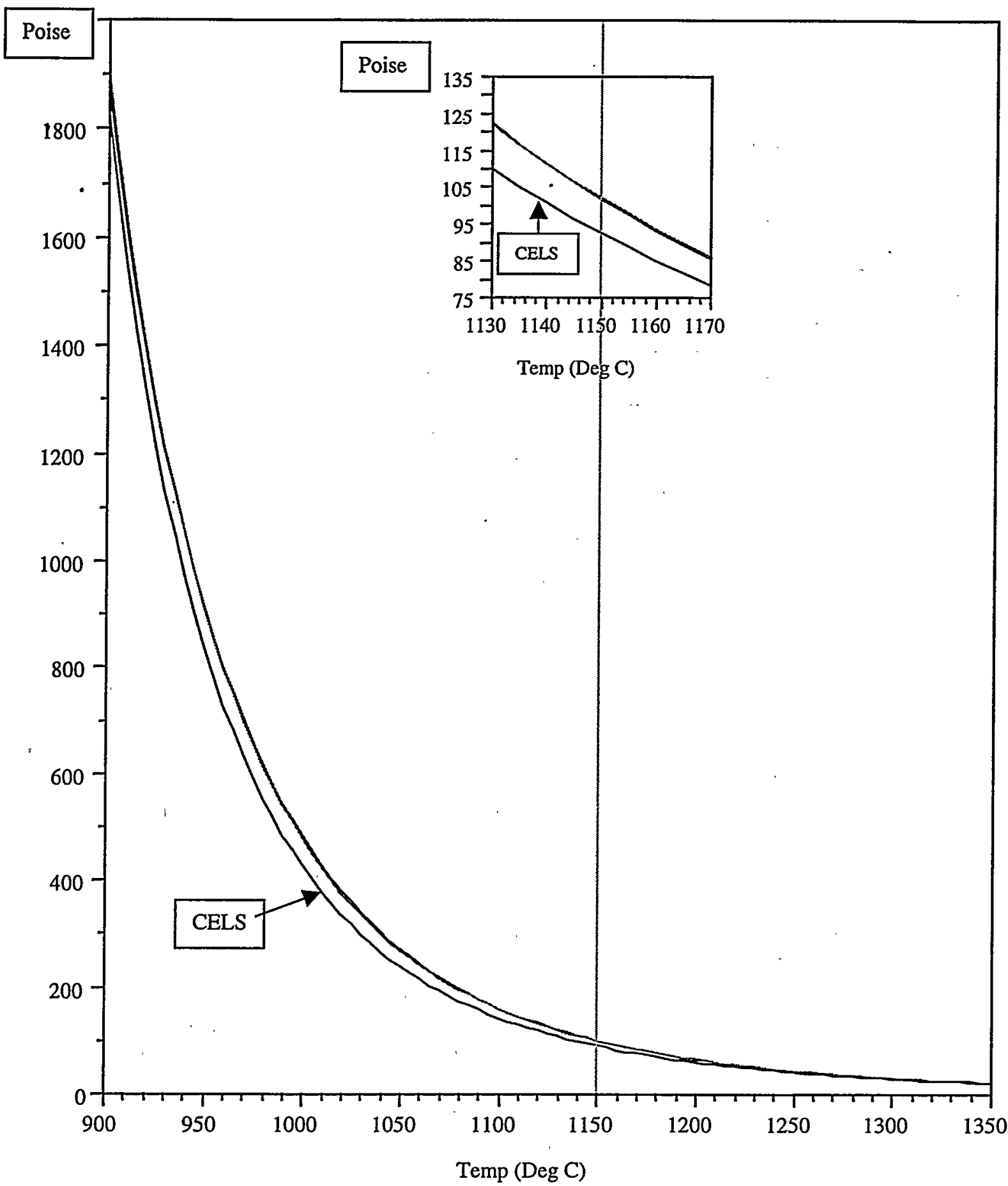

- - SRTC Glass $4(3 / 2 / 99)$
- SRTC Glass $4(3 / 9 / 99)$
- CELS Glass 4


Viscosity Model Predictions. Exhibit A.5 in the Appendix provides the viscosity predictions for the glasses considered in this study determined from the DWPF model for this property, which relates viscosity at $1150^{\circ} \mathrm{C}$ to glass composition [5]. Viscosity predictions for the glasses are provided using target (or reference) - and measured (where available) 'compositions. Information from Exhibit A.5 is summarized in Table 5.

Table 5: Summary of Viscosity Measurements and Predictions (in Poise) at $1150^{\circ} \mathrm{C}$ Based on Target and Measured Compositions of the Glasses

\begin{tabular}{|c|c|c|c|c|c|c|}
\hline & \multicolumn{2}{|c|}{ Predicted Viscosity } & \multicolumn{5}{c|}{ Measured Viscosity $^{2}$} \\
\hline Glass & Target & Measured & & CELS & SS \\
\hline ID & Composition & Composition & SRTC & PNNL & CEL \\
\hline Glass 1 (U) & 82.5 & 63.1 & 87.9 & 88.8 & - & - \\
\hline Glass 2 & 82.5 & 94.0 & 65.6 & - & 61.2 & - \\
\hline Glass 3 (U) & 92.9 & 82.9 & 102.5 & 95.8 & - & - \\
\hline Glass 4 & 108.9 & 119.8 & 102.4 & - & 92.6 & - \\
\hline Glass 5 (U) & 108.9 & 92.7 & 108.4 & 104.7 & - & - \\
\hline Batch 1 & 56.2 & - & 48.6 & - & - & 44.2 \\
\hline HM & 137.2 & - & 121.7 & 121.5 & 111.5 & 114.4 \\
\hline Purex & 24.8 & - & 22.5 & - & - & 20.0 \\
\hline Uranium Std & 33.2 & - & 32.3 & - & - & - \\
\hline
\end{tabular}

The results for the five glasses, denoted as Glass 1 through Glass 5, representing Macrobatch 2 (with and without uranium) are of primary interest. The PNNL and SRTC data support the idea that DWPF's current viscosity model (using measured compositions) may underpredict the viscosity for uranium-bearing glasses in this compositional region. Specifically, the viscosity model may tend to overpredict the viscosity measurements for glasses without uranium but may tend to underpredict the viscosity for glasses with uranium in this compositional region. These results indicate that uranium may play a significant role in determining melt viscosity for glass compositions similar to those of DWPF's Macrobatch 2, as suggested in the interim report [3].

Alternate Salt Studies. A large number of glasses were fabricated for the alternative salt program in FY99 [6, 7]. The two options considered were (1) small tank - involving Precipitate Aqueous Hydrolysis (PHA) and (2) Crystalline Silicotitanate (CST) pretreatment. The glasses fabricated contained PHA from 7 to 13 weight percent (wt\%) or CST from 3 to 9 wt\%, with both sets of glasses containing approximately $2.0 \mathrm{wt} \%$ oxide depleted uranium. The melt viscosities of these glasses were determined by SRTC and the results were:

(1) The measured viscosities were generally higher than the predicted viscosities using both target and measured compositions and

(2) - The measured viscosities of the PHA glasses were closer to predicted values than was the case with CST glasses. 
These results do not appear to show the influence of uranium exhibited for the sludge-only glasses (Glasses 1, 3, and 5). Further work will be required to understand the influence of uranium on glass viscosity.

\section{CONCLUSIONS}

A Harrop, high-temperature glass viscometer has been installed, calibrated using the NIST 717a borosilicate glass standard, and tested at SRTC. This viscometer uses only 6 grams of glass per viscosity measurement. The repeatability of the instrument was checked over time using Batch 1 glass. The relative standard deviation of these data (expressed as a percentage) was $2.7 \%$.

The SRTC viscosity measurements at $1150^{\circ} \mathrm{C}$ (from the Fulcher fit of the data) for Batch 1, HM, and/or Purex glasses were compared with data from CELS, PNNL, and SS. The SRTC viscosity values were always higher than the CELS and SS values averaging about $9 \%$ with a range from 6 to $12 \%$. On the other hand the SRTC and PNNL results for the HM glass were different by only $0.2 \%$. Conducting these comparisons in (natural) log-space leads to even smaller differences. These results indicate that the SRTC Harrop Viscometer provides highly repeatable viscosity values at $1150^{\circ} \mathrm{C}$ and reasonable correspondence with CELS, PNNL, and SS data.

The 5 glasses fabricated to study the influence of uranium on viscosity were sent to PNNL (Glasses 1, 3, and 5) and to CELS (Glasses 2 and 4) for confirmation of the SRTC measurements. PNNL measured the three glasses containing uranium and the results were within $7 \%$ of the SRTC values. CELS measured the remaining two glasses that did not contain uranium. The results revealed that the SRTC viscosity values were $\sim 10 \%$ higher than those measured by CELS consistent with the Batch 1 results.

These five glasses are representative of DWPF's Macrobatch 2 (with and without uranium). The PNNL and SRTC data support the idea that DWPF's current viscosity model may underpredict the viscosity for uranium-bearing glasses in this compositional region. These results indicate that uranium may play a significant role in determining melt viscosity for glass compositions similar to those of DWPF's Macrobatch 2.

On the other hand, the results obtained with glasses produced through coupled flowsheets in an independent study for the Salt Alternatives Program do not appear to show the influence of uranium exhibited for the sludge-only glasses. Further work will be required to understand the influence of uranium on glass viscosity. 


\section{RECOMMENDATION}

Initiate a task to determine the influence of uranium on melt viscosity for sludgeonly glasses and, if necessary, incorporate a uranium term into the viscosity model for DWPF glasses.

\section{PATH FoRWARD}

In support of this recommendation, SRTC will seek funding to initiate a task to (A) develop a procedure for measurement of glass viscosity that identifies and minimizes the impact of volatility and crystallization on measured viscosities, (B) characterize the uncertainties in the measurements through a statistically designed set of experiments, and (C) provide the basis for an in-house capability for viscosity confirmation and model revision tasks.

Since this capability will benefit not only DWPF but also TFA, BNFL, and Salt Alternative programs joint funding will be sought.

\section{REFERENCES}

[1] Cicero-Herman, C. A., M. K. Andrews, and T. B. Edwards, "Results of the Tank 42 (Batch 1B) Variability Study," WSRC-TR-98-00180, June 5, 1998.

[2] Fellinger, T. L., et al., "Confirmation Run of the DWPF SME Cycle Using the SludgeOnly Flowsheet with Tank 42 Radioactive Sludge and Frit 200 in the Shielded Cells Facility (U)," WSRC-RP-98-00351, June 5, 1998.

[3] Schumacher, R. F., et al, "Measurement of DWPF Glass Viscosity-Interim Report (U), WSRC-RP-99-00350, Rev. 0, May 5, 1999.

[4] Schumacher, R. F. and D. K. Peeler, "Establishment of Harrop, High-Temperature Viscometer (U),"WSRC-RP-98-00737, Rev. 0, September, 1998.

[5] Brown, K. G. and R. L. Postles, "SME Acceptability Determination for DWPF Process Control (U), “WSRC-TR-95-0364, Revision 3, February 21, 1996.

[6] Edwards, T. B., J. R. Harbour, R. J. Workman, "Summary of Property Measurements from CST Glass Study (U)," WSRC-TR-99-00384, Rev. 0, October 4, 1999.

[7] Edwards, T. B., J. R. Harbour, R. J. Workman, "Summary of Results for PHA Glass Study: Composition and Property Measurements (U)," WSRC-TR-99-00332, Rev. 0, September 9, 1999. 
WSRC-RP-99-01053

Revision 0

\section{Appendix: Exhibits}


WSRC-RP-99-01053

Revision 0

This page intentionally left blank. 


\begin{tabular}{|c|c|c|c|c|c|c|}
\hline Date & $1 / 28 / 99$ & & & & & \\
\hline Glass & HM STD & & cruc.\&glass & 293.632 & & \\
\hline Goal & 6.638 & & crucible & 287.004 & & \\
\hline Actual amt & 6.628 & & glass & 6.628 & & \\
\hline TIME & TIME & SET RATE & FURNACE & SAMPLE & SPEED & $\%$ \\
\hline MIL & LEFT & ${ }^{\circ} \mathrm{C}$ & ${ }^{\circ} \mathrm{C}$ & ${ }^{\circ} \mathrm{C}$ & RPM & TORQUE \\
\hline 1135 & 0.13 & 1200 & 1200 & 1188 & 60 & 43.4 \\
\hline 1140 & 0.08 & 1200 & 1200 & 1188 & 60 & 43.4 \\
\hline 1145 & 0.03 & 1200 & 1200 & 1188 & 60 & 43.4 \\
\hline 1220 & 0.013 & 1150 & 1149 & 1135 & 30 & 33.6 \\
\hline 1225 & 0.08 & 1150 & 1150 & 1134 & 30 & 33.6 \\
\hline 1230 & 0.03 & 1150 & 1150 & 1134 & 30 & 33.6 \\
\hline 1310 & 0.08 & 1100 & 1100 & 1081 & 30 & 53.7 \\
\hline 1315 & 0.03 & 1100 & 1100 & 1081 & 30 & 53.7 \\
\hline 1320 & $.01 \mathrm{H}$ & 1100 & 1100 & 1081 & 30 & 53.7 \\
\hline 1400 & 0.08 & 1050 & 1049 & 1028 & 12 & 36.3 \\
\hline 1405 & 0.03 & 1050 & 1050 & 1028 & 12 & 36.3 \\
\hline 1410 & $.01 \mathrm{H}$ & 1050 & 1050 & 1028 & 12 & 36.3 \\
\hline 1445 & 0.11 & 1000 & 999 & 976 & 6 & 32.7 \\
\hline 1450 & 0.06 & 1000 & 999 & 976 & 6 & 32.6 \\
\hline 1455 & 0.01 & 1000 & 1000 & 976 & 6 & 32.5 \\
\hline & Sample & RPM & $\%$ Torque & $\mathrm{K}$ & Viscosity & \\
\hline & Ter & ure & & & Measured & \\
\hline & ${ }^{\circ} \mathrm{C}$ & & & & Poise & \\
\hline & 1188 & 60 & 43.4 & 123.01665 & 88.982042 & \\
\hline & 1134 & 60 & 33.6 & 126.44316 & 70.808172 & \\
\hline & 1081 & 30 & 53.7 & 129.80623 & 232.35314 & \\
\hline & 1028 & 12 & 36.3 & 133.16929 & 402.8371 & \\
\hline & 976 & 6 & 32.6 & 136.4689 & 741.481 & \\
\hline
\end{tabular}




\begin{tabular}{|c|c|c|c|c|c|c|}
\hline Date & $1 / 22 / 99$ & & & & & \\
\hline Glass & HM STD & & cruc.\&glass & 293.668 & & \\
\hline Goal & 6.6389 & & crucible & 287.036 & & \\
\hline Actual amt & 6.632 & & glass & 6.632 & & \\
\hline TIME & TIME & SET RATE & FURNACE & SAMPLE & SPEED & $\%$ \\
\hline MIL & LEFT & ${ }^{\circ} \mathbf{C}$ & ${ }^{\circ} \mathbf{C}$ & ${ }^{\circ} \mathbf{C}$ & RPM & TORQUE \\
\hline 1025 & 0.12 & 1200 & 1201 & 1187 & 30 & 21.7 \\
\hline 1030 & 0.07 & 1200 & 1200 & 1187 & 30 & 21.9 \\
\hline 1035 & 0.02 & 1200 & 1200 & 1187 & 30 & 21.9 \\
\hline 1040 & $.01 \mathrm{H}$ & 1200 & 1200 & 1187 & 60 & 43.7 \\
\hline 1045 & $.01 \mathrm{H}$ & 1200 & 1200 & 1187 & 60 & 43.7 \\
\hline 1123 & 0.1 & 1150 & 1149 & 1131 & 30 & 33.9 \\
\hline 1128 & 0.05 & 1150 & 1150 & 1131 & 30 & 33.9 \\
\hline 1133 & $.01 \mathrm{H}$ & 1150 & 1150 & 1131 & 30 & 33.9 \\
\hline 1205 & 0.12 & 1100 & 1099 & 1079 & 30 & 54.4 \\
\hline 1210 & 0.07 & 1100 & 1100 & 1079 & 30 & 54.3 \\
\hline 1215 & 0.02 & 1100 & 1100 & 1079 & 30 & 54.2 \\
\hline 1255 & 0.06 & 1050 & 1049 & 1027 & 12 & 36.7 \\
\hline 1300 & $.01 \mathrm{H}$ & 1050 & 1050 & 1027 & 12 & 36.6 \\
\hline 1305 & $.01 \mathrm{H}$ & 1050 & 1050 & 1027 & 12 & 36.6 \\
\hline 1240 & 0.1 & 1000 & 999 & 974 & 12 & 65.9 \\
\hline 1345 & 0.05 & 1000 & 1000 & 974 & 12 & 65.7 \\
\hline \multirow[t]{9}{*}{1350} & $.01 \mathrm{H}$ & 1000 & 1000 & 974 & 12 & 65.7 \\
\hline & Sample & RPM & $\%$ Torque & $\mathrm{K}$ & Viscosity & \\
\hline & \multicolumn{2}{|c|}{ Temperature } & & & Measured & \\
\hline & ${ }^{\circ} \mathrm{C}$ & & & & Poise & \\
\hline & 1187 & 60 & 43.7 & 123.0801 & 89.643341 & \\
\hline & 1131 & 30 & 33.9 & 126.63353 & 143.09588 & \\
\hline & 1079 & 30 & 54.3 & 129.93313 & 235.17897 & \\
\hline & 1027 & 12 & 36.65 & 133.23274 & 406.915 & \\
\hline & 974 & 12 & 65.75 & 136.5958 & 748.43118 & \\
\hline
\end{tabular}




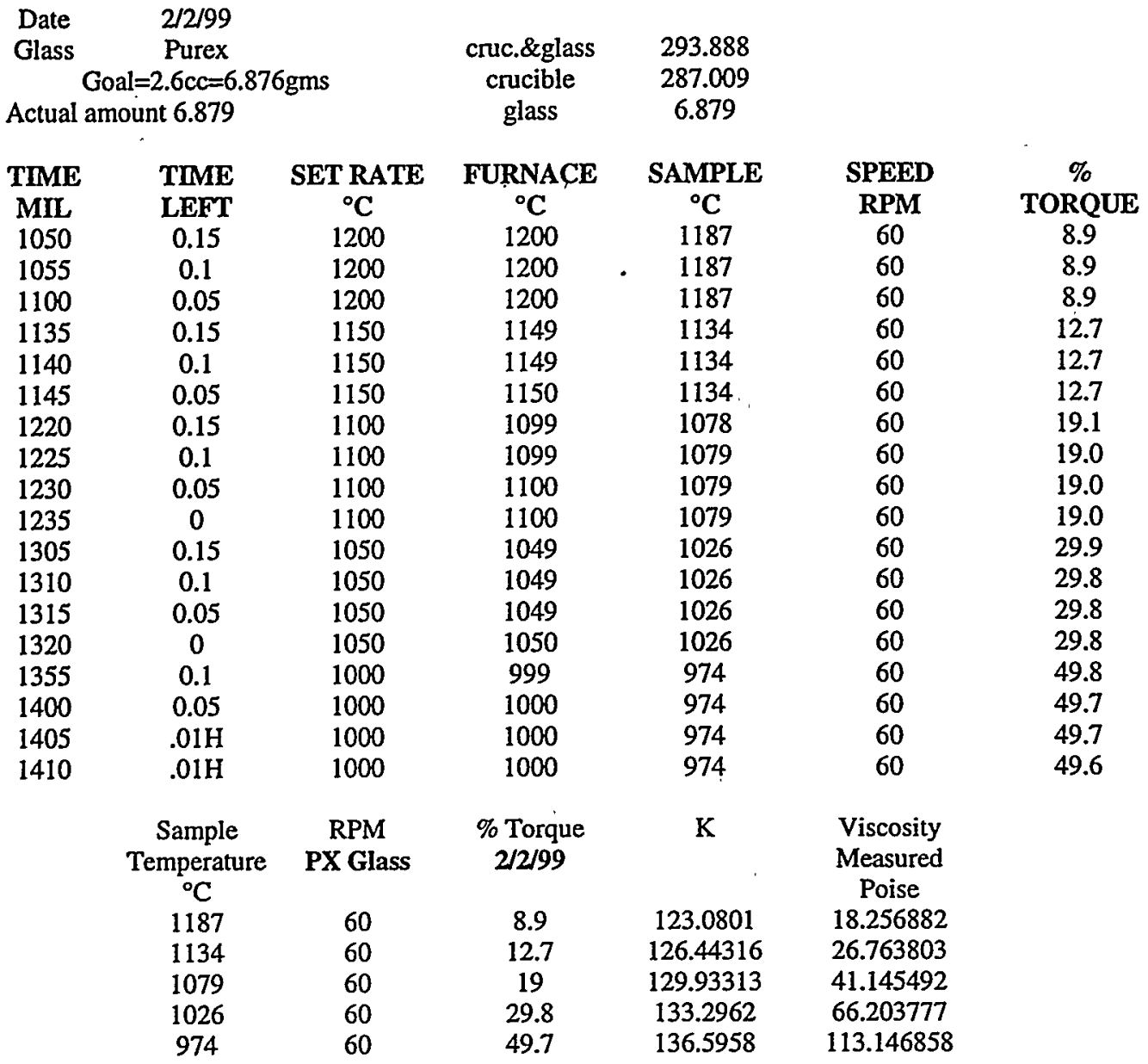




\begin{tabular}{|c|c|c|c|c|c|c|}
\hline Date & $2 / 5 / 99$ & & & & & \\
\hline Glass & Purex & & cruc.\&glass & 293.945 & & \\
\hline Goal 2. & $=6.876 \mathrm{gms}$ & & crucible & 287.066 & & \\
\hline Actual & lount 6.879 & & glass & 6.879 & & \\
\hline TIME & TMME & SET RATE & FURNACE & SAMPLE & SPEED & $\%$ \\
\hline MUL & LEFT & ${ }^{\circ} \mathrm{C}$ & ${ }^{\circ} \mathrm{C}$ & ${ }^{\circ} \mathrm{C}$ & RPM & TORQUE \\
\hline 950 & 0.12 & 1200 & 1200 & 1187 & 60 & 8.45 \\
\hline 955 & 0.07 & 1200 & 1200 & 1187 & 60 & 8.45 \\
\hline 960 & 0.02 & 1200 & 1200 & 1187 & 60 & 8.45 \\
\hline 1040 & 0.07 & 1150 & 1150 & 1134 & 60 & 12 \\
\hline 1045 & 0.02 & 1150 & 1150 & 1132 & 60 & 12.1 \\
\hline 1050 & $0.01 \mathrm{H}$ & 1150 & 1150 & 1133 & 60 & 12.05 \\
\hline 1122 & 0.12 & 1100 & 1099 & 1079 & 60 & 18.05 \\
\hline 1127 & 0.07 & 1100 & 1099 & 1079 & 60 & 18 \\
\hline 1132 & 0.02 & 1100 & 1100 & 1079 & 60 & 18 \\
\hline 1205 & 0.13 & 1050 & 1049 & 1026 & 60 & 28.4 \\
\hline 1210 & 0.08 & 1050 & 1049 & 1026 & 60 & 28.3 \\
\hline 1215 & 0.03 & 1050 & 1049 & 1026 & 60 & 28.2 \\
\hline 1250 & 0.14 & 1000 & 999 & 974 & 60 & 47.05 \\
\hline 1255 & 0.09 & 1000 & 999 & 974 & 60 & 46.8 \\
\hline 1300 & 0.04 & 1000 & 999 & 974 & 60 & 46.6 \\
\hline 1305 & $0.01 \mathrm{H}$ & 1000 & 1000 & 974 & 60 & 46.6 \\
\hline & Sample & RPM & $\%$ Torque & K & Viscosity & \\
\hline & $\begin{array}{c}\text { Temperature } \\
{ }^{\circ} \mathrm{C}\end{array}$ & PX Glass & 2/5/99 & & $\begin{array}{c}\text { Measured } \\
\text { Poise }\end{array}$ & \\
\hline & 1187 & 60 & 8.45 & 123.0801 & 17.333781 & \\
\hline & 1133 & 60 & 12.05 & 126.50662 & 25.406746 & \\
\hline & 1079 & 60 & 18 & 129.93313 & 38.97994 & \\
\hline & 1026 & 60 & 28.3 & 133.2962 & 62.871372 & \\
\hline & 974 & 60 & 46.67 & 136.5958 & 106.24877 & \\
\hline
\end{tabular}




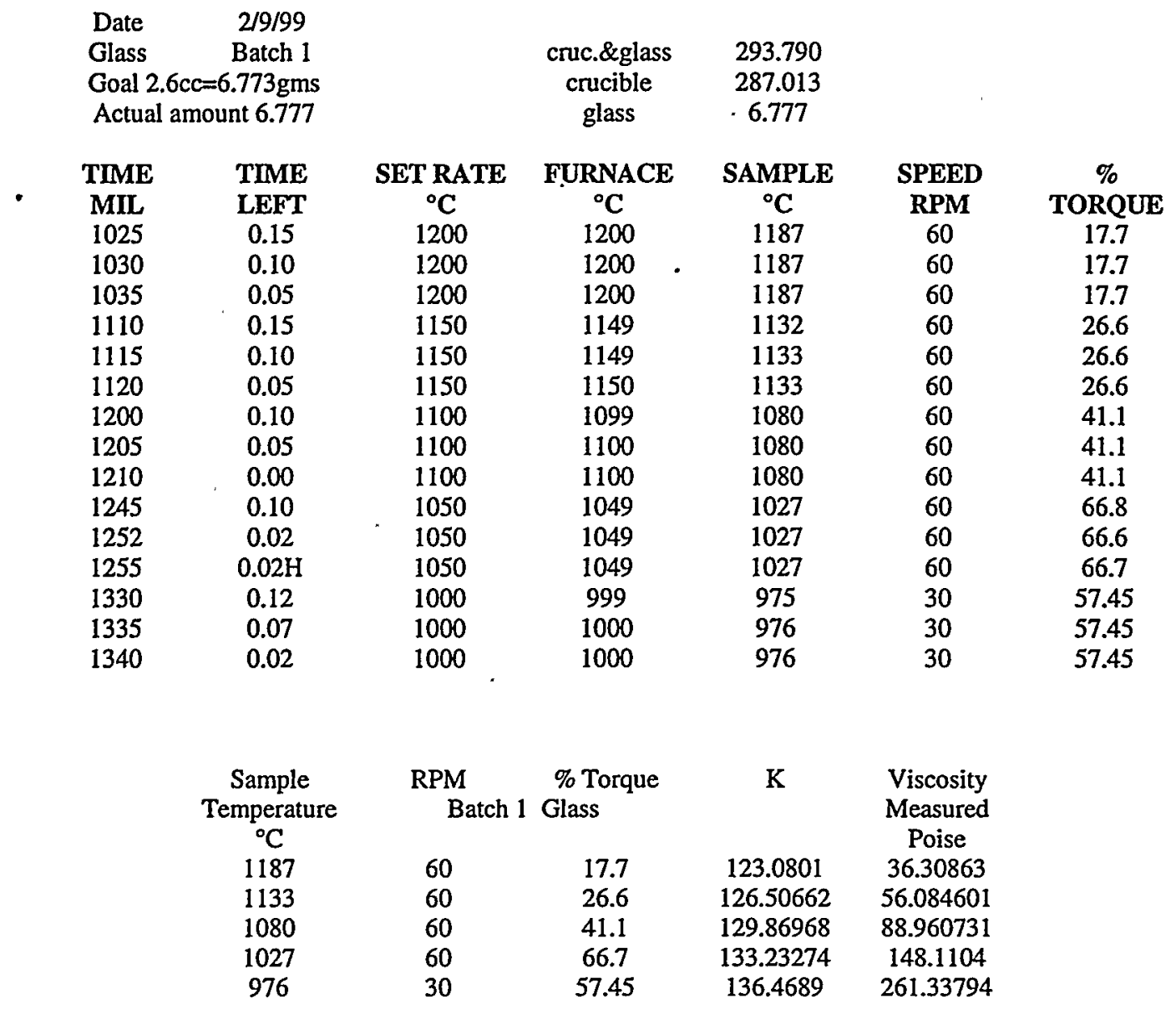




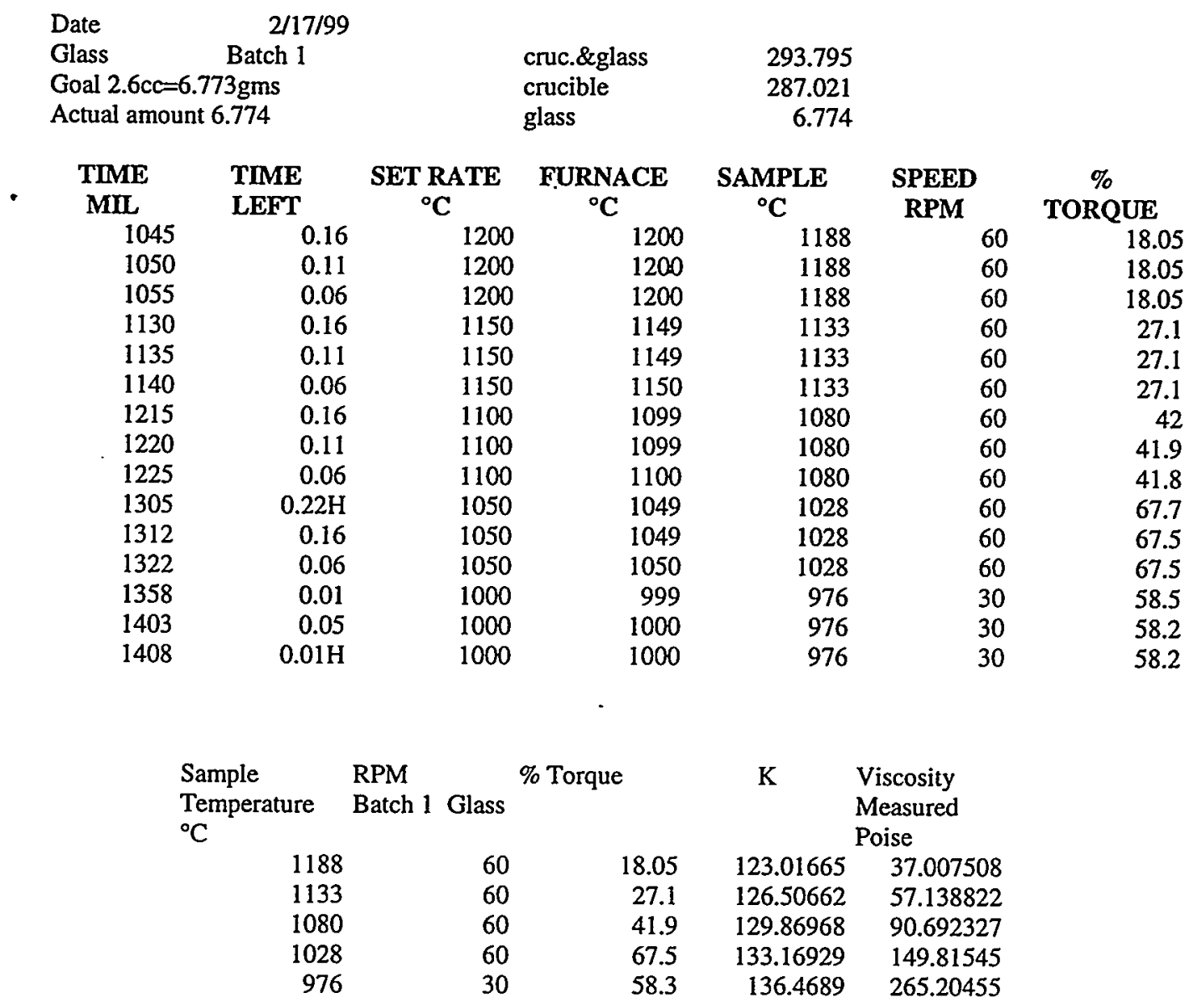




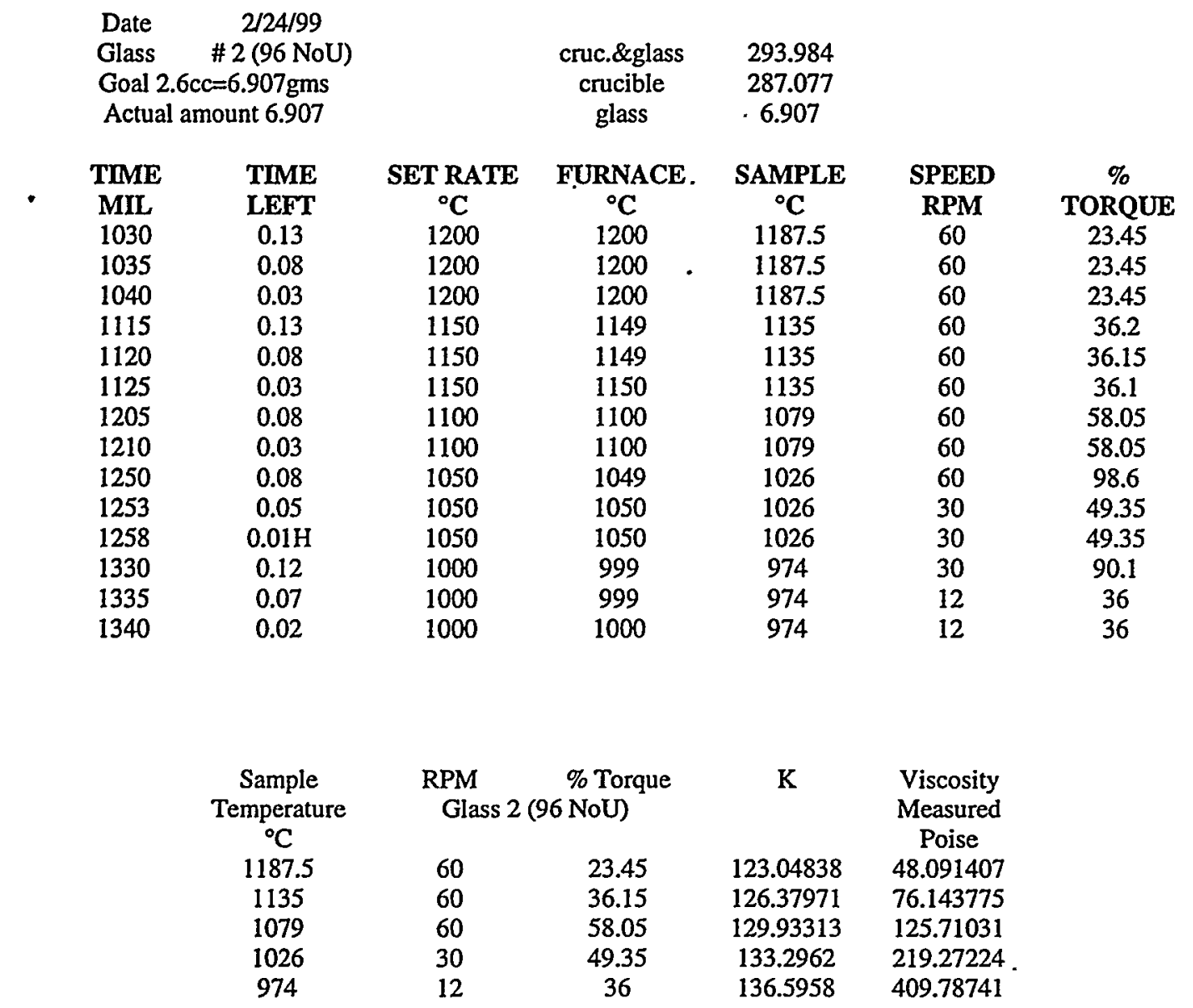




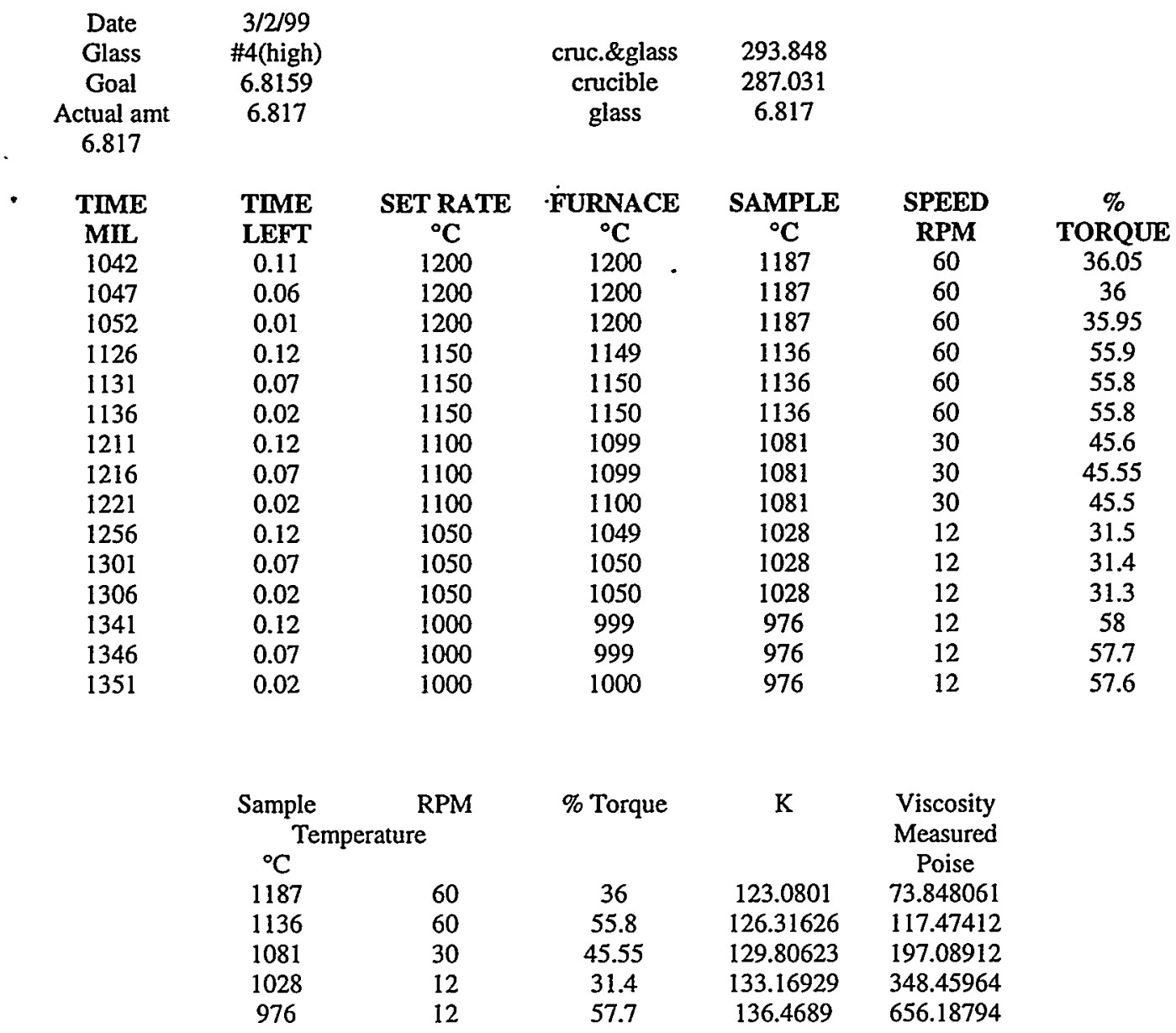


Appendix: Exhibits

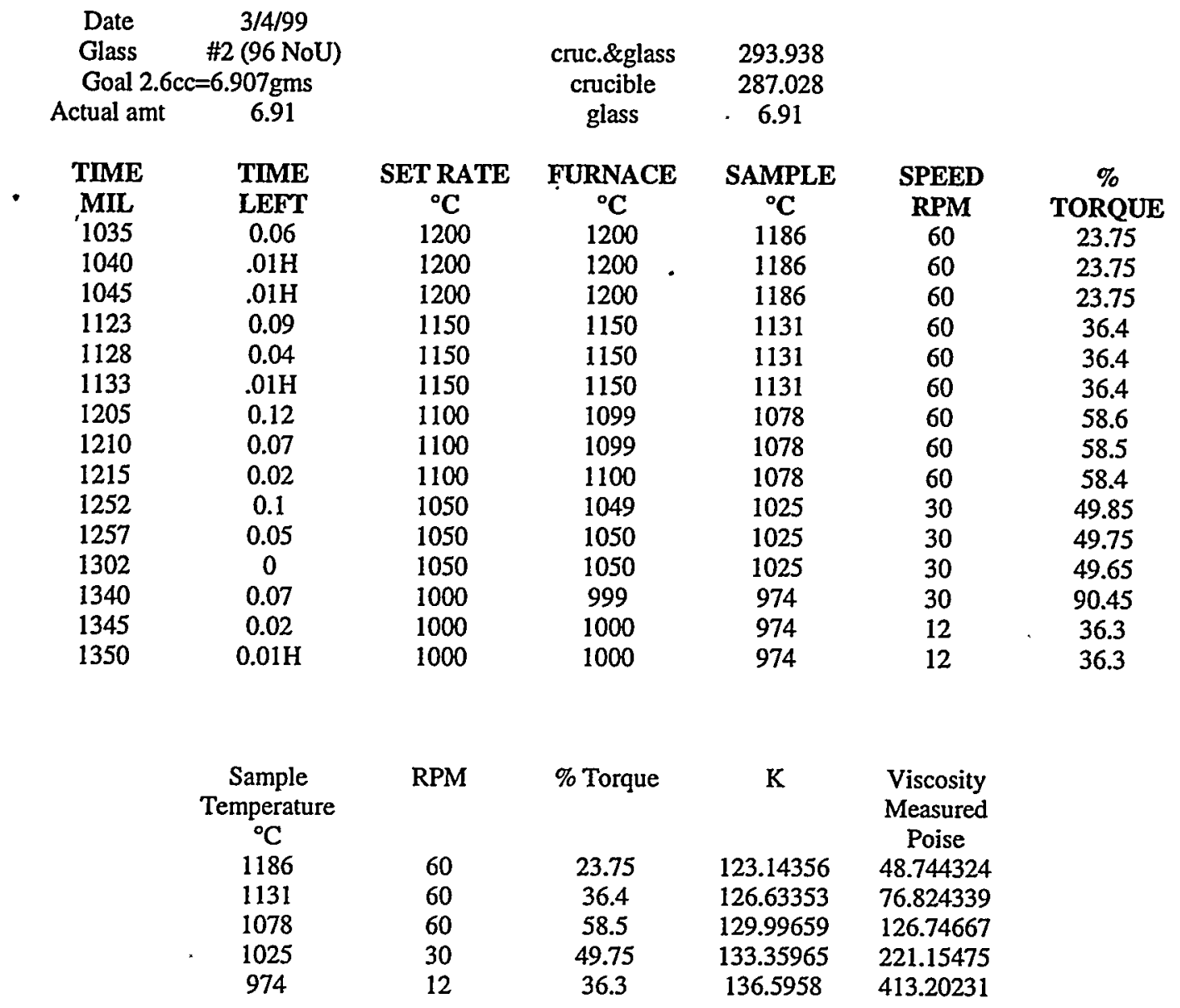




\begin{tabular}{|c|c|c|c|c|c|c|}
\hline Date & $3 / 9 / 99$ & & & & & \\
\hline Glass & \#4(high) & & cruc.\&glass & 293.887 & & \\
\hline Goal & 6.8159 & & crucible & 287.067 & & \\
\hline Actual amt & 6.82 & $\cdot$ & glass & 6.82 & & \\
\hline TIME & TIME & SET RATE & FURNACE & SAMPLE & SPEED & $\%$ \\
\hline MLL & LEFT & ${ }^{\circ} \mathrm{C}$ & ${ }^{\circ} \mathrm{C}$ & ${ }^{\circ} \mathrm{C}$ & RPM & TORQUE \\
\hline 1010 & 0.1 & 1200 & 1200 & 1188 & 60 & 36.35 \\
\hline 1015 & 0.05 & 1200 & 1200 & 1188 & 60 & 36.35 \\
\hline 1020 & 0 & 1200 & 1200 & 1188 & 60 & 36.35 \\
\hline 1055 & 0.1 & 1150 & 1150 & 1132 & 60 & 56.25 \\
\hline 1100 & 0.05 & 1150 & 1150 & 1133 & 60 & 56.2 \\
\hline 1105 & 0 & 1150 & 1150 & 1133 & 60 & 56.2 \\
\hline 1140 & 0.1 & 1100 & 1099 & 1080 & 30 & 45.95 \\
\hline 1145 & 0.05 & 1100 & 1100 & 1080 & 30 & 45.95 \\
\hline 1150 & 0 & 1100 & 1100 & 1080 & 30 & 45.95 \\
\hline 1310 & 0.1 & 1000 & 999 & 974 & 12 & 58 \\
\hline 1315 & 0.05 & 1000 & 1000 & 975 & 12 & 57.8 \\
\hline 1320 & 0 & 1000 & 1000 & 975 & 12 & 57.8 \\
\hline & Sample & RPM & $\%$ Torque & $\mathrm{K}$ & \multicolumn{2}{|l|}{ Viscosity } \\
\hline \multicolumn{5}{|c|}{ Temperature } & \multirow{2}{*}{\multicolumn{2}{|c|}{$\begin{array}{c}\text { Measured } \\
\text { Poise }\end{array}$}} \\
\hline & ${ }^{\circ} \mathrm{C}$ & & & & & \\
\hline & 1188 & 60 & 36.35 & 123.01665 & 74.527586 & \\
\hline & 1133 & 60 & 56.2 & 126.50662 & 118.49453 & \\
\hline & 1080 & 30 & 45.95 & 129.86968 & 198.91706 & \\
\hline & 975 & 12 & 57.8 & 136.53235 & 657.63082 & \\
\hline
\end{tabular}




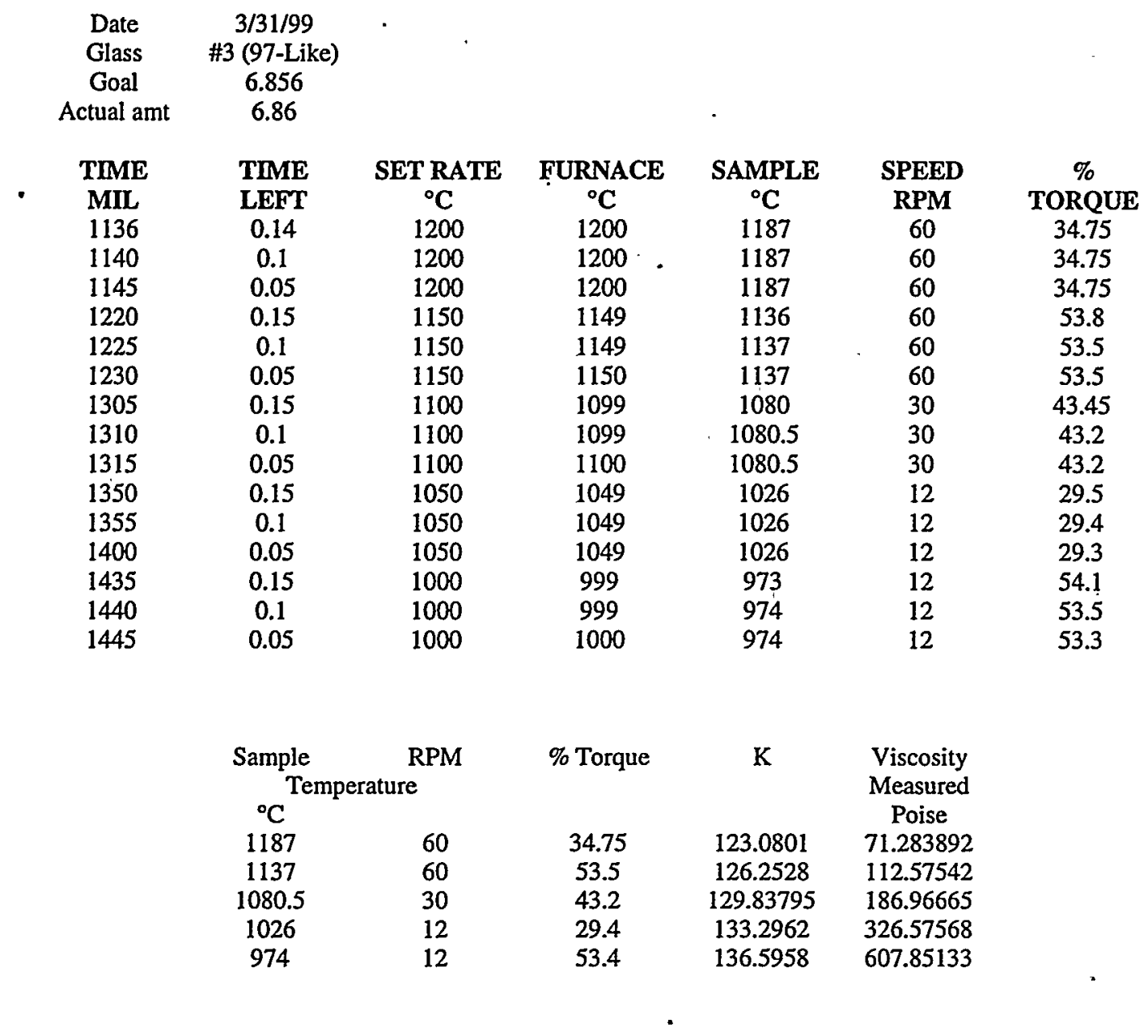




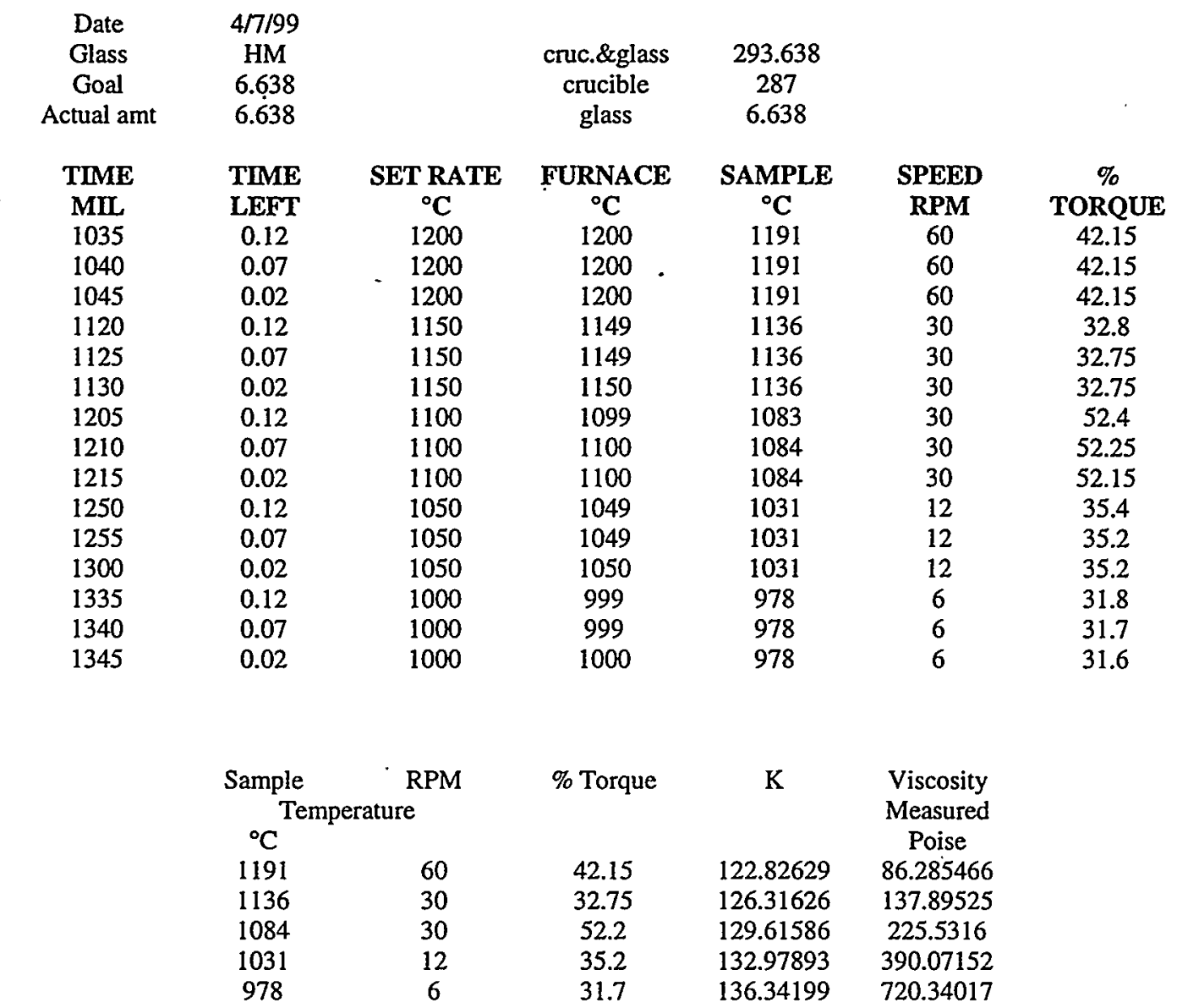




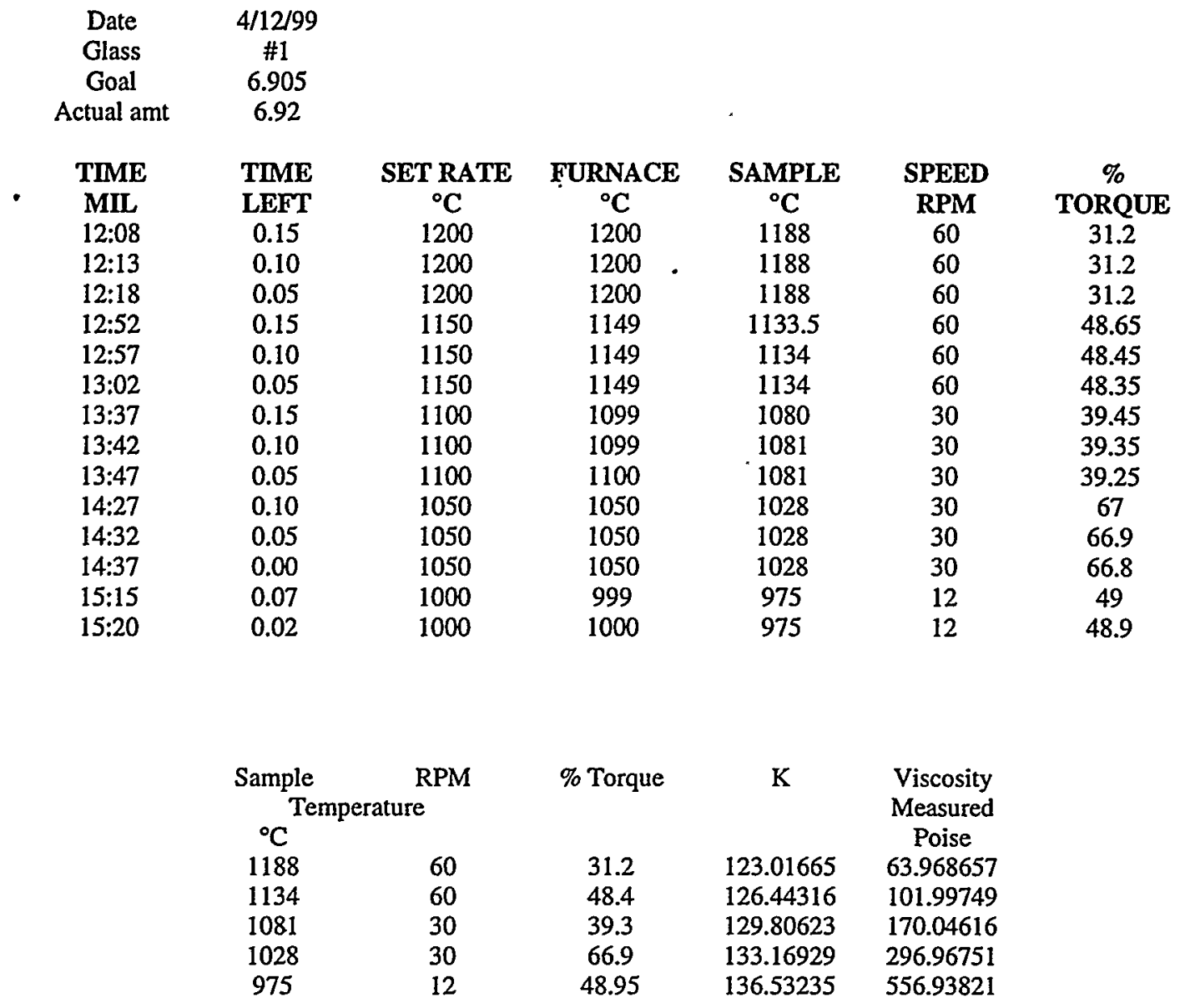




\begin{tabular}{|c|c|c|c|c|c|c|}
\hline Date & $4 / 15 / 99$ & & & & & \\
\hline Glass & \#5 & & & & & \\
\hline Goal & 6.852 & & & & & \\
\hline Actual amt & 6.852 & & & & & \\
\hline - TIME & TIME & SET RATE & FURNACE & SAMPLE & SPEED & $\%$ \\
\hline MIL & LEFT & ${ }^{\circ} \mathrm{C}$ & ${ }^{\circ} \mathrm{C}$ & ${ }^{\circ} \mathrm{C}$ & RPM & TORQUE \\
\hline 1027 & 0.15 & 1200 & 1200 & - 1188 & 60 & 37.75 \\
\hline 1032 & 0.1 & 1200 & 1200 & 1188 & 60 & 37.8 \\
\hline 1037 & 0.05 & 1200 & 1200 & 1188 & 60 & 37.8 \\
\hline 1114 & 0.12 & 1150 & 1149 & 1135.5 & 60 & 58.8 \\
\hline 1119 & 0.07 & 1150 & 1150 & 1135.5 & 60 & 58.7 \\
\hline 1124 & 0.02 & 1150 & 1150 & 1135.5 & 60 & 58.6 \\
\hline 1157 & 0.15 & 1100 & 1099 & 1079.5 & 30 & 47.75 \\
\hline 1202 & 0.1 & 1100 & 1099 & 1080 & 30 & 47.6 \\
\hline 1207 & 0.05 & 1100 & 1100 & 1080 & 30 & 47.4 \\
\hline 1242 & 0.15 & 1050 & 1049 & 1026.5 & 12 & 32.8 \\
\hline 1247 & 0.1 & 1050 & 1049 & 1027 & 12 & 32.75 \\
\hline 1252 & 0.05 & 1050 & 1049 & 1027 & 12 & 32.65 \\
\hline 1327 & 0.15 & 1000 & 999 & 974 & 12 & 60 \\
\hline 1332 & 0.1 & 1000 & 999 & 975.5 & 12 & 59.3 \\
\hline 1337 & 0.05 & 1000 & 999 & 975 & 12 & 59.1 \\
\hline
\end{tabular}

$\begin{array}{ccccc}\begin{array}{c}\text { Sample } \\ \text { Temperature }\end{array} & \text { RPM } & \text { \% Torque } & \text { K } & \begin{array}{c}\text { Viscosity } \\ \text { Measured } \\ \text { Poise }\end{array} \\ { }^{\circ} \mathrm{C} & & & & 77.500488 \\ 1188 & 60 & 37.8 & 123.01665 & 72.61044 \\ 1135.5 & 60 & 58.7 & 126.34798 & 123.610459 \\ 1080 & 30 & 47.5 & 129.86968 & 205.62699 \\ 1027 & 12 & 32.7 & 133.23274 & 363.05922 \\ 975 & 12 & 59.4 & 136.53235 & 675.83513\end{array}$


Appendix: Exhibits

WSRC-RP-99-01053

Exhibit A.1: SRTC Viscometer Results in Date Order

Revision 0

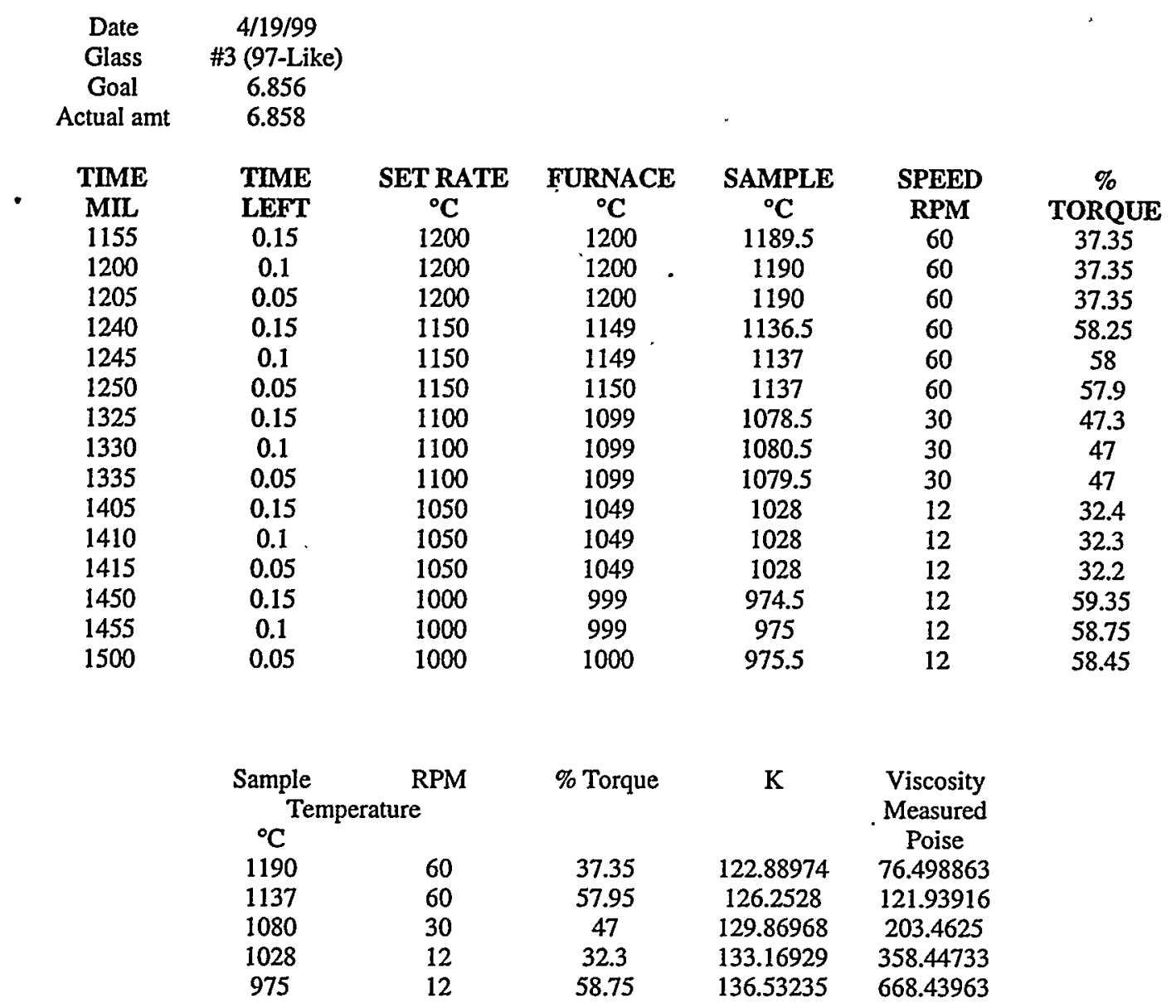




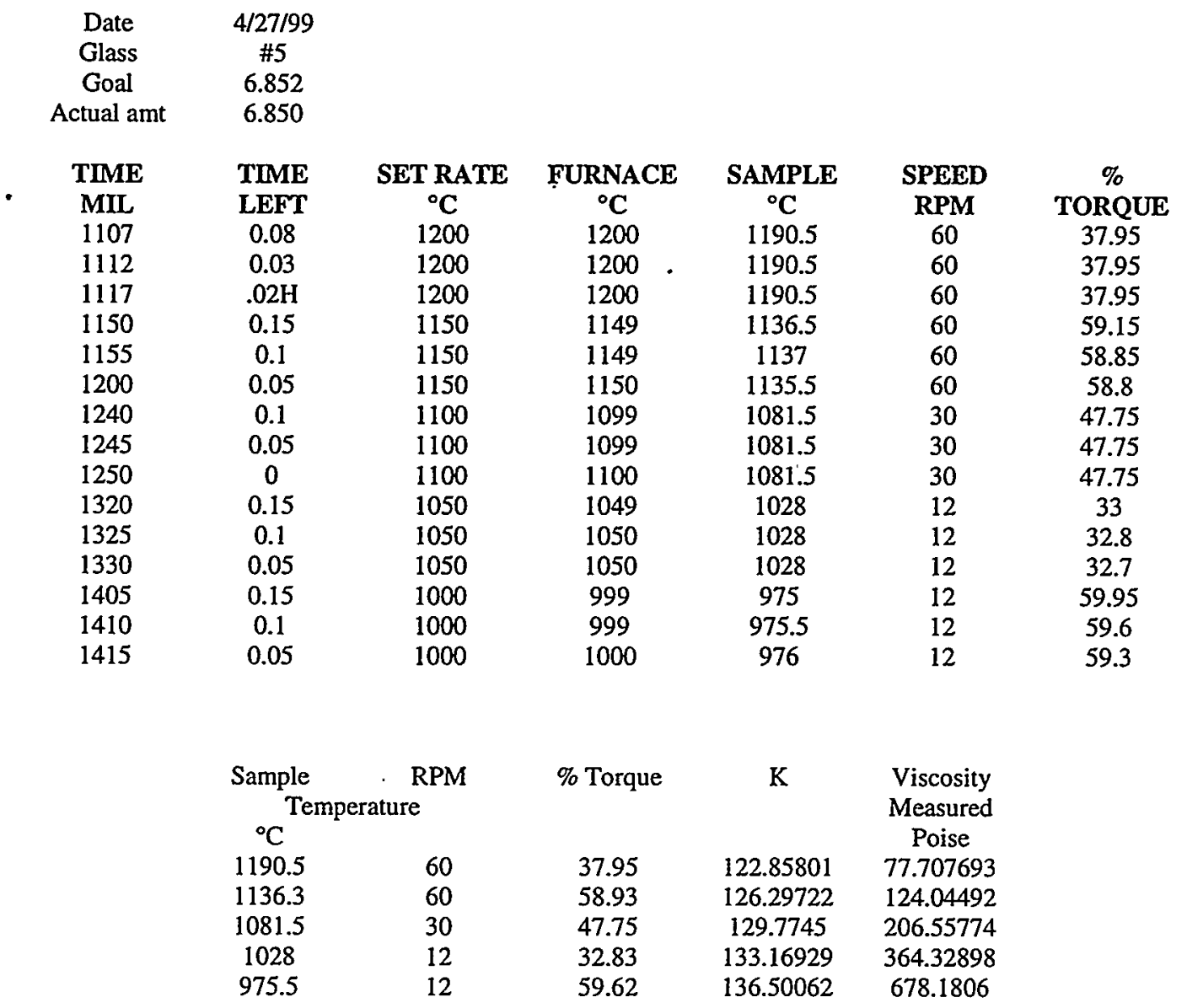




\begin{tabular}{|c|c|c|c|c|c|c|}
\hline Date & $4 / 28 / 99$ & & NEW CR & CIBLE AND & PINDLE & \\
\hline Glass & HM & & cruc.\&glass & 291.516 & & \\
\hline Goal & 6.638 & & crucible & 284.875 & & \\
\hline Actual amt & 6.641 & & glass & 6.641 & & \\
\hline TIME & TIME & SET RATE & FUURACE & SAMPLE & SPEED & $\%$ \\
\hline MIL & LEFT & ${ }^{\circ} \mathrm{C}$ & ${ }^{\circ} \mathrm{C}$ & ${ }^{\circ} \mathrm{C}$ & RPM & TORQUE \\
\hline 1205 & $0.05 \mathrm{H}$ & 1200 & 1200 & 1193 & 60 & 41.25 \\
\hline 1210 & $0.05 \mathrm{H}$ & 1200 & 1200 & 1193 & 60 & 41.25 \\
\hline 1215 & 0.04 & 1200 & 1200 & 1193 & 60 & 41.35 \\
\hline 1250 & 0.14 & 1150 & 1149 & 1139 & 60 & 63.8 \\
\hline 1255 & 0.09 & 1150 & 1149 & 1138.5 & 60 & 63.65 \\
\hline 1300 & 0.04 & 1150 & 1150 & 1138.5 & 60 & 63.65 \\
\hline 1340 & 0.09 & 1100 & 1099 & 1083.5 & 30 & 51.1 \\
\hline 1345 & 0.04 & 1100 & 1100 & 1083.5 & 30 & 51.05 \\
\hline 1350 & $0.01 \mathrm{H}$ & 1100 & 1100 & 1083.5 & 30 & 51 \\
\hline 1424 & 0.1 & 1050 & 1049 & 1030.5 & 30 & 85.95 \\
\hline 1429 & 0.05 & 1050 & 1050 & 1030.5 & 30 & 85.7 \\
\hline 1434 & 0 & 1050 & 1050 & 1030.5 & 30 & 85.7 \\
\hline 1505 & 0.15 & 1000 & 999 & 977 & 12 & 62.3 \\
\hline 1510 & 0.1 & 1000 & 999 & 977.5 & 12 & 61.8 \\
\hline 1515 & 0.05 & 1000 & 1000 & 977.5 & 12 & 61.6 \\
\hline & & & & & & \\
\hline & Sample & RPM & $\%$ Torque & K & Viscosity & \\
\hline & $\mathrm{Te}$ & cature & & & Measured & \\
\hline & ${ }^{\circ} \mathrm{C}$ & & & & Poise & \\
\hline & 1193 & 60 & 41.3 & 122.69938 & 84.458072 & \\
\hline & 1138.5 & 60 & 63.65 & 126.15762 & 133.83221 & \\
\hline & 1083.5 & 30 & 51.05 & 129.64759 & 220.61698 & \\
\hline & 1030.5 & 30 & 85.78 & 133.01065 & 380.32179 & \\
\hline & 977.5 & 12 & 61.7 & 136.37372 & 701.18818 & \\
\hline
\end{tabular}




\begin{tabular}{|c|c|c|c|c|c|c|}
\hline Date & $4 / 30 / 99$ & & \multicolumn{2}{|c|}{ Crucible B and Spindle B } & & \\
\hline Glass & Batch 1 & & cruc.\&glass & 291.683 & & \\
\hline Goal & 6.773 & & crucible & 284.905 & & \\
\hline Actual amt & 6.778 & & glass & 6.778 & & \\
\hline TIME & TIME & SET RATE & FURNACE & SAMPLE & SPEED & $\%$ \\
\hline MIL & LEFT & ${ }^{\circ} \mathrm{C}$ & ${ }^{\circ} \mathrm{C}$ & ${ }^{\circ} \mathrm{C}$ & RPM & TORQUE \\
\hline 1033 & 0.15 & 1200 & 1200 & 1194.5 & 60 & 16.2 \\
\hline 1038 & 0.1 & 1200 & 1200 & 1194 & 60 & 16.2 \\
\hline 1043 & 0.05 & 1200 & 1200 & 1194 & 60 & 16.2 \\
\hline 1118 & 0.15 & 1150 & 1149 & 1137 & 60 & 24.2 \\
\hline 1123 & 0.1 & 1150 & 1150 & 1138 & 60 & 24 \\
\hline 1128 & 0.05 & 1150 & 1150 & 1141 & 60 & 24 \\
\hline 1203 & 0.15 & 1100 & 1099 & 1085.5 & 60 & 37.3 \\
\hline 1208 & 0.1 & 1100 & 1100 & 1086 & 60 & 37.2 \\
\hline 1213 & 0.05 & 1100 & 1100 & 1086 & 60 & 37.2 \\
\hline 1248 & 0.15 & 1050 & 1049 & 1031.5 & 60 & 60.55 \\
\hline 1253 & 0.1 & 1050 & 1049 & 1032 & 60 & 60.2 \\
\hline 1258 & 0.05 & 1050 & 1050 & 1032 & 60 & 60.1 \\
\hline 1333 & 0.15 & 1000 & 999 & 978.5 & 30 & 52.1 \\
\hline 1338 & 0.1 & 1000 & 999 & 979 & 30 & 51.8 \\
\hline 1343 & 0.05 & 1000 & 1000 & 979 & 30 & 51.6 \\
\hline & Sample & RPM & $\%$ Torque & $\mathrm{K}$ & Viscosity & \\
\hline & $\mathrm{Ter}$ & are & & & Measured & \\
\hline & ${ }^{\circ} \mathrm{C}$ & & & & Poise & \\
\hline & 1194 & 60 & 16.2 & 122.63592 & 33.111699 & \\
\hline & 1139 & 60 & 24 & 126.12589 & 50.450358 & \\
\hline & 1086 & 60 & 37.2 & 129.48896 & 80.283153 & \\
\hline & 1032 & 60 & 60.15 & 132.91547 & 133.24776 & \\
\hline & 979 & 30 & 51.7 & 136.27853 & 234.85334 & \\
\hline
\end{tabular}




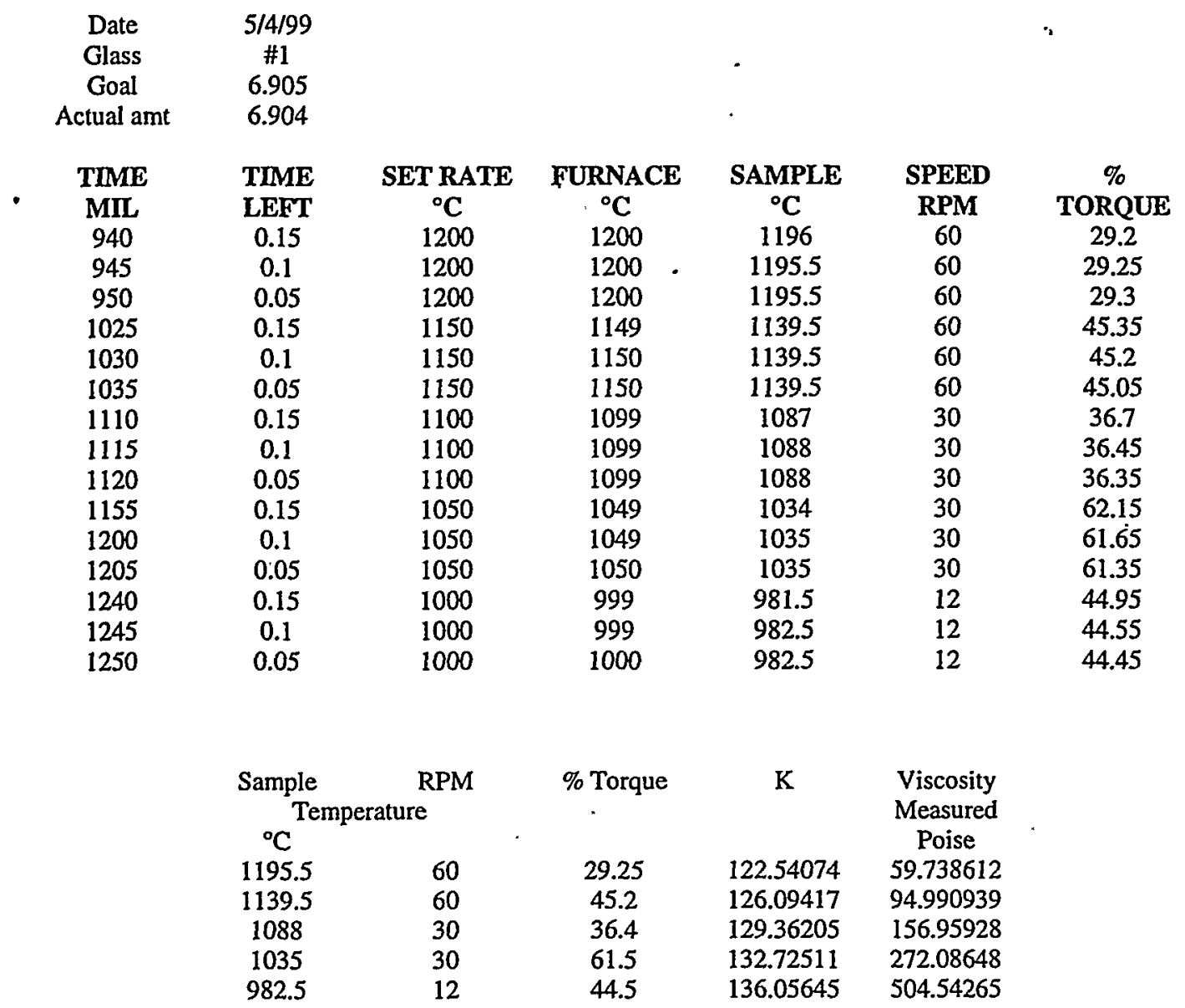




\begin{tabular}{|c|c|c|c|c|c|c|}
\hline Date & $5 / 7 / 99$ & & \multicolumn{2}{|c|}{ Crucible B and Spindle B } & & \\
\hline Glass & Purex & & cruc.\&glass & 291.749 & & \\
\hline Goal & 6.876 & & crucible & 284.872 & & \\
\hline Actual amt & 6.877 & & glass & 6.877 & & \\
\hline TIME & TIME & SET RATE & FURNACE & SAMPLE & SPEED & $\%$ \\
\hline MLL & LEFT & ${ }^{\circ} \mathbf{C}$ & ${ }^{\circ} \mathrm{C}$ & ${ }^{\circ} \mathrm{C}$ & RPM & TORQUE \\
\hline 1025 & 0.1 & 1200 & 1200 & 1196.5 & 60 & 7.55 \\
\hline 1030 & 0.05 & 1200 & 1200 & 1196.5 & 60 & 7.55 \\
\hline 1035 & 0 & 1200 & 1200 & 1196.5 & 60 & 7.55 \\
\hline 1110 & 0.1 & 1150 & 1150 & 1141.5 & 60 & 10.85 \\
\hline 1115 & 0.05 & 1150 & 1150 & 1141.5 & 60 & 10.85 \\
\hline 1120 & 0 & 1150 & 1150 & 1141.5 & 60 & 10.85 \\
\hline 1155 & 0.1 & 1100 & 1099 & 1088 & 60 & 16.2 \\
\hline 1200 & 0.05 & 1100 & 1100 & 1088 & 60 & 16.2 \\
\hline 1205 & 0 & 1100 & 1100 & 1088 & 60 & 16.2 \\
\hline 1240 & 0.1 & 1050 & 1049 & 1035 & 60 & 25.25 \\
\hline 1245 & 0.05 & 1050 & 1049 & 1035 & 60 & 25.2 \\
\hline 1250 & 0 & 1050 & 1050 & 1035 & 60 & 25.15 \\
\hline 1325 & 0.1 & 1000 & 999 & 982.5 & 60. & 41.85 \\
\hline 1330 & 0.05 & 1000 & 999 & 982.5 & 60 & 41.6 \\
\hline 1335 & 0 & 1000 & 1000 & 982.5 & 60 & 41.55 \\
\hline & Sample & RPM & \% Torque & K & Viscosity & \\
\hline & & ature & & & Measured & \\
\hline & ${ }^{\circ} \mathrm{C}$ & & & & Poise & \\
\hline & 1196.5 & 60 & 7.55 & 122.47729 & 15.411726 & \\
\hline & 1141.5 & 60 & 10.85 & 125.96726 & 22.779079 & \\
\hline & 1088 & 60 & 16.2 & 129.36205 & 34.927753 & \\
\hline & 1035 & 60 & 25.2 & 132.72511 & 55.744546 & \\
\hline & 982.5 & 60 & 41.65 & 136.05645 & 94.445849 & \\
\hline
\end{tabular}




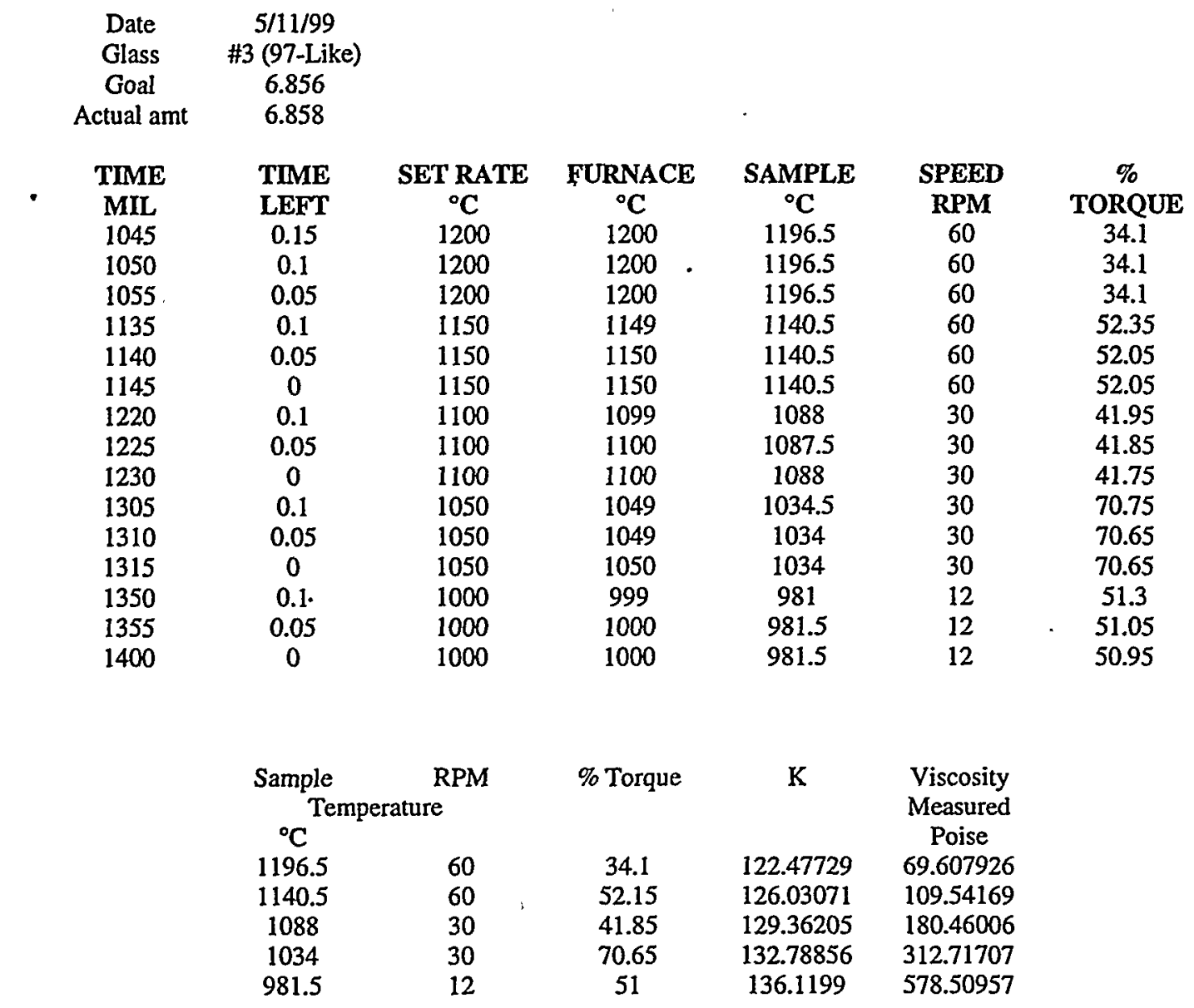


$\begin{array}{lc}\text { Date } & 6 / 8 / 99 \\ \text { Glass } & \text { Batch 1 } \\ \text { Goal 2.6cc }=6.773 \mathrm{gms}\end{array}$

Actual amount 6.780

TIME TIME

MIL LEFT

$1209 \quad 0.10$

$1214 \quad 0.05$

$1219 \quad 0.00$

$1254 \quad 0.10$

1259

1304

1339

1344

1349

1424

1429

1434

1509

1415

1519
0.10
0.05

0.00

0.10

0.05

0.00

0.10

0.05

0.00

0.10

0.05

0.00

Sample

Temperature

$$
{ }^{\circ} \mathrm{C}
$$

1199.5

1089.5

1037

984

$\begin{array}{cc}\text { SET RATE } & \begin{array}{c}\text { Crucible } \\ \text { cruc.\&glass } \\ \text { crucible } \\ \text { glass }\end{array} \\ \begin{array}{c}{ }^{\circ} \mathrm{C} \\ 1200\end{array} & \begin{array}{c}{ }^{\circ} \mathrm{C} \\ 1200\end{array} \\ 1200 & 1200 \\ 1200 & 1200 \\ 1150 & 1200 \\ 1150 & 1149 \\ 1150 & 1150 \\ 1100 & 1150 \\ 1100 & 1099 \\ 1100 & 1100 \\ 1050 & 1100 \\ 1050 & 1049 \\ 1050 & 1049 \\ 1000 & 1050 \\ 1000 & 999 \\ 1000 & 1000 \\ & 1000\end{array}$

Cruc.\&glass

Spindle A

293.800

287.020

6.780

\section{RNAC \\ SAMPLE \\ ${ }^{\circ} \mathrm{C}$}

1199.5

1199.5

1199.5

1143.5

1143.5

1143.5

1089.5

1089.5

1089.5

1037

1037

1037

983.5

984

984

SPEED
RPM
60
60
60
60
60
60
60
60
60
60
60
60
30
30
30

$\%$

TORQUE

16.5

16.5

16.5

24.55

24.55

24.55

37.8

37.75

37.75

61.1

61

60.9

52.35

52.2

52.2 


\begin{tabular}{|c|c|c|c|c|c|c|}
\hline $\begin{array}{l}\text { Date } \\
\text { Glass } \\
\text { Goal 2.6cc=6 } \\
\text { Actual amou }\end{array}$ & $\begin{array}{l}\quad 6 / 9 / 99 \\
\text { Batch } 1 \\
\text { 3gms } \\
.772\end{array}$ & & $\begin{array}{l}\text { Crucible \& Spin } \\
\text { cruc.\&glass } \\
\text { crucible } \\
\text { glass }\end{array}$ & $\begin{array}{lr}\text { He B } \\
\\
291.672 \\
284.900 \\
. \quad 6.772\end{array}$ & & \\
\hline TIME & TIME & SET RATE & FURNACE & SAMPLE & SPEED & $\%$ \\
\hline MIL & LEFT & ${ }^{\circ} \mathrm{C}$ & ${ }^{\circ} \mathrm{C}$ & ${ }^{\circ} \mathrm{C}$ & RPM & TORQUE \\
\hline 925 & 0.10 & 1200 & 1200 & 1197.5 & 60 & 16.3 \\
\hline 930 & 0.05 & 1200 & 1200 & 1198 & 60. & 16.3 \\
\hline 935 & 0.00 & 1200 & 1200 & 1198.5 & 60 & 16.3 \\
\hline 1010 & 0.10 & 1150 & 1149 & 1141.5 & 60 & 23.85 \\
\hline 1015 & 0.05 & 1150 & 1150 & 1142 & 60 & 23.85 \\
\hline 1020 & 0.00 & 1150 & 1150 & 1142 & 60 & 23.85 \\
\hline 1055 & 0.10 & 1100 & 1099 & 1088.5 & 60 & 36.45 \\
\hline 1100 & 0.05 & 1100 & 1100 & 1089 & 60 & 36.45 \\
\hline 1105 & 0.00 & 1100 & 1100 & 1089 & 60 & 36.45 \\
\hline 1140 & 0.10 & 1050 & 1049 & 1035.5 & 60 & 59.05 \\
\hline 1145 & 0.05 & 1050 & 1050 & 1035.5 & 60 & 59.05 \\
\hline 1150 & 0.00 & 1050 & 1050 & 1035.5 & 60 & 59.05 \\
\hline 1225 & 0.10 & 1000 & 999 & 982.5 & 30 & 50.7 \\
\hline 1230 & 0.05 & 1000 & 999 & 982.5 & 30 & 50.65 \\
\hline 1235 & 0.00 & 1000 & 1000 & 982.5 & 30 & 50.6 \\
\hline
\end{tabular}

\begin{tabular}{|c|c|c|c|c|}
\hline $\begin{array}{l}\text { Sample } \\
\text { Temperature } \\
{ }^{\circ} \mathrm{C}\end{array}$ & $\begin{array}{l}\text { RPM } \\
\text { Batch } 1 \text { Glass }\end{array}$ & $\%$ Torque & K & $\begin{array}{l}\text { Viscosity } \\
\text { Measured } \\
\text { Poise }\end{array}$ \\
\hline 1198 & 60 & 16.3 & 122.38211 & 33.247139 \\
\hline 1142 & 60 & 23.85 & 125.93553 & 50.059374 \\
\hline 1089 & 60 & 36.45 & 129.29859 & 78.548896 \\
\hline 1035.5 & 60 & 59.05 & 132.69338 & 130.5924 \\
\hline 982.5 & 30 & 50.65 & 136.05645 & 229.70863 \\
\hline
\end{tabular}




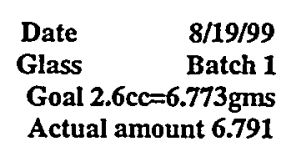

$\begin{array}{ll}\text { TIME } & \text { TIME } \\ \text { MIL } & \text { LEFT }\end{array}$

$\begin{array}{cc}\text { MIL } & \text { LEFT } \\ 1030 & 0.12\end{array}$

$1035 \quad 0.07$

$1040 \quad 0.02$

$1115 \quad 0.12$

$1120 \quad 0.07$

$1125 \quad 0.02$

$1200 \quad 0.12$

$1205 \quad 0.07$

$1210 \quad 0.02$

$1245 \quad 0.12$

$1250 \quad 0.07$

12550.02

1330

1335

1340

0.12

0.07

0.02

SET RATE
${ }^{\circ} \mathrm{C}$
1200
1200
1200
1150
1150
1150
1100
1100
1100
1050
1050
1050
1000
1000
1000

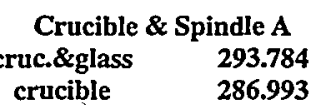

cruc.\&glass $\quad 293.784$

$\begin{array}{lc}\text { glass } & 6.791\end{array}$

FURNACE

SAMPLE

${ }^{\circ} \mathrm{C}$

${ }^{\circ} \mathrm{C}$

$1200 \quad 1199$

1200 . 1199

$1200 \quad 1199$

$1149 \quad 1143$

$1150 \quad 1143.5$

$1150 \quad 1144$

10991090

$1100 \quad 1090$

$1100 \quad 1090$

$1049 \quad 1037$

$1049 \quad 1037$

$1050 \quad 1037$

$999 \quad 984$

$1000 \quad 984$

$1000 \quad 984$

SPEED
RPM

$\%$

TORQUE

16.85

16.85

16.85

25.05

25

25

38.55

38.55

38.55

61.9

61.9

61.9

53.2

52.9

\begin{tabular}{|c|c|c|c|c|}
\hline $\begin{array}{c}\text { Sample } \\
\text { Temperature } \\
{ }^{\circ} \mathrm{C}\end{array}$ & \multicolumn{2}{|c|}{ Batch 1 Glass } & $\mathbf{K}$ & $\begin{array}{c}\text { Viscosity } \\
\text { Measured } \\
\text { Poise }\end{array}$ \\
\hline 1199 & 60 & 16.85 & 122.31865 & 34.351155 \\
\hline 1143.5 & 60 & 25 & 125.84035 & 52.43348 \\
\hline 1090 & 60 & 38.55 & 129.23514 & 83.033577 \\
\hline 1037 & 60 & 61.9 & 132.5982 & 136.79715 \\
\hline 984 & 30 & 52.95 & 135.96126 & 239.97163 \\
\hline
\end{tabular}

52.8 


$\begin{array}{lr}\text { Date } & 8 / 31 / 99 \\ \text { Glass } \quad \text { Batch 1 } \\ \text { Goal 2.6cc=6.773gms } \\ \text { Actual amount } 6.774\end{array}$

TIME TIME

MIL LEFT

$1100 \quad 0.10$
1105

$1105 \quad 0.05$

$1110 \quad 0.00$

$1145 \quad 0.10$

$1150 \quad 0.05$

$1155 \quad 0.00$

1230

1235

1240

1315

1320

1325

1400

1405

1410
0.10

0.05

0.00

0.10

0.05

0.00

0.10

0.05

0.00

Sample

Temperature

${ }^{\circ} \mathrm{C}$

1200

1148

1092

1040

986

\section{RPM \% Torque \\ Batch 1 Glass}

\begin{tabular}{|c|c|}
\hline \multicolumn{2}{|c|}{ Crucible \& Spindle B } \\
\hline cruc.\& & 29 \\
\hline cruc & \\
\hline & \\
\hline
\end{tabular}

SET RATE
${ }_{12}^{\circ} \mathbf{C}$
1200

1200

1200

1150

1150

1150

1100

1100

1100

1050

1050

1050

1000

1000

1000

FURNACE

1200

$1200 \quad 1200$

$1200 \quad 1200$

- 1149

1150

1150

1099

1100

1100

1049

1050

1050

999

1000

1000

16.55
24.3
37.35
59.4
51.4

$\begin{array}{ll}60 & 16.55 \\ 60 & 24.3\end{array}$

60

60

30

51.4

$K$
122.2552
125.55481
129.10823
132.40784
135.83436

Viscosity

Measured

Poise

33.722059

50.849697

80.369874

131.08376

232.72953 


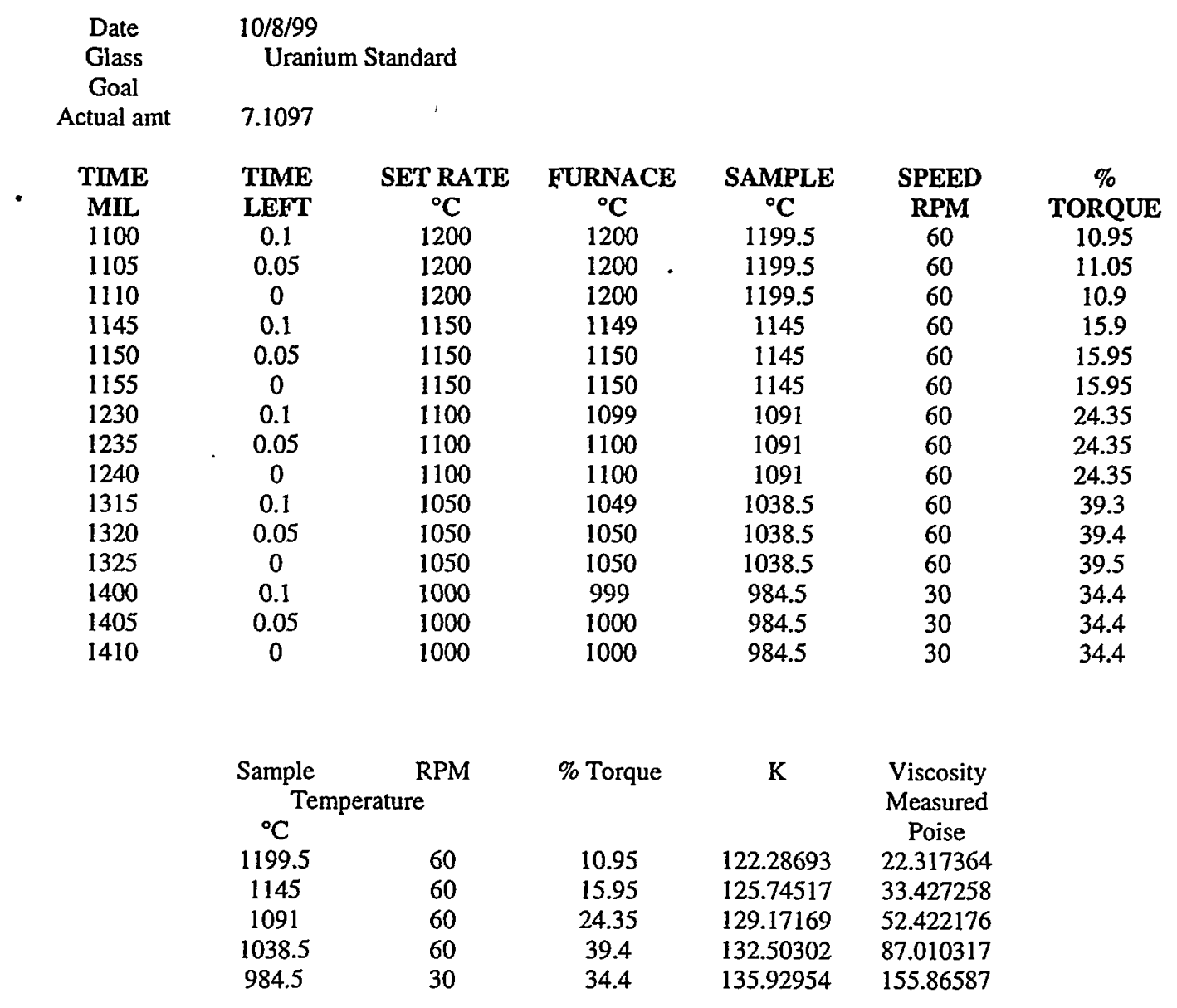




\section{HIM}

\section{1/22/99}

\begin{tabular}{lrr}
\multicolumn{3}{c}{ JMPß Nonlinear Regression } \\
Parameter & Estimate & . \\
ApproxStdErr \\
A & -4.62340847 & 0.63004215 \\
B & 10258.573027 & 1274.44165 \\
C & 61.650376873 & 62.5434012
\end{tabular}

Graph of Fulcher Fit

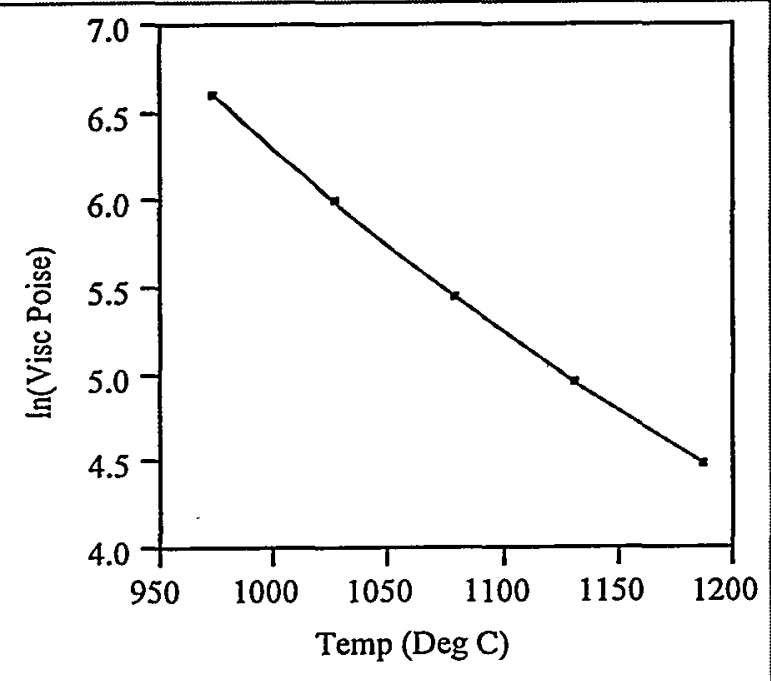

HM

\begin{tabular}{|c|c|c|c|c|}
\hline $\begin{array}{c}\text { Temp } \\
\text { (Deg C) }\end{array}$ & $\begin{array}{c}\text { Measured } \\
\text { Visc. } \\
\text { (Poise) }\end{array}$ & $\begin{array}{c}\text { In Visc } \\
\text { (Fulcher, } \\
\text { Poise) }\end{array}$ & $\begin{array}{c}\text { Measured } \\
\text { InVisc. } \\
\text { (Poise) }\end{array}$ & $\begin{array}{c}\text { Predicted } \\
\text { Visc. } \\
\text { (poise) }\end{array}$ \\
\hline 1187 & 89.64334 & 4.49249 & 4.495839 & 89.34 \\
\hline 1131 & 143.0959 & 4.969874 & 4.963515 & 144.01 \\
\hline 1079 & 235.179 & 5.460217 & 5.460347 & 235.15 \\
\hline 1027 & 406.915 & 6.003387 & 6.008604 & 404.80 \\
\hline 974 & 748.7312 & 6.620716 & 6.61838 & 750.48 \\
\hline $\mathbf{1 1 5 0}$ & $\mathbf{?}$ & $\mathbf{4 . 8 0 2 3 9 8}$ & $\mathbf{?}$ & $\mathbf{1 2 1 . 8}$ \\
\hline & $\mathbf{?}$ & & & \\
\hline
\end{tabular}




\section{HM}

\section{$1 / 28 / 99$}

\begin{tabular}{lrr}
\multicolumn{3}{c}{ JMP® ${ }^{\text {Nonlinear Regression }}$} \\
Parameter & $\begin{array}{r}\text { Estimate } \\
\text { ApproxStdErr }\end{array}$ \\
A & -4.582908606 & 0.35512756 \\
B & 10167.71155 & 715.479671 \\
C & 67.374106932 & 35.2822506
\end{tabular}

\section{Graph of Fulcher Fit}

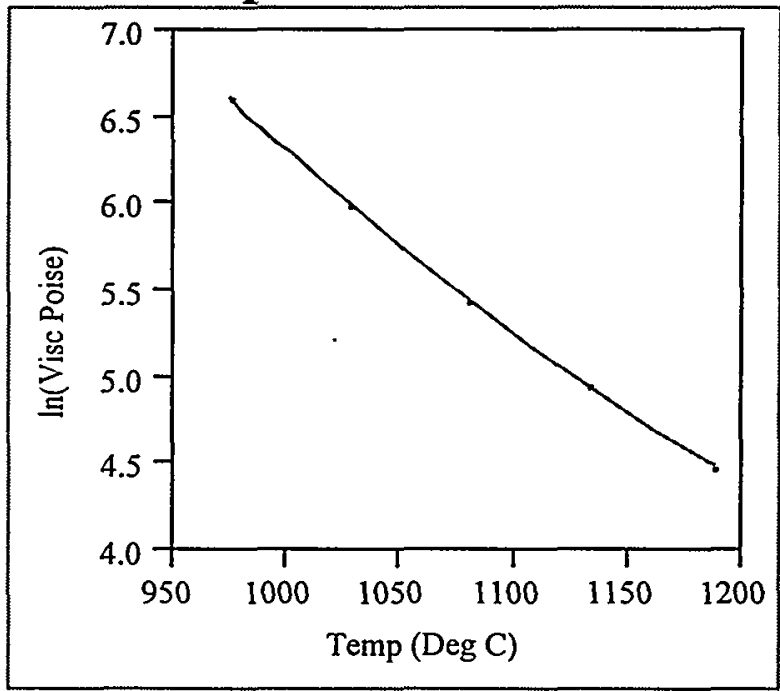

HM 1

\begin{tabular}{|c|c|c|c|c|}
\hline $\begin{array}{c}\text { Temp } \\
\text { (Deg C) }\end{array}$ & $\begin{array}{c}\text { Measured } \\
\text { Visc. } \\
\text { (Poise) }\end{array}$ & $\begin{array}{c}\text { In Visc } \\
\text { (Fulcher, } \\
\text { Poise) }\end{array}$ & $\begin{array}{c}\text { Measured } \\
\text { InVisc. } \\
\text { (Poise) }\end{array}$ & $\begin{array}{c}\text { Predicted } \\
\text { Visc. } \\
\text { (poise) }\end{array}$ \\
\hline 1188 & 88.98204 & 4.490335 & 4.488435 & 89.15 \\
\hline 1134 & 141.6163 & 4.949685 & 4.953122 & 141.13 \\
\hline 1081 & 232.3531 & 5.448121 & 5.448258 & 232.32 \\
\hline 1028 & 402.8371 & 6.001557 & 5.998532 & 404.06 \\
\hline 976 & 741.481 & 6.607298 & 6.60865 & 740.48 \\
\hline $\mathbf{1 1 5 0}$ & $\mathbf{?}$ & $\mathbf{4 . 8 0 8 8 0 4}$ & $\mathbf{?}$ & $\mathbf{1 2 2 . 5 8}$ \\
\hline & - & & & \\
\hline
\end{tabular}


Purex

2/2/99

\begin{tabular}{lrr}
\multicolumn{3}{c}{ JMP® Nonlinear Regression } \\
Parameter & Estimate & ApproxStdErr \\
A & -3.605459573 & 0.30455242 \\
B & 6349.3945382 & 523.880195 \\
C & 212.03562655 & 35.2584469
\end{tabular}

Graph of Fulcher Fit

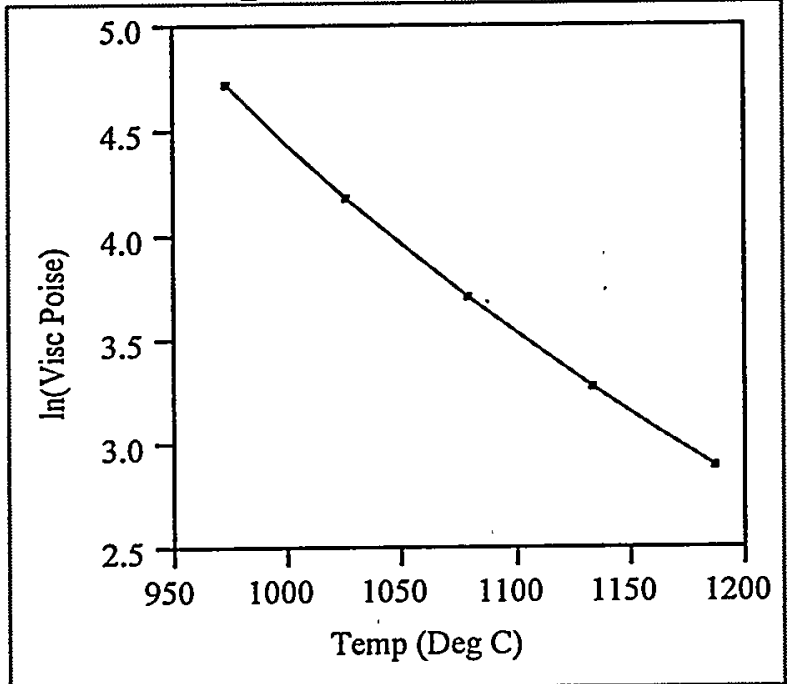

Purex

\begin{tabular}{|c|c|c|c|c|}
\hline $\begin{array}{c}\text { Temp } \\
\text { (Deg C) }\end{array}$ & $\begin{array}{c}\text { Measured } \\
\text { Visc. } \\
\text { (Poise) }\end{array}$ & $\begin{array}{c}\text { In Visc } \\
\text { (Fulcher, } \\
\text { Poise) }\end{array}$ & $\begin{array}{c}\text { Measured } \\
\text { InVisc. } \\
\text { (Poise) }\end{array}$ & $\begin{array}{c}\text { Predicted } \\
\text { Visc. } \\
\text { (poise) }\end{array}$ \\
\hline 1187 & 18.2569 & 2.906978 & 2.904543 & 18.30 \\
\hline 1134 & 26.738 & 3.281352 & 3.286086 & 26.61 \\
\hline 1079 & 41.1455 & 3.718249 & 3.717115 & 41.19 \\
\hline 1026 & 66.2038 & 4.195121 & 4.192738 & 66.36 \\
\hline 974 & 113.1469 & 4.727469 & 4.728687 & 113.01 \\
\hline $\mathbf{1 1 5 0}$ & $\mathbf{?}$ & $\mathbf{3 . 1 6 3 8 7 5}$ & $\mathbf{?}$ & $\mathbf{2 3 . 6 6}$ \\
\hline & - & & & \\
\hline
\end{tabular}


Purex

2/5/99

\section{JMP® Nonlinear Regression \\ Parameter Estimate \\ A \\ $-3.898484758$ \\ 6788.1400585 \\ 181.45202066 \\ ApproxStdErr \\ 0.13987201 \\ 249.207654 \\ 16.2567964}

\section{Graph of Fulcher Fit}

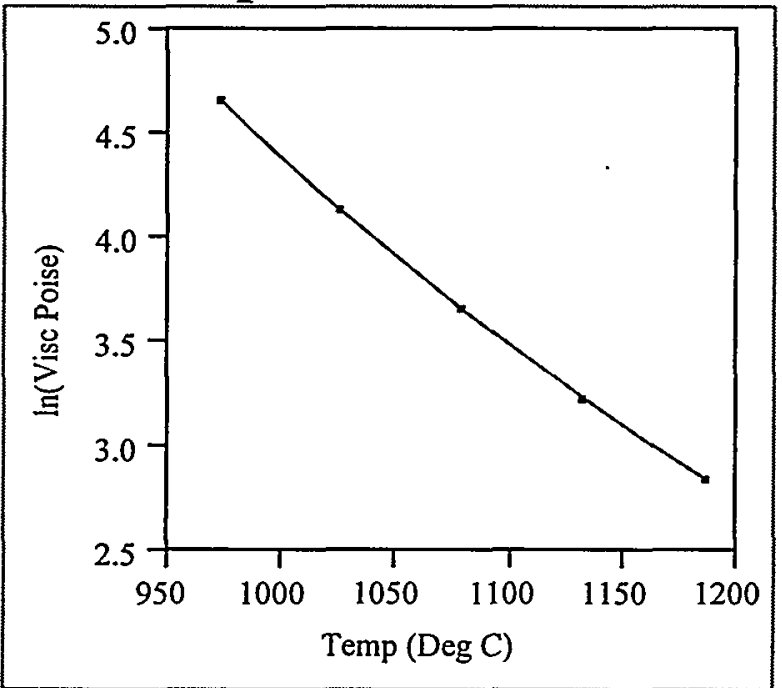

Purex

\begin{tabular}{|c|c|c|c|c|}
\hline $\begin{array}{c}\text { Temp } \\
\text { (Deg C) }\end{array}$ & $\begin{array}{c}\text { Measured } \\
\text { Visc. } \\
\text { (Poise) }\end{array}$ & $\begin{array}{c}\text { In Visc } \\
\text { (Fulcher, } \\
\text { Poise) }\end{array}$ & $\begin{array}{c}\text { Measured } \\
\text { InVisc. } \\
\text { (Poise) }\end{array}$ & $\begin{array}{c}\text { Predicted } \\
\text { Visc. } \\
\text { (poise) }\end{array}$ \\
\hline 1187 & 17.3338 & 2.852203 & 2.852658 & 17.33 \\
\hline 1133 & 25.4067 & 3.235302 & 3.235013 & 25.41 \\
\hline 1079 & 38.9799 & 3.664498 & 3.663046 & 39.04 \\
\hline 1026 & 62.8714 & 4.139117 & 4.141091 & 62.75 \\
\hline 974 & 106.2488 & 4.666473 & 4.665784 & 106.32 \\
\hline $\mathbf{1 1 5 0}$ & $\mathbf{?}$ & $\mathbf{3 . 1 1 0 0 8 9}$ & $\mathbf{?}$ & $\mathbf{2 2 . 4 2}$ \\
\hline & & & & \\
\hline
\end{tabular}




\section{Batch 1}

2/9/99

\begin{tabular}{lrr}
\multicolumn{2}{c}{ JMP® Nonlinear Regression } \\
Parameter & $\begin{array}{r}\text { Estimate } \\
\text { ApproxStdErr }\end{array}$ \\
A & -4.494750139 & 0.61567372 \\
B & 8724.7982887 & 1188.84714 \\
C & 108.53429464 & 65.4570676
\end{tabular}

Graph of Fulcher Fit

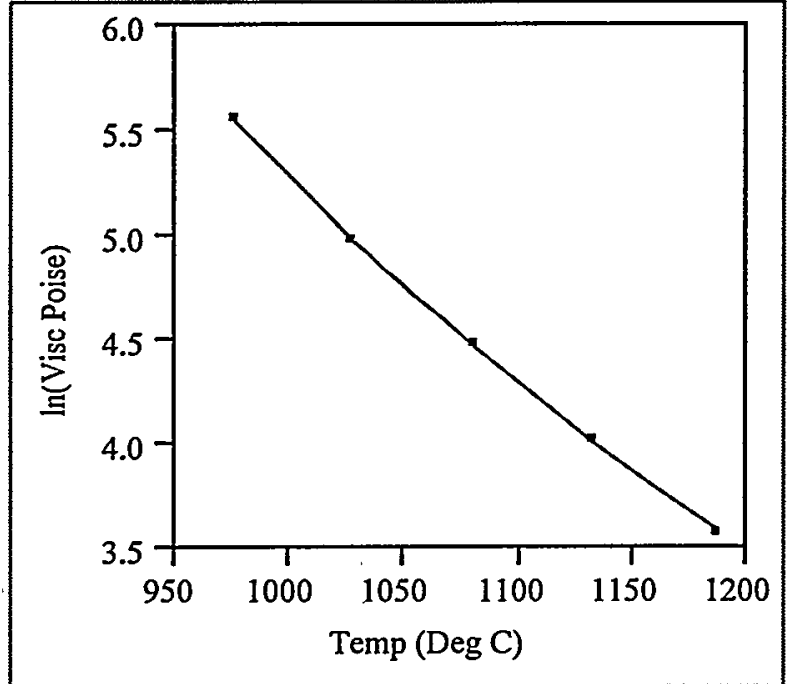

Batch 1

\begin{tabular}{|c|c|c|c|c|}
\hline $\begin{array}{c}\text { Temp } \\
\text { (Deg C) }\end{array}$ & $\begin{array}{c}\text { Measured } \\
\text { Visc. } \\
\text { (Poise) }\end{array}$ & $\begin{array}{c}\text { In Visc } \\
\text { (Fulcher, } \\
\text { Poise) }\end{array}$ & $\begin{array}{c}\text { Measured } \\
\text { InVisc. } \\
\text { (Poise) }\end{array}$ & $\begin{array}{c}\text { Predicted } \\
\text { Visc. } \\
\text { (poise) }\end{array}$ \\
\hline 1187 & 36.30863 & 3.59526 & 3.592055 & 36.43 \\
\hline 1133 & 56.0846 & 4.021687 & 4.026861 & 55.80 \\
\hline 1080 & 88.96073 & 4.486317 & 4.488195 & 88.79 \\
\hline 1027 & 148.1104 & 5.004568 & 4.997958 & 149.09 \\
\hline 976 & 261.3379 & 5.563052 & 5.565814 & 260.62 \\
\hline $\mathbf{1 1 5 0}$ & $\mathbf{?}$ & $\mathbf{3 . 8 8 2 6 7 2}$ & $\mathbf{?}$ & $\mathbf{4 8 . 5 5}$ \\
\hline & $\mathbf{r}$ & & & \\
\hline
\end{tabular}




\section{Batch 1}

2/17/99

\begin{tabular}{lrr}
\multicolumn{3}{c}{ JMP® Nonlinear Regression } \\
Parameter & Estimate & ApproxStdErr \\
A & -4.368131388 & 0.24007985 \\
B & 8555.4053557 & 460.183922 \\
C & 115.94617024 & 25.6476823
\end{tabular}

Graph of Fulcher Fit

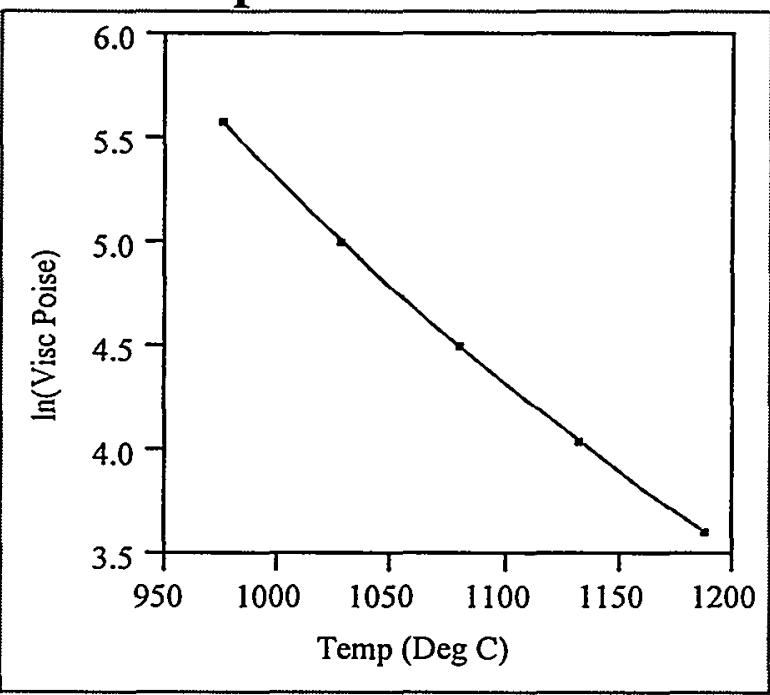

Batch 1

\begin{tabular}{|c|c|c|c|c|}
\hline $\begin{array}{c}\text { Temp } \\
\text { (Deg C) }\end{array}$ & $\begin{array}{c}\text { Measured } \\
\text { Visc. } \\
\text { (Poise) }\end{array}$ & $\begin{array}{c}\text { In Visc } \\
\text { (Fulcher, } \\
\text { Poise) }\end{array}$ & $\begin{array}{c}\text { Measured } \\
\text { lnVisc. } \\
\text { (Poise) }\end{array}$ & $\begin{array}{c}\text { Predicted } \\
\text { Visc. } \\
\text { (poise) }\end{array}$ \\
\hline 1188 & 37.00751 & 3.612256 & 3.611121 & 37.05 \\
\hline 1133 & 57.13882 & 4.043818 & 4.045484 & 57.04 \\
\hline 1080 & 90.69233 & 4.506275 & 4.507473 & 90.58 \\
\hline 1028 & 149.8155 & 5.012242 & 5.009404 & 150.24 \\
\hline 976 & 265.2045 & 5.579392 & 5.580501 & 264.91 \\
\hline $\mathbf{1 1 5 0}$ & $\mathbf{?}$ & $\mathbf{3 . 9 0 5 5 2 4}$ & $\boldsymbol{?}$ & $\mathbf{4 9 . 6 8}$ \\
\hline & - & & & \\
\hline
\end{tabular}




\section{Glass 2}

\section{2/24/99}

\begin{tabular}{lrr}
\multicolumn{3}{c}{ JMP® ${ }^{\text {Nonlinear Regression }}$} \\
Parameter & Estimate & ApproxStdErr \\
A & -4.189857342 & 1.03450359 \\
B & 8245.3057008 & 1876.67085 \\
C & 165.8200733 & 102.631977
\end{tabular}

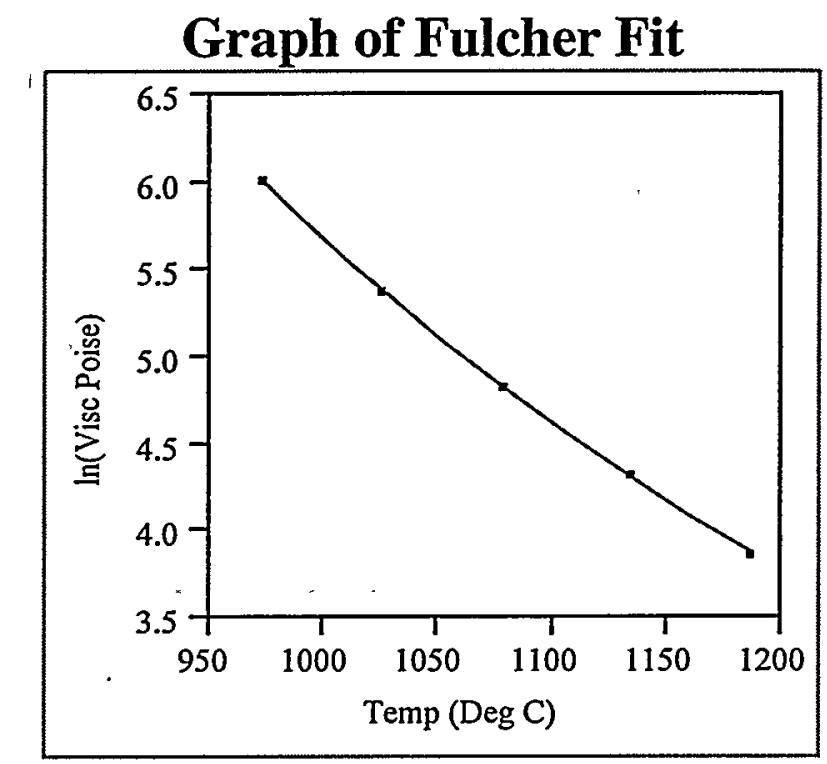

Glass 2

\begin{tabular}{|c|c|c|c|c|}
\hline $\begin{array}{c}\text { Temp } \\
\text { (Deg C) }\end{array}$ & $\begin{array}{c}\text { Measured } \\
\text { Visc. } \\
\text { (Poise) }\end{array}$ & $\begin{array}{c}\text { In Visc } \\
\text { (Fulcher, } \\
\text { Poise) }\end{array}$ & $\begin{array}{c}\text { Measured } \\
\text { InVisc. } \\
\text { (Poise) }\end{array}$ & $\begin{array}{c}\text { Predicted } \\
\text { Visc. } \\
\text { (poise) }\end{array}$ \\
\hline 1187.5 & 48.09141 & 3.880484 & 3.873104 & 48.45 \\
\hline 1135 & 76.14377 & 4.31765 & 4.332623 & 75.01 \\
\hline 1079 & 125.7103 & 4.839366 & 4.83398 & 126.39 \\
\hline 1026 & 219.2722 & 5.395702 & 5.390314 & 220.46 \\
\hline 974 & 409.7874 & 6.012457 & 6.015639 & 408.49 \\
\hline $\mathbf{1 1 5 0}$ & $\mathbf{?}$ & $\mathbf{4 . 1 8 7 9 8 6}$ & $\mathbf{?}$ & $\mathbf{6 5 . 8 9}$ \\
\hline & $\mathbf{}$ & & & \\
\hline
\end{tabular}




\section{Glass 4}

$3 / 2 / 99$

$\begin{array}{lrr}\text { JMP® Nonlinear Regression } \\ \text { Parameter } & \begin{array}{r}\text { Estimate } \\ \text { ApproxStdErr }\end{array} \\ \text { A } & -5.021262968 & 1.00618082 \\ \text { B } & 10407.799087 & 2018.70962 \\ \text { C } & 71.368094278 & 96.8389453\end{array}$

Graph of Fulcher Fit

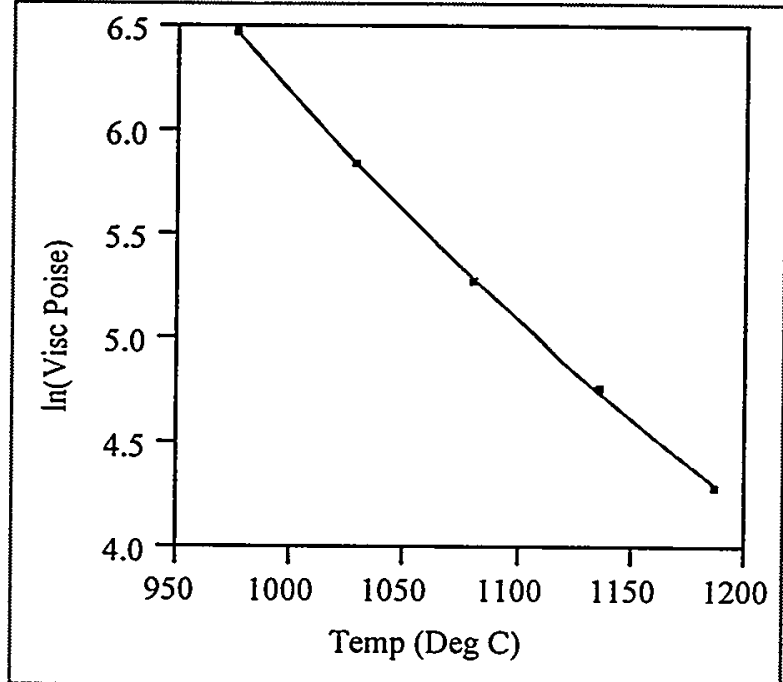

Glass 4

\begin{tabular}{|c|c|c|c|c|}
\hline $\begin{array}{c}\text { Temp } \\
\text { (Deg C) }\end{array}$ & $\begin{array}{c}\text { Measured } \\
\text { Visc. } \\
\text { (Poise) }\end{array}$ & $\begin{array}{c}\text { In Visc } \\
\text { (Fulcher, } \\
\text { Poise) }\end{array}$ & $\begin{array}{c}\text { Measured } \\
\text { InVisc. } \\
\text { (Poise) }\end{array}$ & $\begin{array}{c}\text { Predicted } \\
\text { Visc. } \\
\text { (poise) }\end{array}$ \\
\hline 1187 & 73.84806 & 4.307799 & 4.30201 & 74.28 \\
\hline 1136 & 117.4741 & 4.754697 & 4.766218 & 116.13 \\
\hline 1081 & 197.0891 & 5.287246 & 5.283656 & 197.80 \\
\hline 1028 & 348.4596 & 5.858365 & 5.853522 & 350.15 \\
\hline 976 & 656.1879 & 6.483747 & 6.486447 & 654.42 \\
\hline $\mathbf{1 1 5 0}$ & $\mathbf{?}$ & $\mathbf{4 . 6 2 7 8 1 1}$ & $\mathbf{?}$ & $\mathbf{1 0 2 . 2 9}$ \\
\hline & & & & \\
\hline
\end{tabular}




\section{Glass 2}

$3 / 4 / 99$

\begin{tabular}{lrr}
\multicolumn{3}{c}{. JMP® Nonlinear Regression } \\
Parameter & Estimate & ApproxStdErr \\
A & -3.425115457 & 0.24581215 \\
B & 6856.6345785 & 404.535923 \\
C & 248.27043953 & 24.1101762
\end{tabular}

Graph of Fulcher Fit

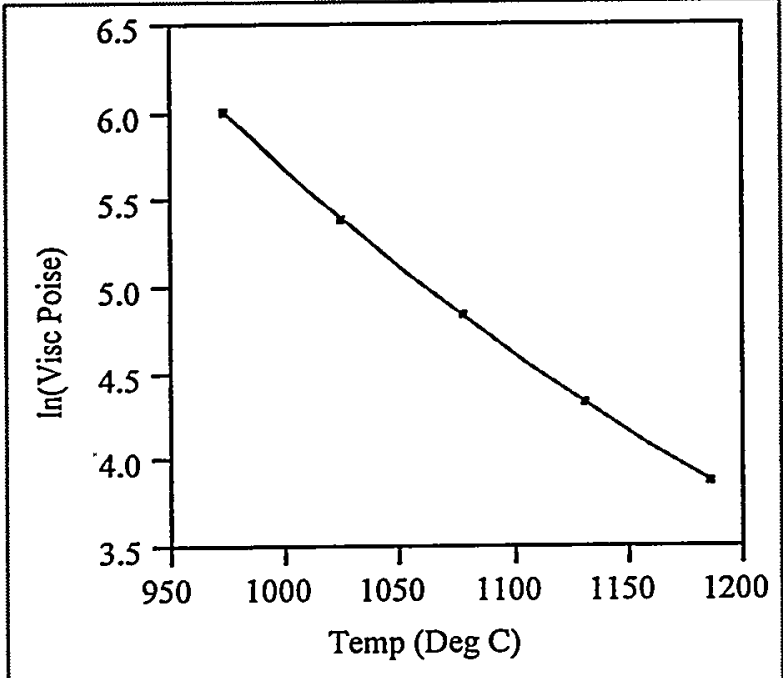

Glass 2

\begin{tabular}{|c|c|c|c|c|}
\hline $\begin{array}{c}\text { Temp } \\
\text { (Deg C) }\end{array}$ & $\begin{array}{c}\text { Measured } \\
\text { Visc. } \\
\text { (Poise) }\end{array}$ & $\begin{array}{c}\text { In Visc } \\
\text { (Fulcher, } \\
\text { Poise) }\end{array}$ & $\begin{array}{c}\text { Measured } \\
\text { InVisc. } \\
\text { (Poise) }\end{array}$ & $\begin{array}{c}\text { Predicted } \\
\text { Visc. } \\
\text { (poise) }\end{array}$ \\
\hline 1186 & 48.74432 & 3.886838 & 3.886589 & 48.76 \\
\hline 1131 & 76.82434 & 4.342422 & 4.341522 & 76.89 \\
\hline 1078 & 126.7467 & 4.838583 & 4.84219 & 126.29 \\
\hline 1025 & 221.1547 & 5.402455 & 5.398863 & 221.95 \\
\hline 974 & 413.2023 & 6.022804 & 6.023937 & 412.73 \\
\hline $\mathbf{1 1 5 0}$ & $\mathbf{?}$ & $\mathbf{4 . 1 7 8 7 5 5}$ & $\mathbf{?}$ & $\mathbf{6 5 . 2 8}$ \\
\hline & - & & & \\
\hline
\end{tabular}




\section{Glass 4}

3/9/99

\begin{tabular}{lrr}
\multicolumn{2}{c}{ JMP® } & $\begin{array}{r}\text { Nonlinear Regression } \\
\text { Estimate }\end{array}$ \\
Parameter & -4.084227367 & ApproxStdErr \\
A & 8668.993743 & 0.68012829 \\
B & 155.1141533 & 1244.73887 \\
C & & 65.2453892
\end{tabular}

Graph of Fulcher Fit

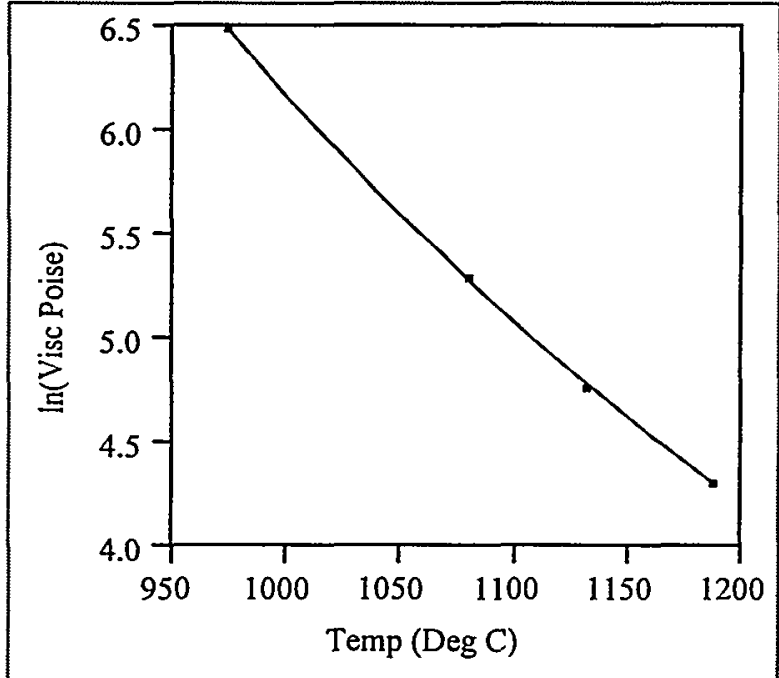

Glass 4

\begin{tabular}{|c|c|c|c|c|}
\hline $\begin{array}{c}\text { Temp } \\
\text { (Deg C) }\end{array}$ & $\begin{array}{c}\text { Measured } \\
\text { Visc. } \\
\text { (Poise) }\end{array}$ & $\begin{array}{c}\text { In Visc } \\
\text { (Fulcher, } \\
\text { Poise) }\end{array}$ & $\begin{array}{c}\text { Measured } \\
\text { InVisc. } \\
\text { (Poise) }\end{array}$ & $\begin{array}{c}\text { Predicted } \\
\text { Visc. } \\
\text { (poise) }\end{array}$ \\
\hline 1188 & 74.52759 & 4.308756 & 4.311169 & 74.35 \\
\hline 1133 & 118.4945 & 4.780809 & 4.774867 & 119.20 \\
\hline 1080 & 198.9171 & 5.288814 & 5.292888 & 198.11 \\
\hline 975 & 657.6308 & 6.489188 & 6.488644 & 657.99 \\
\hline $\mathbf{1 1 5 0}$ & $\boldsymbol{?}$ & $\mathbf{4 . 6 2 9 3 2 9}$ & $\boldsymbol{?}$ & $\mathbf{1 0 2 . 4 5}$ \\
\hline & & & & \\
\hline & - & & & \\
\hline
\end{tabular}




\section{Glass 3} 3/31/99

\begin{tabular}{lrr}
\multicolumn{2}{c}{ JMP® Nonlinear Regression } \\
Parameter & Estimate & ApproxStdErr \\
A & -5.203487644 & 2.01681083 \\
B & 11025.282625 & 4234.6444 \\
C & 24.135611033 & 200.696009
\end{tabular}

Graph of Fulcher Fit

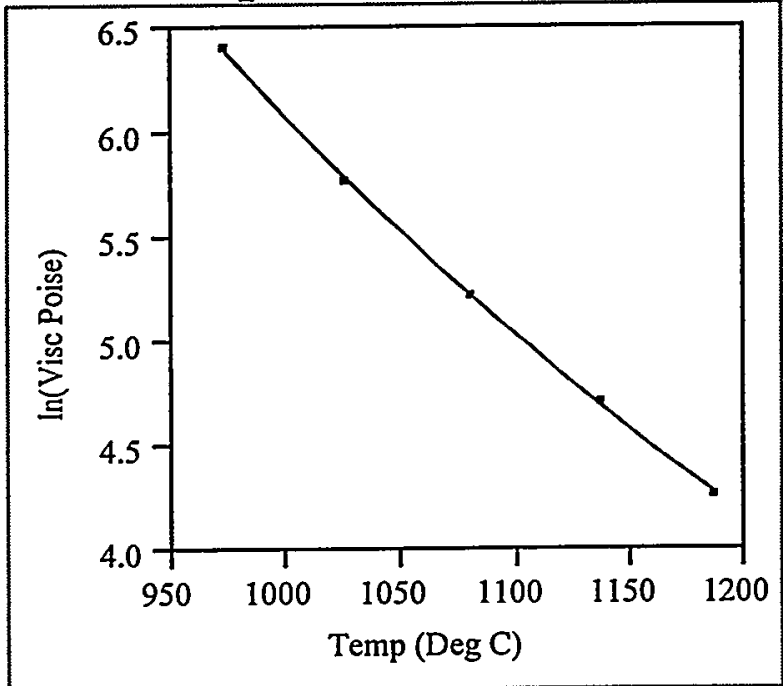

Glass 3

\begin{tabular}{|c|c|c|c|c|}
\hline $\begin{array}{c}\text { Temp } \\
\text { (Deg C) }\end{array}$ & $\begin{array}{c}\text { Measured } \\
\text { Visc. } \\
\text { (Poise) }\end{array}$ & $\begin{array}{c}\text { In Visc } \\
\text { (Fulcher, } \\
\text { Poise) }\end{array}$ & $\begin{array}{c}\text { Measured } \\
\text { InVisc. } \\
\text { (Poise) }\end{array}$ & $\begin{array}{c}\text { Predicted } \\
\text { Visc. } \\
\text { (poise) }\end{array}$ \\
\hline 1187 & 71.28389 & 4.277655 & 4.26667 & 72.07 \\
\hline 1137 & 112.5754 & 4.703634 & 4.723623 & 110.35 \\
\hline 1080.5 & 186.9667 & 5.233519 & 5.23093 & 187.45 \\
\hline 1026 & 326.5757 & 5.801278 & 5.788662 & 330.72 \\
\hline 974 & 607.8513 & 6.40373 & 6.40993 & 604.09 \\
\hline $\mathbf{1 1 5 0}$ & $\mathbf{?}$ & $\mathbf{4 . 5 8 9 2 3 9}$ & $\mathbf{?}$ & $\mathbf{9 8 . 4 2}$ \\
\hline & - & & & \\
\hline
\end{tabular}




$\begin{array}{lrr}\text { JMP } \AA \text { Nonlinear Regression } \\ \text { Parameter } & \begin{array}{r}\text { Estimate } \\ \text { ApproxStdErr }\end{array} \\ \text { A } & -5.043143248 & 0.17175151 \\ \text { B } & 11083.92262 & 361.695094 \\ \text { C } & 24.344662735 & 17.1110281\end{array}$

\section{Graph of Fulcher Fit}

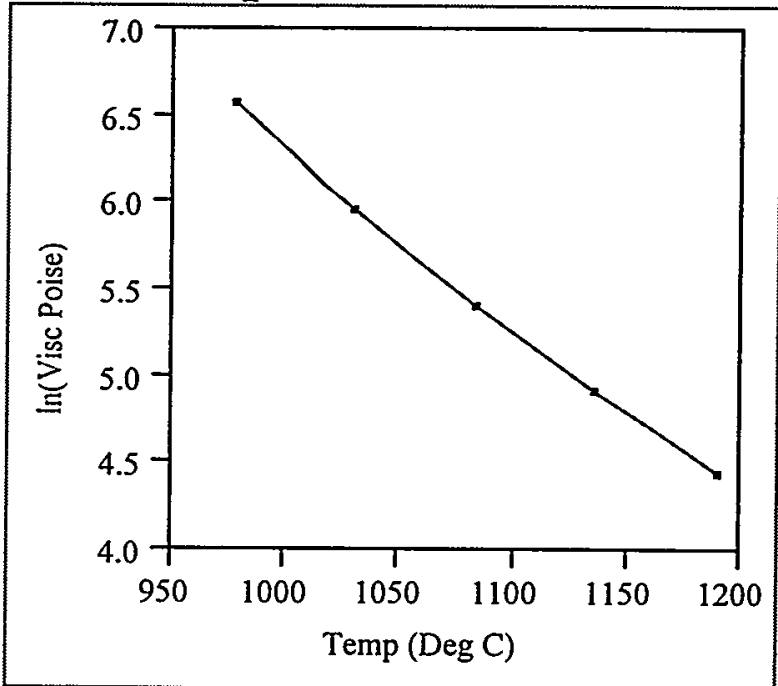

HM

\begin{tabular}{|c|c|c|c|c|}
\hline $\begin{array}{c}\text { Temp } \\
\text { (Deg C) }\end{array}$ & $\begin{array}{c}\text { Measured } \\
\text { Visc. } \\
\text { (Poise) }\end{array}$ & $\begin{array}{c}\text { In Visc } \\
\text { (Fulcher, } \\
\text { Poise) }\end{array}$ & $\begin{array}{c}\text { Measured } \\
\text { InVisc. } \\
\text { (Poise) }\end{array}$ & $\begin{array}{c}\text { Predicted } \\
\text { Visc. } \\
\text { (poise) }\end{array}$ \\
\hline 1191 & 86.28547 & 4.457454 & 4.457661 & 86.27 \\
\hline 1136 & 137.8952 & 4.927503 & 4.926494 & 138.03 \\
\hline 1084 & 225.5316 & 5.416789 & 5.41846 & 225.15 \\
\hline 1031 & 390.0715 & 5.9675 & 5.96633 & 390.53 \\
\hline 978 & 720.3402 & 6.579423 & 6.579724 & 720.12 \\
\hline $\mathbf{1 1 5 0}$ & $\boldsymbol{?}$ & $\mathbf{4 . 8 0 3 4 9 7}$ & $\boldsymbol{?}$ & $\mathbf{1 2 1 . 9 4}$ \\
\hline & & & & \\
\hline
\end{tabular}




\section{Glass 1}

4/12/99

\begin{tabular}{lrr}
\multicolumn{3}{c}{ JMP® ${ }^{\text {Nonlinear Regression }}$} \\
Parameter & $\begin{array}{r}\text { Estimate } \\
\text { ApproxStdErr }\end{array}$ \\
A & -5.251703083 & 0.25159913 \\
B & 10720.570572 & 516.056657 \\
C & 48.707616219 & 24.5749404
\end{tabular}

Graph of Fulcher Fit

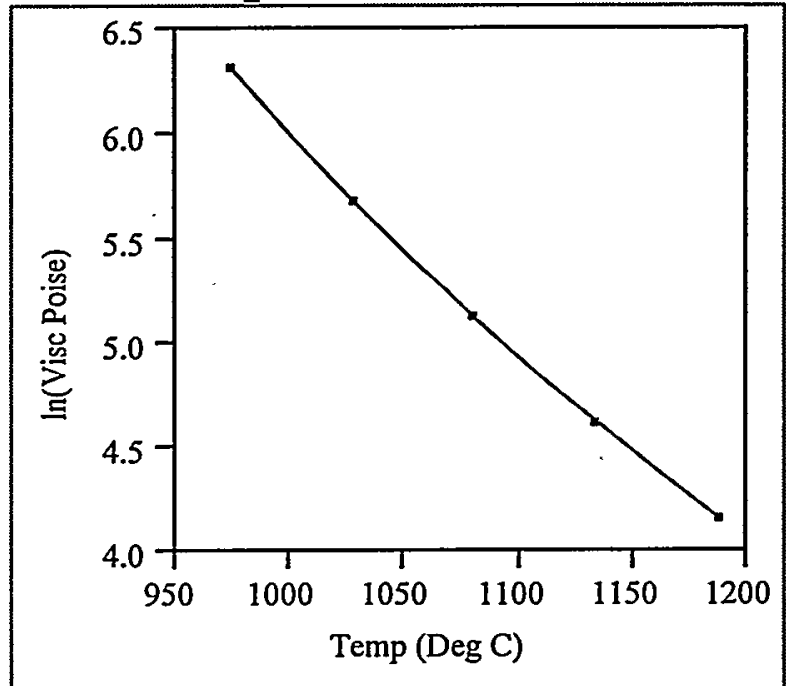

Glass 1

\begin{tabular}{|c|c|c|c|c|}
\hline $\begin{array}{c}\text { Temp } \\
\text { (Deg C) }\end{array}$ & $\begin{array}{c}\text { Measured } \\
\text { Visc. } \\
\text { (Poise) }\end{array}$ & $\begin{array}{c}\text { In Visc } \\
\text { (Fulcher, } \\
\text { Poise) }\end{array}$ & $\begin{array}{c}\text { Measured } \\
\text { InVisc. } \\
\text { (Poise) }\end{array}$ & $\begin{array}{c}\text { Predicted } \\
\text { Visc. } \\
\text { (poise) }\end{array}$ \\
\hline 1188 & 63.96866 & 4.158147 & 4.158393 & 63.95 \\
\hline 1134 & 101.9975 & 4.626345 & 4.624948 & 102.14 \\
\hline 1081 & 170.0462 & 5.133504 & 5.13607 & 169.61 \\
\hline 1028 & 296.9675 & 5.695559 & 5.693623 & 297.54 \\
\hline 975 & 556.9382 & 6.321933 & 6.322454 & 556.65 \\
\hline 1150 & $\mathbf{?}$ & 4.482833 & $\mathbf{?}$ & $\mathbf{8 8 . 4 9}$ \\
\hline & $\mathbf{2}$ & & & \\
\hline
\end{tabular}




\section{Glass 5 4/15/99}

\begin{tabular}{lrr}
\multicolumn{2}{c}{ IMPß Nonlinear Regression } \\
Parameter & $\begin{array}{r}\text { Estimate } \\
\text { ApproxStdErr }\end{array}$ \\
A & -4.513995046 & 0.95575278 \\
B & 9651.1515193 & 1862.32804 \\
C & 99.882043264 & 93.5353944
\end{tabular}

Graph of Fulcher Fit

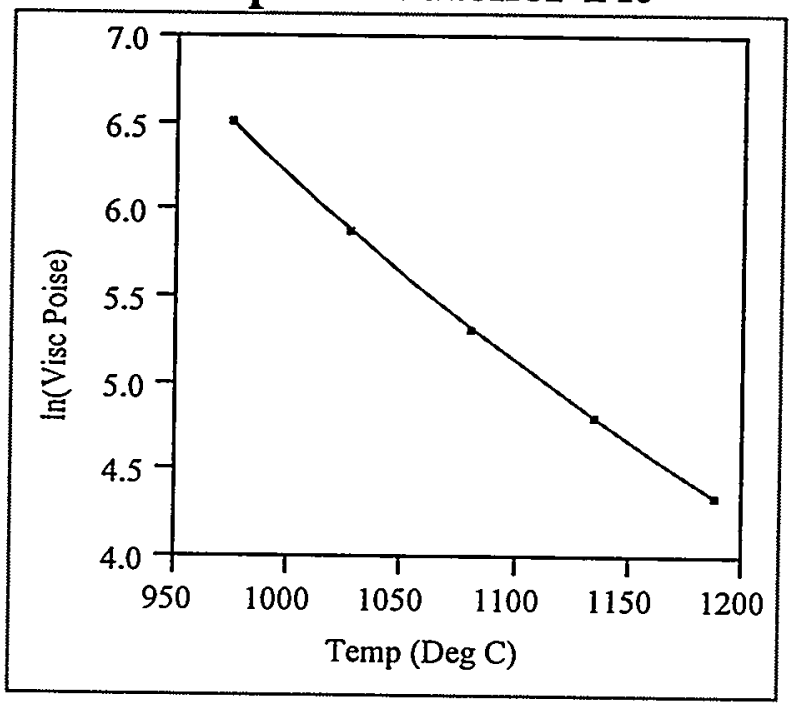

Glass 1

\begin{tabular}{|c|c|c|c|c|}
\hline $\begin{array}{c}\text { Temp } \\
\text { (Deg C) }\end{array}$ & $\begin{array}{c}\text { Measured } \\
\text { Visc. } \\
\text { (Poise) }\end{array}$ & $\begin{array}{c}\text { In Visc } \\
\text { (Fulcher, } \\
\text { Poise) }\end{array}$ & $\begin{array}{c}\text { Measured } \\
\text { lnVisc. } \\
\text { (Poise) }\end{array}$ & $\begin{array}{c}\text { Predicted } \\
\text { Visc. } \\
\text { (poise) }\end{array}$ \\
\hline 1188 & 77.50049 & 4.355587 & 4.350284 & 77.91 \\
\hline 1135.5 & 123.6104 & 4.805225 & 4.817135 & 122.15 \\
\hline 1080 & 205.627 & 5.332934 & 5.326064 & 207.04 \\
\hline 1027 & 363.0592 & 5.895847 & 5.894566 & 363.52 \\
\hline 975 & 675.8351 & 6.514406 & 6.515949 & 674.79 \\
\hline $\mathbf{1 1 5 0}$ & $\mathbf{?}$ & $\mathbf{4 . 6 7 6 5 4 5}$ & $\mathbf{?}$ & $\mathbf{1 0 7 . 4 0}$ \\
\hline &.. & & & \\
\hline
\end{tabular}


Glass 3

$4 / 19 / 99$

\begin{tabular}{lrr}
\multicolumn{3}{c}{ JMP® ${ }^{\text {Nonlinear Regression }}$} \\
Parameter & $\begin{array}{c}\text { Estimate } \\
\text { ApproxStdErr }\end{array}$ \\
A & -4.275829886 & 1.16787639 \\
B & 9234.5665897 & 2234.14731 \\
C & 118.44944286 & 115.103158
\end{tabular}

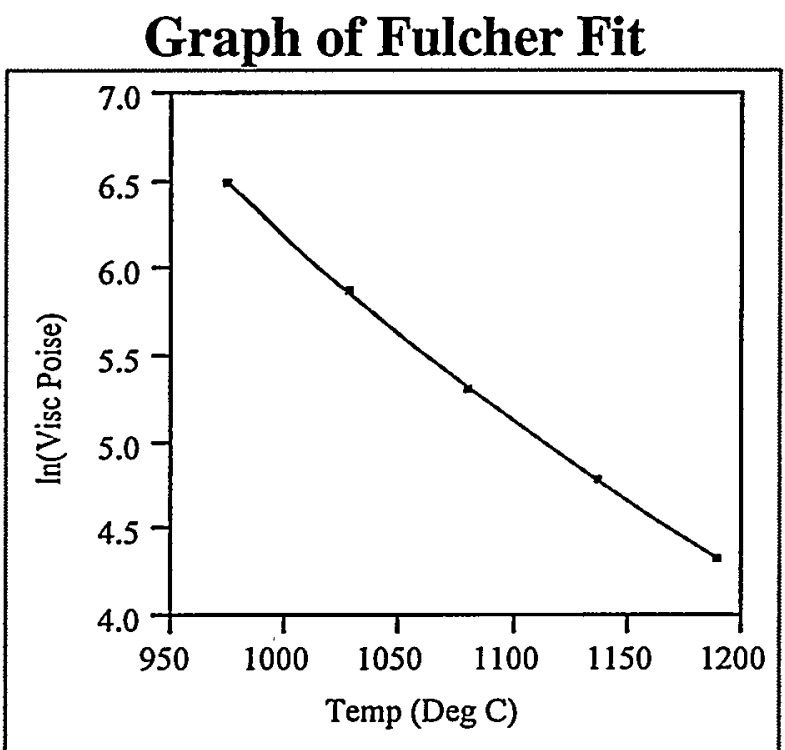

Glass 3

\begin{tabular}{|c|c|c|c|c|}
\hline $\begin{array}{c}\text { Temp } \\
\text { (Deg C) }\end{array}$ & $\begin{array}{c}\text { Measured } \\
\text { Visc. } \\
\text { (Poise) }\end{array}$ & $\begin{array}{c}\text { In Visc } \\
\text { (Fulcher, } \\
\text { Poise) }\end{array}$ & $\begin{array}{c}\text { Measured } \\
\text { InVisc. } \\
\text { (Poise) }\end{array}$ & $\begin{array}{c}\text { Predicted } \\
\text { Visc. } \\
\text { (poise) }\end{array}$ \\
\hline 1190 & 76.49886 & 4.342118 & 4.337276 & 76.87 \\
\hline 1137 & 121.9392 & 4.79055 & 4.803522 & 120.37 \\
\hline 1080 & 203.4625 & 5.327999 & 5.315482 & 206.03 \\
\hline 1028 & 358.4473 & 5.87706 & 5.881782 & 356.76 \\
\hline 975 & 668.4396 & 6.505281 & 6.504946 & 668.66 \\
\hline $\mathbf{1 1 5 0}$ & $\mathbf{?}$ & $\mathbf{4 . 6 7 6 2 9 2}$ & $\mathbf{?}$ & $\mathbf{1 0 7 . 3 7}$ \\
\hline & & & & \\
\hline
\end{tabular}




\section{Glass 5} $4 / 27 / 99$

$\begin{array}{lrr}\text { Parameter } & \begin{array}{r}\text { Nonlinear Regression } \\ \text { Estimate }\end{array} & \begin{array}{r}\text { ApproxStdErr } \\ \text { ApP }\end{array} \\ \text { A } & -4.47062997 & 0.38089472 \\ \text { B } & 9636.0868103 & 744.068783 \\ \text { C } & 98.661509029 & 37.5229028\end{array}$

\section{Graph of Fulcher Fit}

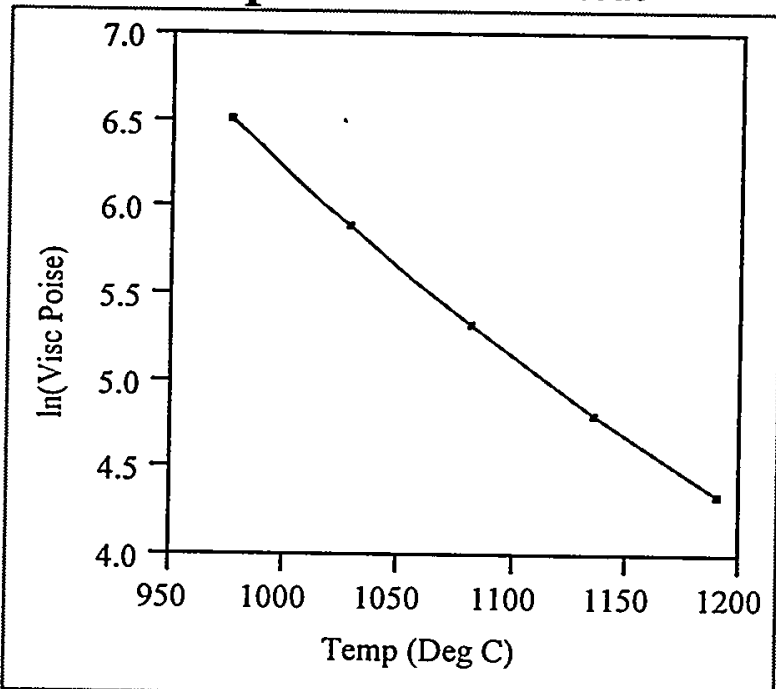

Glass 5

\begin{tabular}{|c|c|c|c|c|}
\hline $\begin{array}{c}\text { Temp } \\
\text { (Deg C) }\end{array}$ & $\begin{array}{c}\text { Measured } \\
\text { Visc. } \\
\text { (Poise) }\end{array}$ & $\begin{array}{c}\text { ln Visc } \\
\text { (Fulcher, } \\
\text { Poise) }\end{array}$ & $\begin{array}{c}\text { Measured } \\
\text { InVisc. } \\
\text { (Poise) }\end{array}$ & $\begin{array}{c}\text { Predicted } \\
\text { Visc. } \\
\text { (poise) }\end{array}$ \\
\hline 1190.5 & 77.70769 & 4.354931 & 4.352954 & 77.86 \\
\hline 1136.3 & 124.0449 & 4.815925 & 4.820644 & 123.46 \\
\hline 1081.5 & 206.5577 & 5.333714 & 5.33058 & 207.21 \\
\hline 1028 & 364.329 & 5.898129 & 5.898057 & 364.36 \\
\hline 975.5 & 678.1806 & 6.51895 & 6.519414 & 677.87 \\
\hline $\mathbf{1 1 5 0}$ & $\mathbf{?}$ & $\mathbf{4 . 6 9 4 9 1 2}$ & $\boldsymbol{?}$ & $\mathbf{1 0 9 . 3 9}$ \\
\hline &. & & & \\
\hline
\end{tabular}




\section{HM (Crucible and Spindle Set B)} 4/28/99

\begin{tabular}{lrr}
\multicolumn{3}{c}{ JMP® Nonlinear Regression } \\
Parameter & Estimate & ApproxStdErr \\
A & -4.320142575 & 0.41438758 \\
B & 9711.6078471 & 823.40395 \\
C & 84.219234228 & 41.9154646
\end{tabular}

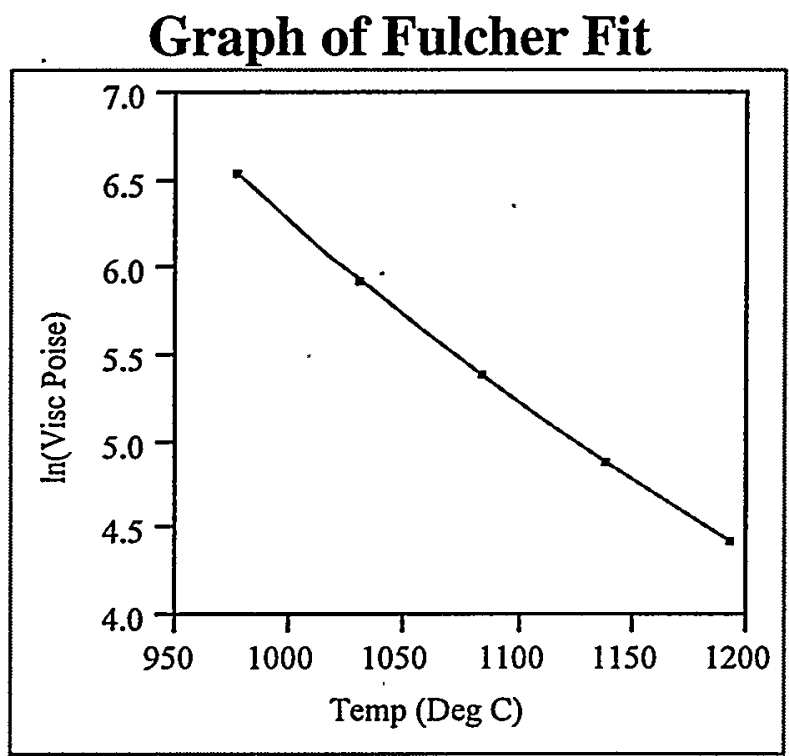

HM

\begin{tabular}{|c|c|c|c|c|}
\hline $\begin{array}{c}\text { Temp } \\
\text { (Deg C) }\end{array}$ & $\begin{array}{c}\text { Measured } \\
\text { Visc. } \\
\text { (Poise) }\end{array}$ & $\begin{array}{c}\text { In Visc } \\
\text { (Fulcher, } \\
\text { Poise) }\end{array}$ & $\begin{array}{c}\text { Measured } \\
\text { InVisc. } \\
\text { (Poise) }\end{array}$ & $\begin{array}{c}\text { Predicted } \\
\text { Visc. } \\
\text { (poise) }\end{array}$ \\
\hline 1193 & 84.45807 & 4.438674 & 4.436255 & 84.66 \\
\hline 1138.5 & 133.8322 & 4.891453 & 4.896587 & 133.15 \\
\hline 1083.5 & 220.617 & 5.398455 & 5.396428 & 221.06 \\
\hline 1030.5 & 380.3218 & 5.942782 & 5.941018 & 380.99 \\
\hline 977.5 & 701.1882 & 6.5517 & 6.552776 & 700.43 \\
\hline 1150 & $\mathbf{?}$ & $\mathbf{4 . 7 9 2 0 5 8}$ & $\mathbf{?}$ & $\mathbf{1 2 0 . 5 5}$ \\
\hline & $\mathbf{r}$ & & & \\
\hline
\end{tabular}




\section{Batch 1 (Crucible and Spindle Set B) 4/30/99}

$\begin{array}{lrr}\text { JMP® } & \text { Nonlinear Regression } \\ \text { Parameter } & \begin{array}{r}\text { Estimate } \\ \text { ApproxStdErr }\end{array} \\ \text { A } & -4.874851565 & 0.5159502 \\ \text { B } & 9491.1788828 & 1051.02319 \\ \text { C } & 60.500972194 & 56.1382161\end{array}$

Graph of Fulcher Fit

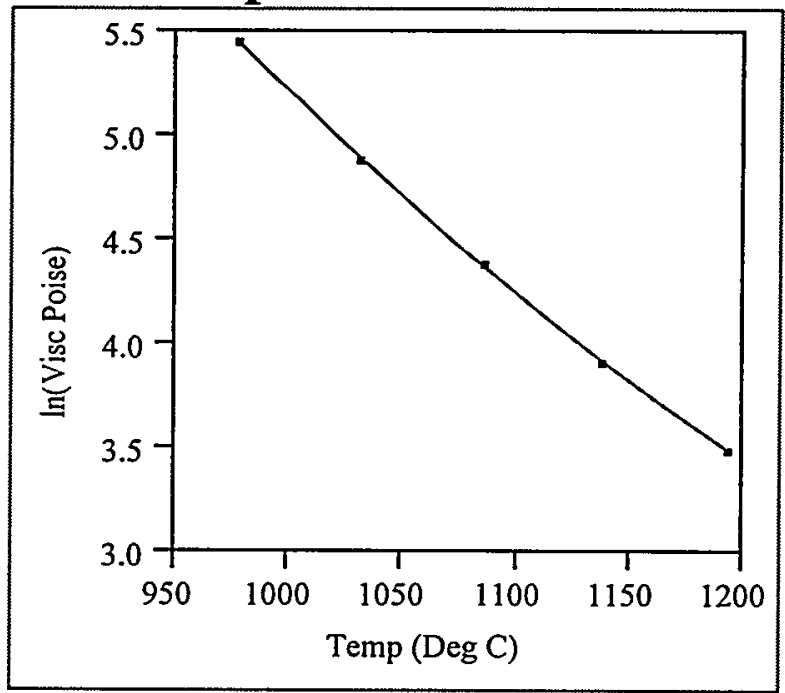

Batch 1

\begin{tabular}{|c|c|c|c|c|}
\hline $\begin{array}{c}\text { Temp } \\
\text { (Deg C) }\end{array}$ & $\begin{array}{c}\text { Measured } \\
\text { Visc. } \\
\text { (Poise) }\end{array}$ & $\begin{array}{c}\text { In Visc } \\
\text { (Fulcher, } \\
\text { Poise) }\end{array}$ & $\begin{array}{c}\text { Measured } \\
\text { InVisc. } \\
\text { (Poise) }\end{array}$ & $\begin{array}{c}\text { Predicted } \\
\text { Visc. } \\
\text { (poise) }\end{array}$ \\
\hline 1194 & 33.1117 & 3.498494 & 3.499887 & 33.07 \\
\hline 1139 & 50.45036 & 3.925508 & 3.92099 & 50.68 \\
\hline 1086 & 80.28315 & 4.380329 & 4.38556 & 79.86 \\
\hline 1032 & 133.2478 & 4.894771 & 4.89221 & 133.59 \\
\hline 979 & 234.8533 & 5.458506 & 5.458961 & 234.75 \\
\hline $\mathbf{1 1 5 0}$ & $\mathbf{?}$ & $\mathbf{3 . 8 3 6 6 5 6}$ & $\mathbf{?}$ & $\mathbf{4 6 . 3 7}$ \\
\hline &. & & & \\
\hline
\end{tabular}




\section{Glass 1}

5/4/99

\begin{tabular}{lrr}
\multicolumn{3}{c}{ JMP ${ }^{\circledR}$ Nonlinear Regression } \\
Parameter & Estimate & ApproxStdErr \\
A & -4.699833614 & 0.76175876 \\
B & 9562.1367256 & 1483.96856 \\
C & 107.18914576 & 75.2170732
\end{tabular}

Graph of Fulcher Fit

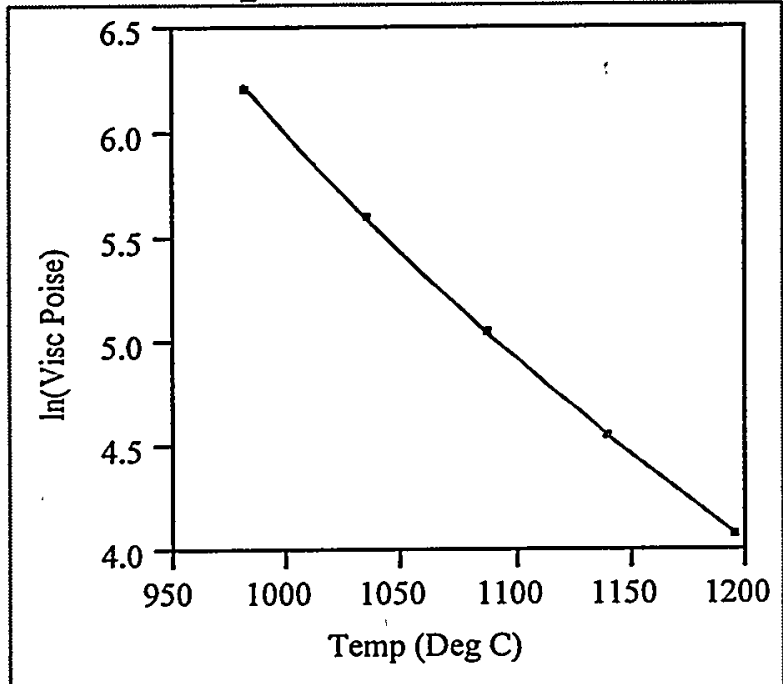

Glass 1

\begin{tabular}{|c|c|c|c|c|}
\hline $\begin{array}{c}\text { Temp } \\
\text { (Deg C) }\end{array}$ & $\begin{array}{c}\text { Measured } \\
\text { Visc. } \\
\text { (Poise) }\end{array}$ & $\begin{array}{c}\text { In Visc } \\
\text { (Fulcher, } \\
\text { Poise) }\end{array}$ & $\begin{array}{c}\text { Measured } \\
\text { InVisc. } \\
\text { (Poise) }\end{array}$ & $\begin{array}{c}\text { Predicted } \\
\text { Visc. } \\
\text { (poise) }\end{array}$ \\
\hline 1195.5 & 59.73861 & 4.086385 & 4.089979 & 59.52 \\
\hline 1139.5 & 94.99094 & 4.563013 & 4.553782 & 95.87 \\
\hline 1088 & 156.9593 & 5.049382 & 5.055986 & 155.93 \\
\hline 1035 & 272.0865 & 5.606294 & 5.60612 & 272.13 \\
\hline 982.5 & 504.5407 & 6.224442 & 6.223648 & 504.94 \\
\hline $\mathbf{1 1 5 0}$ & $\mathbf{?}$ & $\mathbf{4 . 4 6 9 7 4 6}$ & $\mathbf{?}$ & $\mathbf{8 7 . 3 3}$ \\
\hline & - & & & \\
\hline
\end{tabular}




\section{Purex \\ $5 / 7 / 99$}

\begin{tabular}{lrr}
\multicolumn{2}{c}{ JMP® ${ }^{\text {Nonlinear }}$ Regression } \\
Parameter & $\begin{array}{r}\text { Estimate } \\
\text { ApproxStdErr }\end{array}$ \\
A & -4.372307534 & 0.15629799 \\
B & 7485.1219273 & 293.275854 \\
C & 143.34920218 & 18.2825477
\end{tabular}

Graph of Fulcher Fit

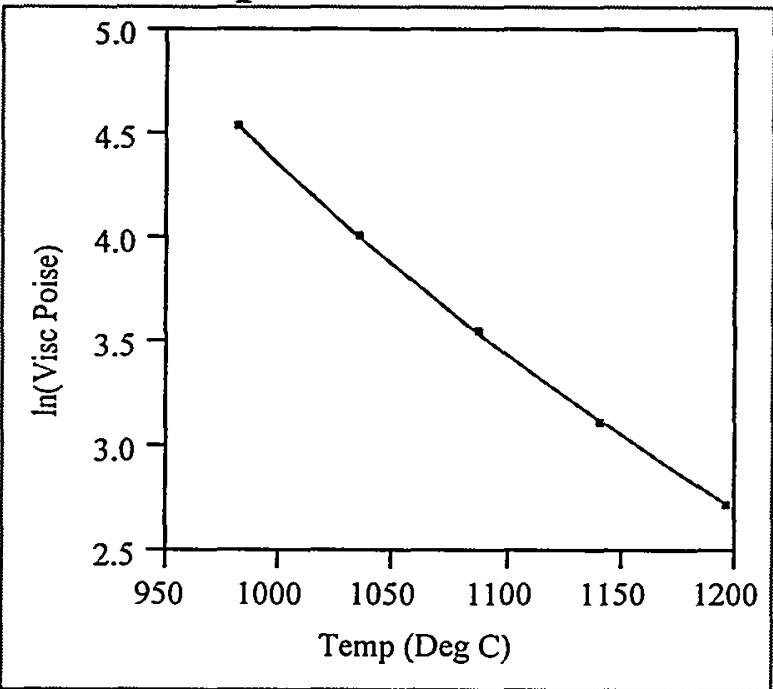

Purex

\begin{tabular}{|c|c|c|c|c|}
\hline $\begin{array}{c}\text { Temp } \\
\text { (Deg C) }\end{array}$ & $\begin{array}{c}\text { Measured } \\
\text { Visc. } \\
\text { (Poise) }\end{array}$ & $\begin{array}{c}\text { In Visc } \\
\text { (Fulcher, } \\
\text { Poise) }\end{array}$ & $\begin{array}{c}\text { Measured } \\
\text { InVisc. } \\
\text { (Poise) }\end{array}$ & $\begin{array}{c}\text { Predicted } \\
\text { Visc. } \\
\text { (poise) }\end{array}$ \\
\hline 1196.5 & 15.41173 & 2.735053 & 2.735129 & 15.41 \\
\hline 1141.5 & 22.77908 & 3.126682 & 3.125843 & 22.80 \\
\hline 1088 & 34.92775 & 3.551384 & 3.553282 & 34.86 \\
\hline 1035 & 55.74455 & 4.022371 & 4.02078 & 55.83 \\
\hline 982.5 & 94.44585 & 4.547569 & 4.548027 & 94.40 \\
\hline $\mathbf{1 1 5 0}$ & $\mathbf{?}$ & $\mathbf{3 . 0 6 3 3 6 1}$ & $\mathbf{?}$ & $\mathbf{2 1 . 4 0}$ \\
\hline & & & & \\
\hline
\end{tabular}




\section{Glass 3}

5/11/99

\section{JMP® Nonlinear Regression Parameter Estimate . ApproxStdErr \\ A \\ B \\ C \\ $-3.949730409$ \\ 8562.9111795 \\ 150.96686754 \\ 0.61395649 \\ 1141.73437 \\ 61.6522803}

Graph of Fulcher Fit

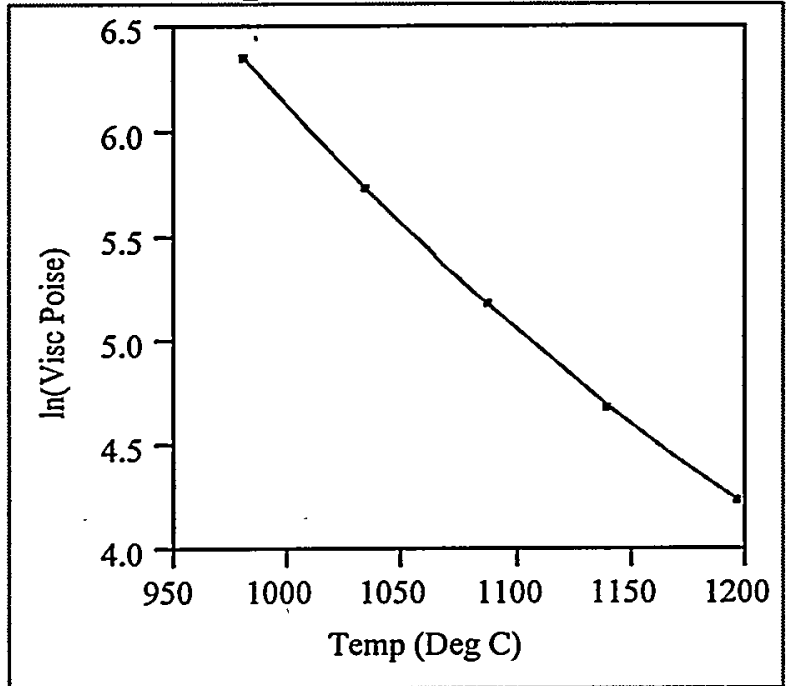

Glass 3

\begin{tabular}{|c|c|c|c|c|}
\hline $\begin{array}{c}\text { Temp } \\
\text { (Deg C) }\end{array}$ & $\begin{array}{c}\text { Measured } \\
\text { Visc. } \\
\text { (Poise) }\end{array}$ & $\begin{array}{c}\text { In Visc } \\
\text { (Fulcher, } \\
\text { Poise) }\end{array}$ & $\begin{array}{c}\text { Measured } \\
\text { InVisc. } \\
\text { (Poise) }\end{array}$ & $\begin{array}{c}\text { Predicted } \\
\text { Visc. } \\
\text { (poise) }\end{array}$ \\
\hline 1196.5 & 69.60793 & 4.240265 & 4.242878 & 69.43 \\
\hline 1140.5 & 109.5417 & 4.703756 & 4.696305 & 110.36 \\
\hline 1088 & 180.4601 & 5.188592 & 5.195509 & 179.22 \\
\hline 1034 & 312.7171 & 5.747427 & 5.745299 & 313.38 \\
\hline 981.5 & 578.5096 & 6.360408 & 6.360455 & 578.48 \\
\hline $\mathbf{1 1 5 0}$ & $\mathbf{?}$ & $\mathbf{4 . 6 2 1 4 6 8}$ & $\mathbf{?}$ & $\mathbf{1 0 1 . 6 4}$ \\
\hline & - & & & \\
\hline
\end{tabular}




\section{Batch 1}

6/8/99

$\begin{array}{lrr}\text { Parameter } & \begin{array}{r}\text { JMP® } \\ \text { Nonlinear Regression }\end{array} \\ \text { Estimate } & \text { Regrer } \\ \text { ApproxStdErr }\end{array}$

Graph of Fulcher Fit

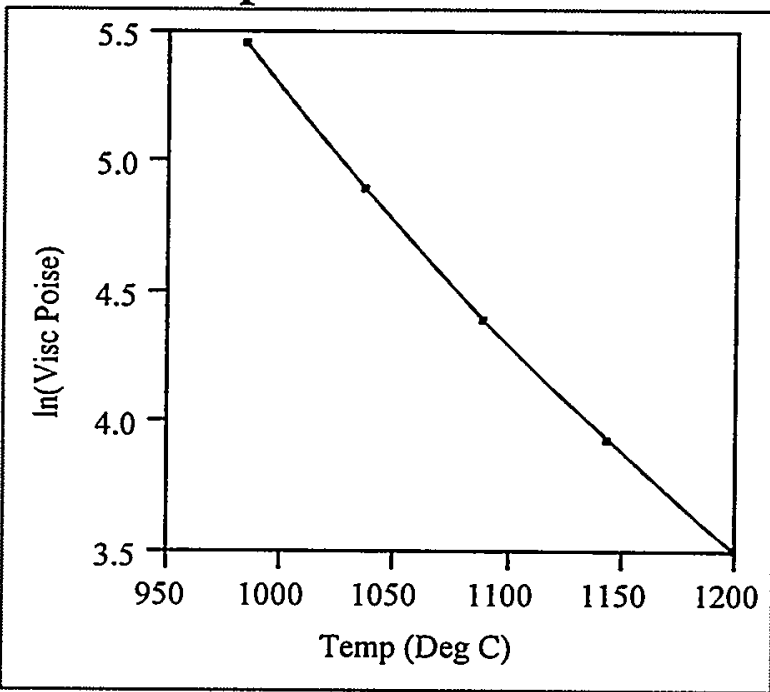

Batch 1

\begin{tabular}{|c|c|c|c|c|}
\hline $\begin{array}{c}\text { Temp } \\
\text { (Deg C) }\end{array}$ & $\begin{array}{c}\text { Measured } \\
\text { Visc. } \\
\text { (Poise) }\end{array}$ & $\begin{array}{c}\text { In Visc } \\
\text { (Fulcher, } \\
\text { Poise) }\end{array}$ & $\begin{array}{c}\text { Measured } \\
\text { InVisc. } \\
\text { (Poise) }\end{array}$ & $\begin{array}{c}\text { Predicted } \\
\text { Visc. } \\
\text { (poise) }\end{array}$ \\
\hline 1199.5 & 33.6289 & 3.514689 & 3.515386 & 33.61 \\
\hline 1143.5 & 51.48968 & 3.941739 & 3.941381 & 51.51 \\
\hline 1089.5 & 81.3304 & 4.401077 & 4.39852 & 81.54 \\
\hline 1037 & 134.8082 & 4.90048 & 4.903853 & 134.35 \\
\hline 984 & 236.5726 & 5.46741 & 5.466255 & 236.85 \\
\hline $\mathbf{1 1 5 0}$ & $\mathbf{?}$ & $\mathbf{3 . 8 8 9 7 5}$ & $\mathbf{?}$ & $\mathbf{4 8 . 9 0}$ \\
\hline & & & & \\
\hline
\end{tabular}




\section{Batch 1 (Crucible and Spindle Set B) 6/9/99}

\begin{tabular}{lrr}
\multicolumn{3}{c}{ JMP® ${ }^{\circledR}$ Nonlinear Regression } \\
Parameter & Estimate & ApproxStdErr \\
A & -3.478220023 & 0.37284869 \\
B & 6922.5011794 & 652.766354 \\
C & 206.14511702 & 41.019357
\end{tabular}

Graph of Fulcher Fit

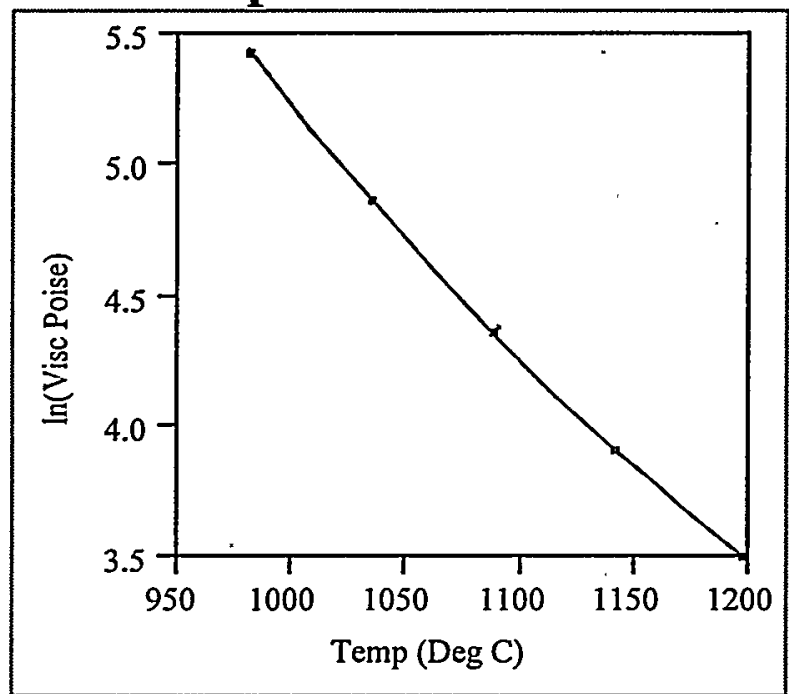

Batch 1

\begin{tabular}{|c|c|c|c|c|}
\hline $\begin{array}{c}\text { Temp } \\
\text { (Deg C) }\end{array}$ & $\begin{array}{c}\text { Measured } \\
\text { Visc. } \\
\text { (Poise) }\end{array}$ & $\begin{array}{c}\text { In Visc } \\
\text { (Fulcher, } \\
\text { Poise) }\end{array}$ & $\begin{array}{c}\text { Measured } \\
\text { InVisc. } \\
\text { (Poise) }\end{array}$ & $\begin{array}{c}\text { Predicted } \\
\text { Visc. } \\
\text { (poise) }\end{array}$ \\
\hline 1198 & 33.24714 & 3.501129 & 3.503969 & 33.15 \\
\hline 1142 & 50.05937 & 3.918761 & 3.91321 & 50.34 \\
\hline 1089 & 78.5489 & 4.362821 & 4.363721 & 78.48 \\
\hline 1035.5 & 130.5924 & 4.86863 & 4.872081 & 130.14 \\
\hline 982.5 & 229.7086 & 5.438451 & 5.436812 & 230.09 \\
\hline $\mathbf{1 1 5 0}$ & $\mathbf{?}$ & $\mathbf{3 . 8 5 6 0 6 5}$ & $\mathbf{?}$ & $\mathbf{4 7 . 2 8}$ \\
\hline & $\mathbf{2}$ & & & \\
\hline
\end{tabular}




\section{Batch 1} 8/19/99

\section{JMP® Nonlinear Regression Parameter Estimate . ApproxStdErr A $-4.546871487$ 0.20916011 \\ B 8960.434251 \\ 415.591051 \\ C \\ 90.405311289 \\ 22.929557}

Graph of Fulcher Fit

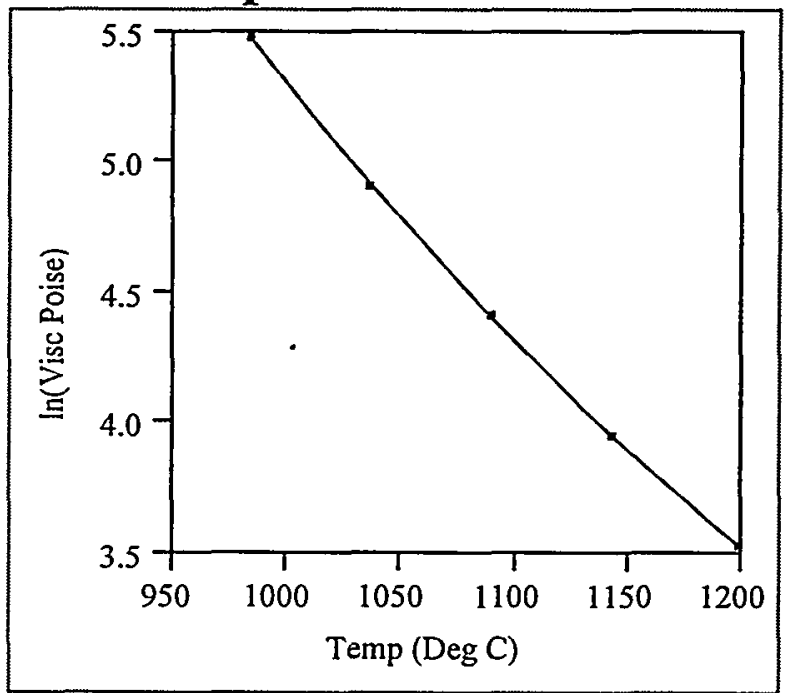

Batch 1

\begin{tabular}{|c|c|c|c|c|}
\hline $\begin{array}{c}\text { Temp } \\
\text { (Deg C) }\end{array}$ & $\begin{array}{c}\text { Measured } \\
\text { Visc. } \\
\text { (Poise) }\end{array}$ & $\begin{array}{c}\text { In Visc } \\
\text { (Fulcher, } \\
\text { Poise) }\end{array}$ & $\begin{array}{c}\text { Measured } \\
\text { InVisc. } \\
\text { (Poise) }\end{array}$ & $\begin{array}{c}\text { Predicted } \\
\text { Visc. } \\
\text { (poise) }\end{array}$ \\
\hline 1199 & 34.35115 & 3.535825 & 3.536636 & 34.32 \\
\hline 1143.5 & 52.43348 & 3.961798 & 3.959545 & 52.55 \\
\hline 1090 & 83.03358 & 4.417196 & 4.419245 & 82.86 \\
\hline 1037 & 136.7972 & 4.919096 & 4.918499 & 136.88 \\
\hline 984 & 239.9716 & 5.480532 & 5.480521 & 239.97 \\
\hline $\mathbf{1 1 5 0}$ & $\mathbf{?}$ & $\mathbf{3 . 9 0 9 6 0 2}$ & $\mathbf{?}$ & $\mathbf{4 9 . 8 8}$ \\
\hline & - & & & \\
\hline
\end{tabular}




\section{Batch 1 (Crucible and Spindle Set B) 8/31/99}

\begin{tabular}{lrr}
\multicolumn{3}{c}{ JMP® Nonlinear Regression } \\
Parameter & Estimate & ApproxStdErr \\
A & -4.429398801 & 0.82998878 \\
B & 8734.1932506 & 1633.32405 \\
C & 101.72050786 & 91.5526932
\end{tabular}

Graph of Fulcher Fit

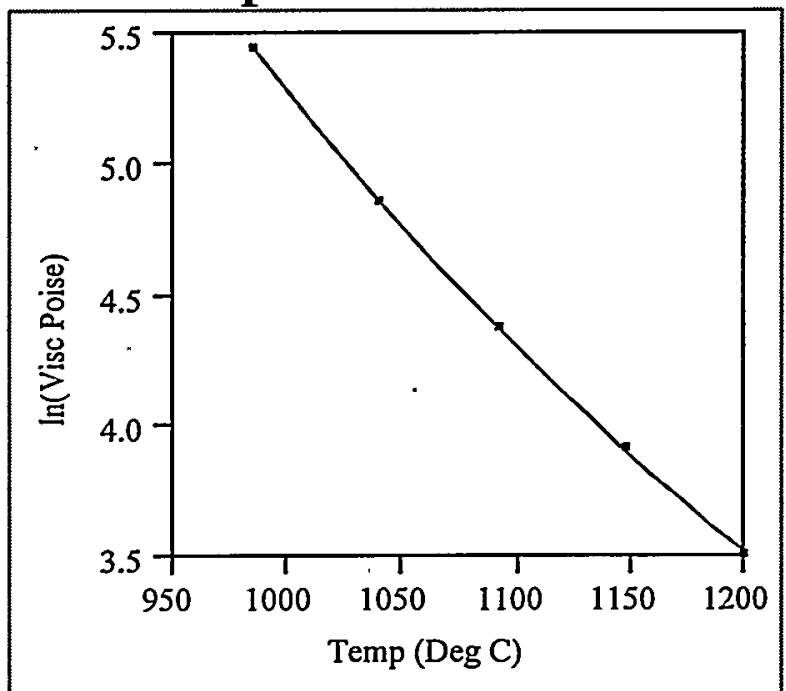

Batch 1

\begin{tabular}{|c|c|c|c|c|}
\hline $\begin{array}{c}\text { Temp } \\
\text { (Deg C) }\end{array}$ & $\begin{array}{c}\text { Measured } \\
\text { Visc. } \\
\text { (Poise) }\end{array}$ & $\begin{array}{c}\text { In Visc } \\
\text { (Fulcher, } \\
\text { Poise) }\end{array}$ & $\begin{array}{c}\text { Measured } \\
\text { InVisc. } \\
\text { (Poise) }\end{array}$ & $\begin{array}{c}\text { Predicted } \\
\text { Visc. } \\
\text { (poise) }\end{array}$ \\
\hline 1200 & 33.72206 & 3.523216 & 3.518152 & 33.89 \\
\hline 1148 & 50.84697 & 3.91846 & 3.928821 & 50.32 \\
\hline 1092 & 80.36987 & 4.390529 & 4.386639 & 80.68 \\
\hline 1040 & 131.0838 & 4.879334 & 4.875837 & 131.54 \\
\hline 986 & 232.7295 & 5.447787 & 5.449877 & 232.24 \\
\hline $\mathbf{1 1 5 0}$ & $\mathbf{?}$ & $\mathbf{3 . 9 0 2 5 3 3}$ & $\mathbf{?}$ & $\mathbf{4 9 . 5 3}$ \\
\hline & - & & & \\
\hline
\end{tabular}




\section{Uranium Standard Glass 10/8/99}

\begin{tabular}{lrr}
\multicolumn{3}{c}{ JMP® Nonlinear Regression } \\
Parameter & Estimate & ApproxStdErr \\
A & -3.565831384 & 0.24348389 \\
B & 6350.8231927 & 406.90651 \\
C & 247.41179886 & 26.5893295
\end{tabular}

Graph of Fulcher Fit

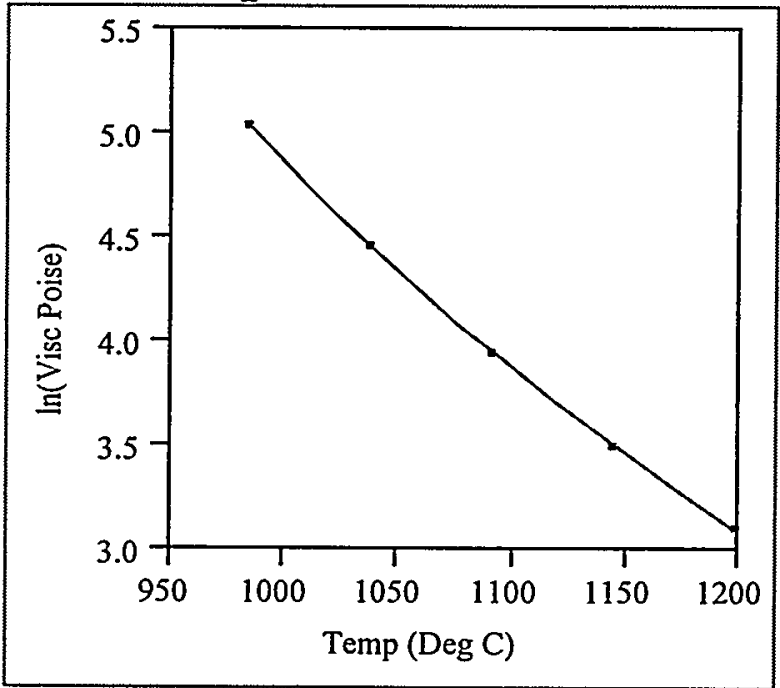

Uranium Standard Glass

\begin{tabular}{|c|c|c|c|c|}
\hline $\begin{array}{c}\text { Temp } \\
\text { (Deg C) }\end{array}$ & $\begin{array}{c}\text { Measured } \\
\text { Visc. } \\
\text { (Poise) }\end{array}$ & $\begin{array}{c}\text { In Visc } \\
\text { (Fulcher, } \\
\text { Poise) }\end{array}$ & $\begin{array}{c}\text { Measured } \\
\text { InVisc. } \\
\text { (Poise) }\end{array}$ & $\begin{array}{c}\text { Predicted } \\
\text { Visc. } \\
\text { (poise) }\end{array}$ \\
\hline 1199.5 & 22.31736418 & 3.104583 & 3.105365 & 22.30 \\
\hline 1145 & 33.42725769 & 3.509599 & 3.509372 & 33.43 \\
\hline 1091 & 52.4221759 & 3.962514 & 3.95933 & 52.59 \\
\hline 1038.5 & 87.01031712 & 4.462127 & 4.466027 & 86.67 \\
\hline 984.5 & 155.8658691 & 5.050265 & 5.048996 & 156.06 \\
\hline $\mathbf{1 1 5 0}$ & $\mathbf{?}$ & $\mathbf{3 . 4 7 0 4 0 4}$ & $\mathbf{?}$ & $\mathbf{3 2 . 1 5}$ \\
\hline &. & & & \\
\hline
\end{tabular}




\section{PNNL}

HM

$\begin{array}{lrr}\text { Parameter } & \text { Estimate } & \text { ApproxStdErr } \\ \text { A } & -2.193010134 & 0.32322725 \\ \text { B } & 5907.120649 & 556.112826 \\ \text { C } & 305.22809368 & 40.17480\end{array}$

JMP ${ }^{\circledR}$ Nonlinear Regression

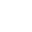

Graph of Fulcher Fit

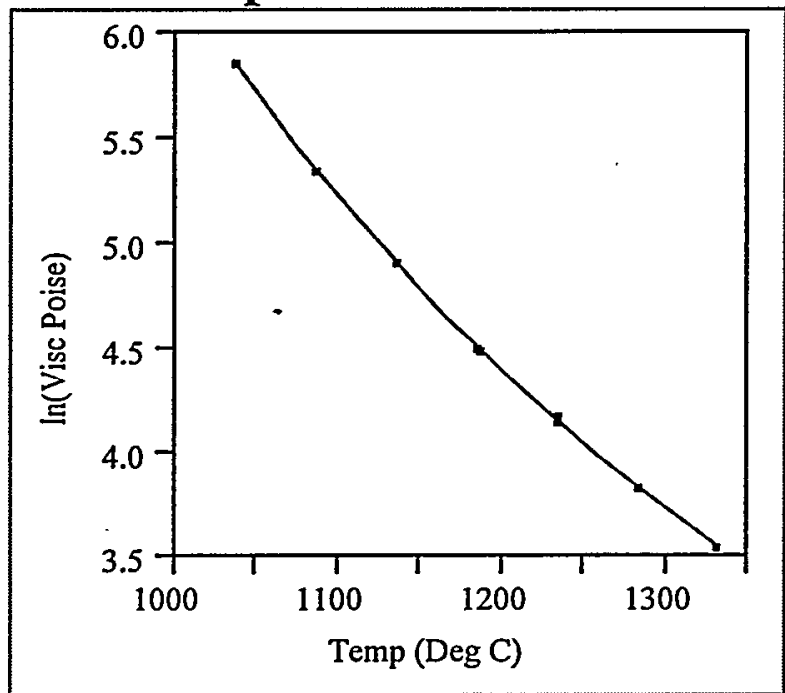

HM Measured by PNNL

\begin{tabular}{|c|c|c|c|c|}
\hline $\begin{array}{c}\text { Temp } \\
(\operatorname{Deg} C)\end{array}$ & $\begin{array}{c}\text { Measured } \\
\text { Visc. } \\
\text { (Poise) }\end{array}$ & $\begin{array}{c}\text { In Visc } \\
\text { (Fulcher, } \\
\text { Poise) }\end{array}$ & $\begin{array}{l}\text { Measured } \\
\text { InVisc. } \\
\text { (Poise) }\end{array}$ & $\begin{array}{c}\text { Predicted } \\
\text { Visc. } \\
\text { (poise) }\end{array}$ \\
\hline 1235 & 65.13 & 4.160291 & 4.176385 & 64.09 \\
\hline 1187 & 89.54 & 4.506138 & 4.494685 & 90.57 \\
\hline 1137 & 136.34 & 4.908841 & 4.915152 & 135.48 \\
\hline 1186 & 90.46 & 4.513744 & 4.504908 & 91.26 \\
\hline 1235 & 64.48 & 4.160291 & 4.166355 & 64.09 \\
\hline 1284 & 46.52 & 3.842227 & 3.839882 & 46.63 \\
\hline 1333 & 34.75 & 3.554491 & 3.54818 & 34.97 \\
\hline 1236 & 63.93 & 4.153465 & 4.157789 & 63.65 \\
\hline 1088 & 209.51 & 5.353404 & 5.344771 & 211.33 \\
\hline 1039 & 351.47 & 5.85734 & 5.862124 & 349.79 \\
\hline 1150 & $?$ & 4.799553 & $?$ & 121.46 \\
\hline
\end{tabular}




\section{PNNL}

\section{Glass 1}

\begin{tabular}{lrr}
\multicolumn{3}{c}{ JMP $\AA$ Nonlinear Regression } \\
Parameter & $\begin{array}{r}\text { Estimate } \\
\text { ApproxStdErr }\end{array}$ \\
A & -6.454877277 & 1.43078068 \\
B & 13955.979894 & 3567.35447 \\
C & -125.5193544 & 158.757186
\end{tabular}

Graph of Fulcher Fit

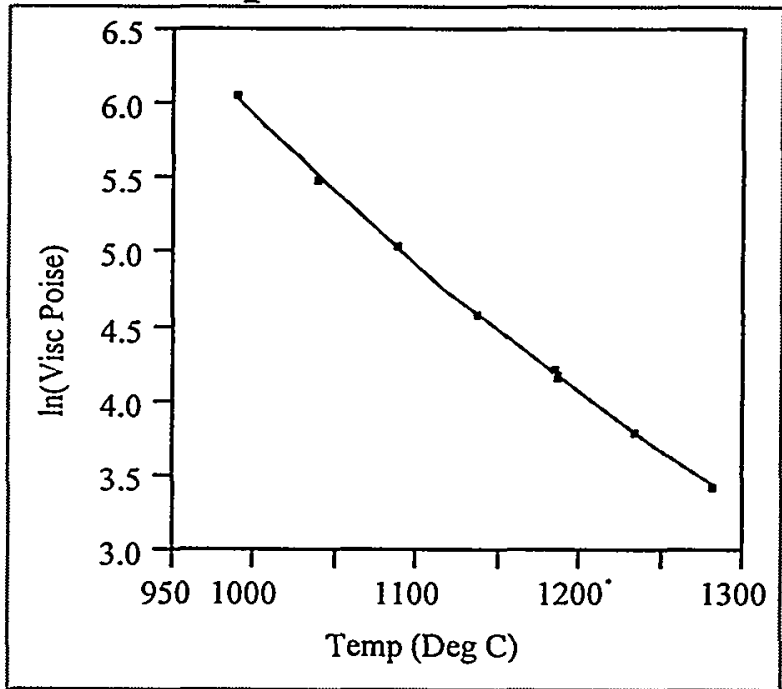

Glass 1 Measured by PNNL

\begin{tabular}{|c|c|c|c|c|}
\hline $\begin{array}{c}\text { Temp } \\
\text { (Deg C) }\end{array}$ & $\begin{array}{c}\text { Measured } \\
\text { Visc. } \\
\text { (Poise) }\end{array}$ & $\begin{array}{c}\text { In Visc } \\
\text { (Fulcher, } \\
\text { Poise) }\end{array}$ & $\begin{array}{c}\text { Measured } \\
\text { InVisc. } \\
\text { (Poise) }\end{array}$ & $\begin{array}{c}\text { Predicted } \\
\text { Visc. } \\
\text { (poise) }\end{array}$ \\
\hline 1186 & 67.98 & 4.186201 & 4.219214 & 65.77 \\
\hline 1138 & 98.01 & 4.590446 & 4.58507 & 98.54 \\
\hline 1088 & 156.05 & 5.045541 & 5.050176 & 155.33 \\
\hline 1137 & 98.38 & 4.599195 & 4.588838 & 99.40 \\
\hline 1187 & 66.42 & 4.178093 & 4.195998 & 65.24 \\
\hline 1235 & 44.58 & 3.802955 & 3.797285 & 44.83 \\
\hline 1283 & 31.21 & 3.453386 & 3.440739 & 31.61 \\
\hline 1187 & 64.76 & 4.178093 & 4.170688 & 65.24 \\
\hline 1039 & 243.78 & 5.529449 & 5.496266 & 252.01 \\
\hline 990 & 434.83 & 6.055869 & 6.074955 & 426.61 \\
\hline $\mathbf{1 1 5 0}$ & $-\mathbf{?}$ & $\mathbf{4 . 4 8 6 5 3 2}$ & $\mathbf{?}$ & $\mathbf{8 8 . 8 1}$ \\
\hline & & & & \\
\hline
\end{tabular}


PNNL

Glass 3

JMP ${ }^{\circledR}$ Nonlinear Regression

$\begin{array}{lrr}\text { Parameter } & \text { Estimate } & \text { ApproxStdErr } \\ \text { A } & -4.806614893 & 0.76767238 \\ \text { B } & 10310.952765 & 1640.15538 \\ \text { C } & 49.452807306 & 84.5435612\end{array}$

Graph of Fulcher Fit

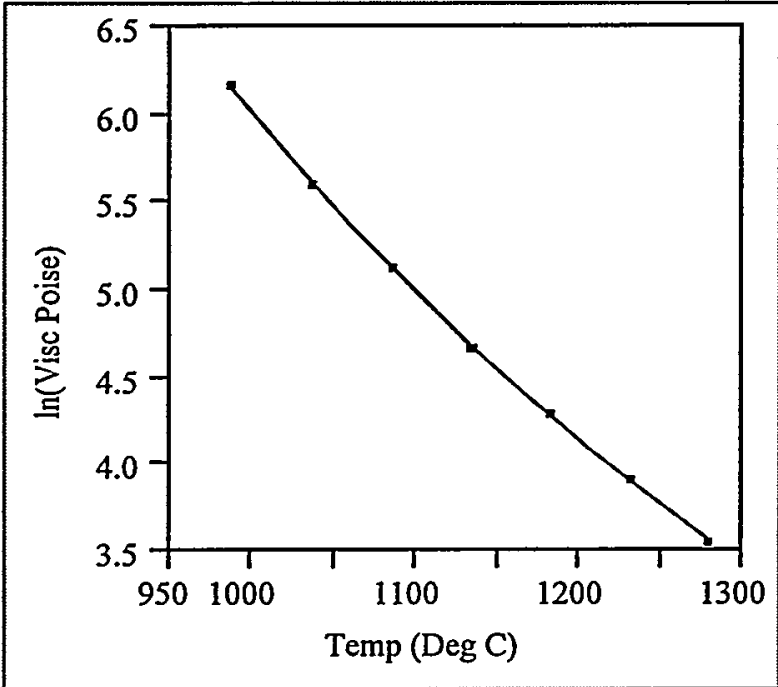

Glass 3 Measured by PNNL

\begin{tabular}{|c|c|c|c|c|}
\hline $\begin{array}{c}\text { Temp } \\
\text { (Deg C) }\end{array}$ & $\begin{array}{c}\text { Measured } \\
\text { Visc. } \\
\text { (Poise) }\end{array}$ & $\begin{array}{c}\text { In Visc } \\
\text { (Fulcher, } \\
\text { Poise) }\end{array}$ & $\begin{array}{l}\text { Measured } \\
\text { InVisc. } \\
\text { (Poise) }\end{array}$ & $\begin{array}{c}\text { Predicted } \\
\text { Visc. } \\
\text { (poise) }\end{array}$ \\
\hline 1184 & 73.32 & 4.281551 & 4.294833 & 72.35 \\
\hline 1136 & 107.22 & 4.683035 & 4.674883 & 108.10 \\
\hline 1087 & 170.23 & 5.131201 & 5.13715 & 169.22 \\
\hline 1135 & 107.59 & 4.691777 & 4.678328 & 109.05 \\
\hline 1184 & 72.96 & 4.281551 & 4.289911 & 72.35 \\
\hline 1233 & 50.2 & 3.905292 & 3.916015 & 49.66 \\
\hline 1281 & 34.75 & 3.565742 & 3.54818 & 35.37 \\
\hline 1184 & 73.05 & 4.281551 & 4.291144 & 72.35 \\
\hline 1037 & 273.81 & 5.634357 & 5.612434 & 279.88 \\
\hline 989 & 483.45 & 6.16777 & 6.180948 & 477.12 \\
\hline 1150 & $-\quad ?$ & 4.562318 & ? & 95.81 \\
\hline
\end{tabular}




\section{PNNL}

\section{Glass 5}

\begin{tabular}{lrr}
\multicolumn{3}{c}{ JMP® ${ }^{\circledR}$ Nonlinear Regression } \\
Parameter & $\begin{array}{r}\text { Estimate } \\
\text { ApproxStdErr }\end{array}$ \\
A & -4.194028723 & 0.46612412 \\
B & 9166.6082047 & 935.617028 \\
C & 113.63583061 & 50.9315169
\end{tabular}

Graph of Fulcher Fit

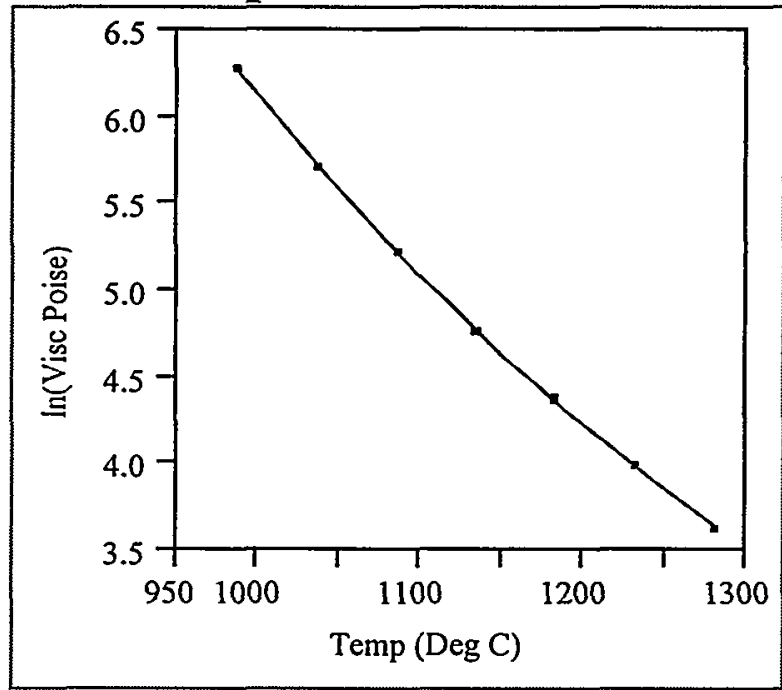

Glass 5 Measured by PNNL

\begin{tabular}{|c|c|c|c|c|}
\hline $\begin{array}{c}\text { Temp } \\
\text { (Deg C) }\end{array}$ & $\begin{array}{c}\text { Measured } \\
\text { Visc. } \\
\text { (Poise) }\end{array}$ & $\begin{array}{c}\text { In Visc } \\
\text { (Fulcher, } \\
\text { Poise) }\end{array}$ & $\begin{array}{c}\text { Measured } \\
\text { InVisc. } \\
\text { (Poise) }\end{array}$ & $\begin{array}{c}\text { Predicted } \\
\text { Visc. } \\
\text { (poise) }\end{array}$ \\
\hline 1184 & 80.23 & 4.36998 & 4.384898 & 79.04 \\
\hline 1136 & 117.17 & 4.77206 & 4.763626 & 118.16 \\
\hline 1087 & 185.34 & 5.223421 & 5.222192 & 185.57 \\
\hline 1135 & 117.91 & 4.780839 & 4.769922 & 119.20 \\
\hline 1184 & 79.5 & 4.36998 & 4.375757 & 79.04 \\
\hline 1233 & 54.35 & 3.995092 & 3.995445 & 54.33 \\
\hline 1282 & 38.14 & 3.651649 & 3.641264 & 38.54 \\
\hline 1184 & 79.96 & 4.36998 & 4.381527 & 79.04 \\
\hline 1038 & 303.13 & 5.722635 & 5.714162 & 305.71 \\
\hline 989 & 536.24 & 6.277737 & 6.284582 & 532.58 \\
\hline $\mathbf{1 1 5 0}$ & $\mathbf{P}$ & $\mathbf{4 . 6 5 0 9 4}$ & $\mathbf{?}$ & $\mathbf{1 0 4 . 6 8}$ \\
\hline & & & & \\
\hline
\end{tabular}




\section{CELS}

HIM

$\mathrm{JMP} \circledast$ Nonlinear Regression

$\begin{array}{lrr}\text { Parameter } & \text { Estimate } & \text { ApproxStdErr } \\ \text { A } & -2.483958723 & 0.11834895 \\ \text { B } & 6343.3354217 & 133.842921 \\ \text { C } & 268.78934915 & 5.59577481\end{array}$

Graph of Fulcher Fit

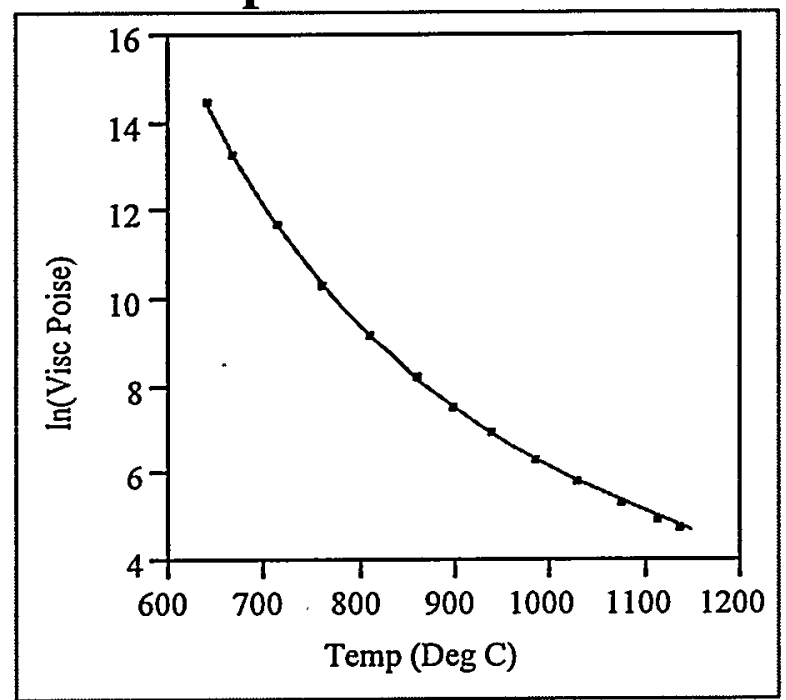

HM Measured by CELS

\begin{tabular}{|c|c|c|c|c|}
\hline $\begin{array}{c}\text { Temp } \\
\text { (Deg C) }\end{array}$ & $\begin{array}{c}\text { Measured } \\
\text { Visc. } \\
\text { (Poise) }\end{array}$ & $\begin{array}{c}\text { In Visc } \\
\text { (Fulcher, } \\
\text { Poise) }\end{array}$ & $\begin{array}{c}\text { Measured } \\
\text { InVisc. } \\
\text { (Poise) }\end{array}$ & $\begin{array}{c}\text { Predicted } \\
\text { Visc. } \\
\text { (poise) }\end{array}$ \\
\hline 1136.1 & 123.2 & 4.82984 & 4.813809 & 125.19 \\
\hline 1115.2 & 146.6 & 5.010436 & 4.987708 & 149.97 \\
\hline 1075 & 216.2 & 5.384128 & 5.376204 & 217.92 \\
\hline 1029.3 & 349.9 & 5.856931 & 5.857647 & 349.65 \\
\hline 984.8 & 601.2 & 6.375317 & 6.398928 & 587.17 \\
\hline 940.3 & 1096 & 6.962407 & 6.999422 & 1056.17 \\
\hline 899.3 & 1993 & 7.576673 & 7.597396 & 1952.12 \\
\hline 859.3 & 3863 & 8.25816 & 8.259199 & 3858.99 \\
\hline 809.9 & 10310 & 9.238848 & 9.24087 & 10289.18 \\
\hline 762.2 & 31360 & 10.37214 & 10.35329 & 31956.75 \\
\hline 713.6 & 125900 & 11.7768 & 11.74324 & 130195.9 \\
\hline 669 & 622900 & 13.36603 & 13.34214 & 637961.2 \\
\hline 643.2 &. .1975000 & 14.45823 & 14.49608 & 1901643 \\
\hline $\mathbf{1 1 5 0}$ & $\mathbf{?}$ & $\mathbf{4 . 7 1 4 4 7 4}$ & $\mathbf{?}$ & $\mathbf{1 1 1 . 5 5}$ \\
\hline
\end{tabular}




\section{CELS}

Glass 2

\begin{tabular}{lrr}
\multicolumn{3}{c}{ JMP® ${ }^{\text {Nonlinear }}$ Regression } \\
Parameter & Estimate & ApproxStdErr \\
A & -2.753492044 & 0.05972876 \\
B & 5804.0133568 & 62.611818 \\
C & 304.83858554 & 2.63956508
\end{tabular}

Graph of Fulcher Fit

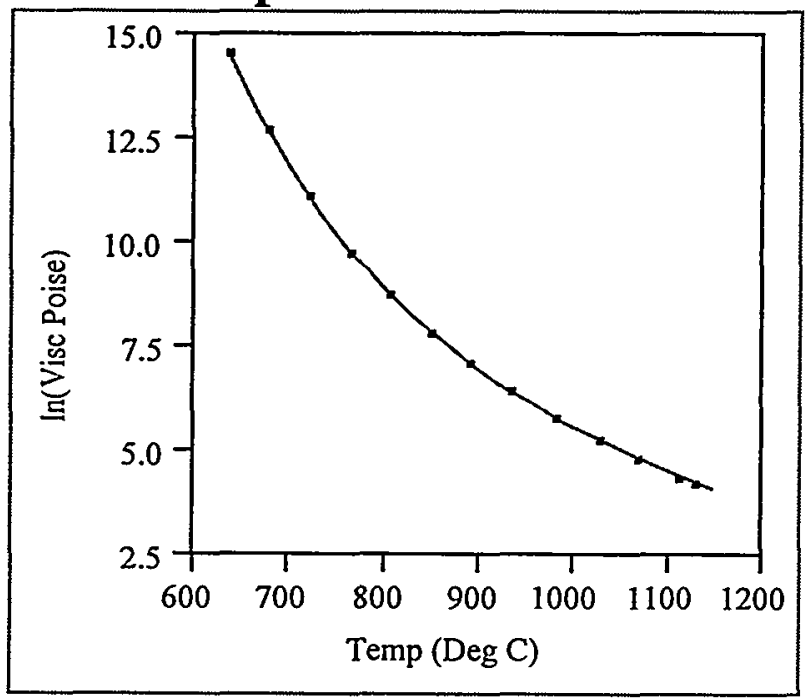

Glass 2 Measured by CELS

\begin{tabular}{|c|c|c|c|c|}
\hline $\begin{array}{c}\text { Temp } \\
\text { (Deg C) }\end{array}$ & $\begin{array}{c}\text { Measured } \\
\text { Visc. } \\
\text { (Poise) }\end{array}$ & $\begin{array}{c}\text { In Visc } \\
\text { (Fulcher, } \\
\text { Poise) }\end{array}$ & $\begin{array}{c}\text { Measured } \\
\text { InVisc. } \\
\text { (Poise) }\end{array}$ & $\begin{array}{c}\text { Predicted } \\
\text { Visc. } \\
\text { (poise) }\end{array}$ \\
\hline 1131.5 & 71.7 & 4.267536 & 4.272491 & 71.35 \\
\hline 1115 & 80.79 & 4.410529 & 4.391853 & 82.31 \\
\hline 1071.5 & 122.8 & 4.817012 & 4.810557 & 123.60 \\
\hline 1028.4 & 196 & 5.26796 & 5.278115 & 194.02 \\
\hline 982.7 & 331 & 5.80875 & 5.802118 & 333.20 \\
\hline 935.7 & 646.5 & 6.446648 & 6.471573 & 630.59 \\
\hline 893.2 & 1244 & 7.111216 & 7.126087 & 1225.64 \\
\hline 851.1 & 2590 & 7.871482 & 7.859413 & 2621.45 \\
\hline 809.5 & 6336 & 8.747315 & 8.754003 & 6293.76 \\
\hline 766.2 & 18060 & 9.826696 & 9.801455 & 18521.66 \\
\hline 723.2 & 67880 & 11.11971 & 11.1255 & 67488.48 \\
\hline 679.8 & 335000 & 12.72547 & 12.72189 & 336202.7 \\
\hline 640.5 & 2070000 & 14.53778 & 14.54306 & 2059104 \\
\hline 1150 & $\mathbf{P}$ & 4.113851 & $\mathbf{2}$ & $\mathbf{6 1 . 1 8}$ \\
\hline
\end{tabular}




\section{CELS}

\section{Glass 4}

\begin{tabular}{|c|c|c|}
\hline \multicolumn{3}{|c|}{ JMP® Nonlinear Regression } \\
\hline Par: & Estimate & ApproxStdErr \\
\hline 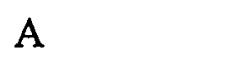 & -2.662146769 & 0.11 \\
\hline & 6132.5283 & \\
\hline & 297.45020649 & \\
\hline
\end{tabular}

Graph of Fulcher Fit

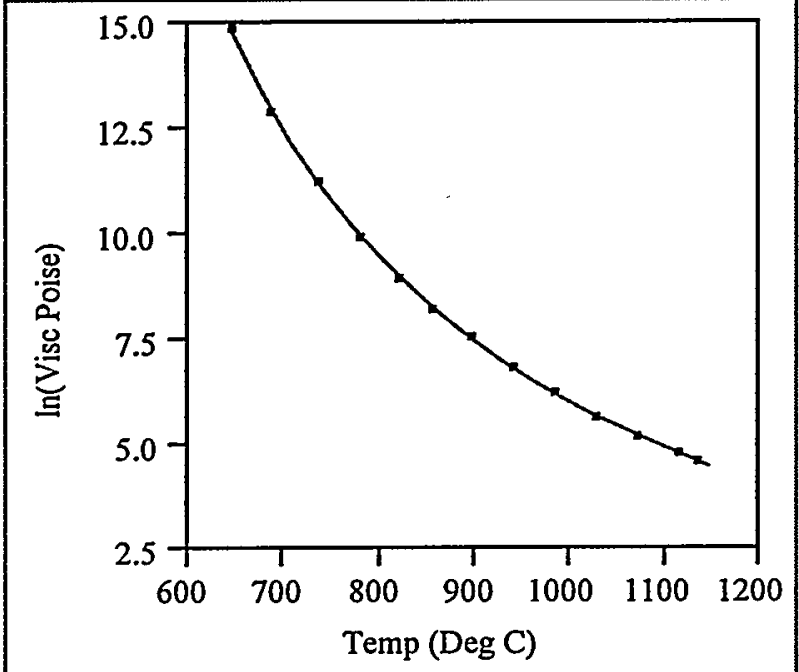

Glass 4 Measured by CELS

\begin{tabular}{|c|c|c|c|c|}
\hline $\begin{array}{c}\text { Temp } \\
\text { (Deg C) }\end{array}$ & $\begin{array}{c}\text { Measured } \\
\text { Visc. } \\
\text { (Poise) }\end{array}$ & $\begin{array}{c}\text { In Visc } \\
\text { (Fulcher, } \\
\text { Poise) }\end{array}$ & $\begin{array}{c}\text { Measured } \\
\text { InVisc. } \\
\text { (Poise) }\end{array}$ & $\begin{array}{c}\text { Predicted } \\
\text { Visc. } \\
\text { (poise) }\end{array}$ \\
\hline 1137.7 & 103.7 & 4.636312 & 4.641502 & 103.16 \\
\hline 1115.6 & 122.8 & 4.833459 & 4.810557 & 125.64 \\
\hline 1074.4 & 182.1 & 5.230935 & 5.204556 & 186.97 \\
\hline 1030.1 & 296.3 & 5.708194 & 5.691372 & 301.33 \\
\hline 985.2 & 531.6 & 6.254655 & 6.275891 & 520.43 \\
\hline 943 & 975.9 & 6.837552 & 6.88336 & 932.20 \\
\hline 898.8 & 1955 & 7.535792 & 7.578145 & 1873.93 \\
\hline 858.3 & 3906 & 8.272204 & 8.270269 & 3913.56 \\
\hline 823.2 & 8076 & 9.002201 & 8.996652 & 8120.94 \\
\hline 781 & 21910 & 10.02016 & 9.994698 & 22475.10 \\
\hline 737.2 & 78140 & 11.28335 & 11.26626 & 79486.92 \\
\hline 690.2 & 411600 & 12.95219 & 12.92781 & 421760.2 \\
\hline 647.6 & 2893000 & 14.85187 & 14.8778 & 2818928 \\
\hline $\mathbf{1 1 5 0}$ & $\mathbf{?}$ & $\mathbf{4 . 5 3 1 0 1 5}$ & $\mathbf{?}$ & $\mathbf{9 2 . 8 5}$ \\
\hline
\end{tabular}




\section{Sharp-Schurtz (SS)}

\section{HM}

\begin{tabular}{|c|c|c|}
\hline \multicolumn{3}{|c|}{ JMP $®$ Nonlinear Regression } \\
\hline Parameter & Estimate & ApproxStdErr \\
\hline A & -2.801823619 & 0.36173537 \\
\hline B & 6904.78796 & 575.945501 \\
\hline $\mathrm{C}$ & 234.43663463 & 32.7818745 \\
\hline
\end{tabular}

Graph of Fulcher Fit

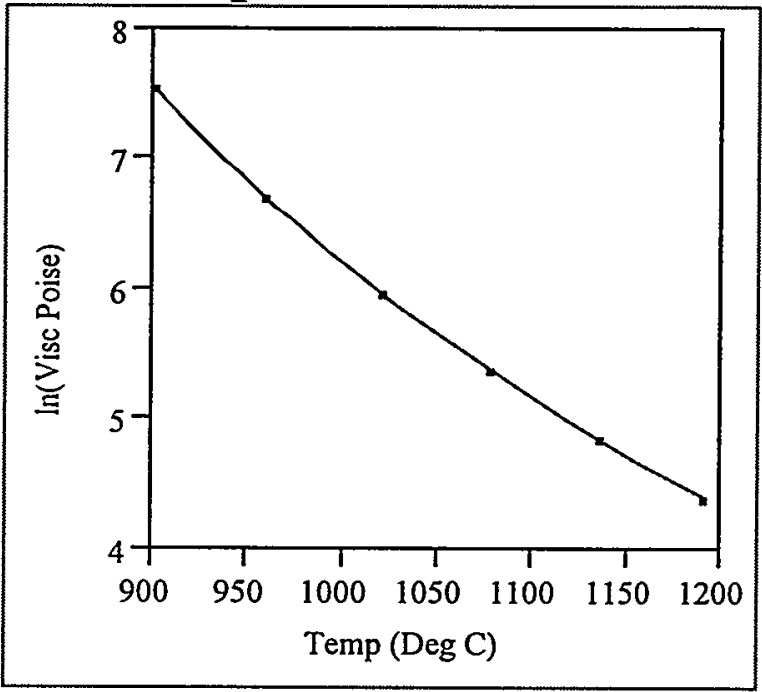

HM Measured by SS

\begin{tabular}{|c|c|c|c|c|}
\hline $\begin{array}{c}\text { Temp } \\
\text { (Deg C) }\end{array}$ & $\begin{array}{c}\text { Measured } \\
\text { Visc. } \\
\text { (Poise) }\end{array}$ & $\begin{array}{c}\text { In Visc } \\
\text { (Fulcher, } \\
\text { Poise) }\end{array}$ & $\begin{array}{c}\text { Measured } \\
\text { InVisc. } \\
\text { (Poise) }\end{array}$ & $\begin{array}{c}\text { Predicted } \\
\text { Visc. } \\
\text { (poise) }\end{array}$ \\
\hline 1192.53 & 81.33 & 4.404977 & 4.398515 & 81.86 \\
\hline 1136.36 & 128.57 & 4.853802 & 4.856474 & 128.23 \\
\hline 1079.07 & 218.56 & 5.37307 & 5.387061 & 215.52 \\
\hline 1021.47 & 389.61 & 5.97136 & 5.965146 & 392.04 \\
\hline 960.08 & 816.11 & 6.713578 & 6.704549 & 823.51 \\
\hline 901.78 & 1900.55 & 7.544856 & 7.549899 & 1890.99 \\
\hline $\mathbf{1 1 5 0}$ & $\mathbf{?}$ & $\mathbf{4 . 7 3 9 7 4 9}$ & $\mathbf{?}$ & $\mathbf{1 1 4 . 4 1}$ \\
\hline & & & & \\
\hline
\end{tabular}




\section{Sharp-Schurtz (SS)}

\section{Batch 1}

\begin{tabular}{lrr}
\multicolumn{3}{c}{ JMP® Nonlinear Regression } \\
Parameter & Estimate & ApproxStdErr \\
A & -3.032985109 & 0.28107546 \\
B & 6242.3592243 & 459.435771 \\
C & 235.02116276 & 29.5718562
\end{tabular}

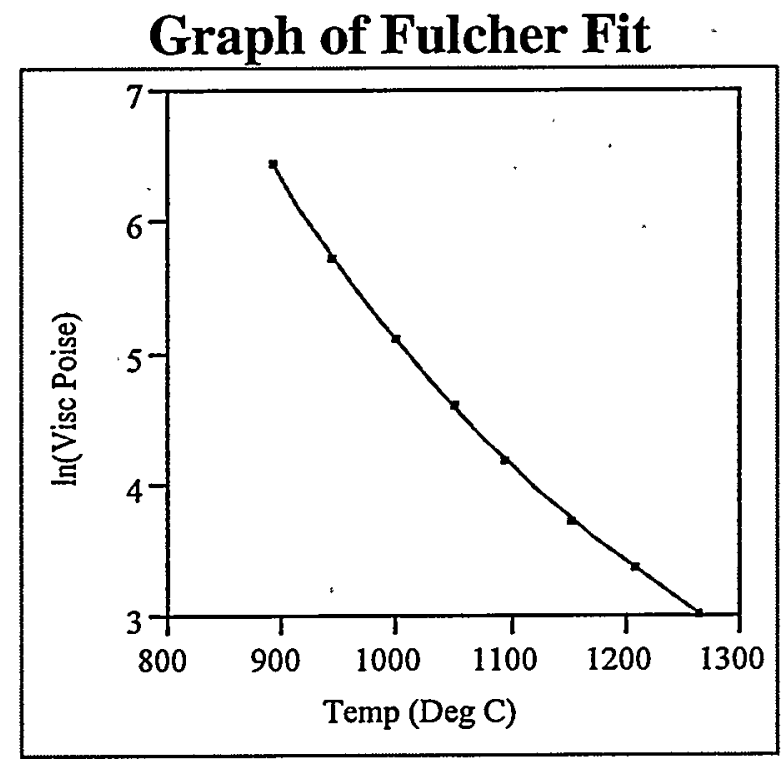

Batch 1 Measured by SS

\begin{tabular}{|c|c|c|c|c|}
\hline $\begin{array}{c}\text { Temp } \\
\text { (Deg C) }\end{array}$ & $\begin{array}{c}\text { Measured } \\
\text { Visc. } \\
\text { (Poise) }\end{array}$ & $\begin{array}{c}\text { In Visc } \\
\text { (Fulcher, } \\
\text { Poise) }\end{array}$ & $\begin{array}{c}\text { Measured } \\
\text { InVisc. } \\
\text { (Poise) }\end{array}$ & $\begin{array}{c}\text { Predicted } \\
\text { Visc. } \\
\text { (poise) }\end{array}$ \\
\hline 1264.72 & 20.85 & 3.02933 & 3.037354 & 20.68 \\
\hline 1207.85 & 29.4 & 3.383724 & 3.380995 & 29.48 \\
\hline 1153.8 & 42.85 & 3.761206 & 3.757706 & 43.00 \\
\hline 1094.46 & 67.25 & 4.230312 & 4.208417 & 68.74 \\
\hline 1050.26 & 103.01 & 4.624107 & 4.634826 & 101.91 \\
\hline 999.91 & 171.95 & 5.128147 & 5.147204 & 168.70 \\
\hline 945.56 & 312.32 & 5.752403 & 5.744028 & 314.95 \\
\hline 892.53 & 638.85 & 6.46097 & 6.45967 & 639.68 \\
\hline $\mathbf{1 1 5 0}$ & $\mathbf{?}$ & $\mathbf{3 . 7 8 9 4 2 3}$ & $\mathbf{?}$ & $\mathbf{4 4 . 2 3}$ \\
\hline & & & & \\
\hline
\end{tabular}




\section{Sharp-Schurtz (SS)}

\section{Purex}

\begin{tabular}{lrr}
\multicolumn{3}{c}{ JMP® ${ }^{\text {Nonlinear }}$ Regression } \\
Parameter & $\begin{array}{r}\text { Estimate } \\
\text { ApproxStdErr }\end{array}$ \\
A & -3.158433857 & 0.02522208 \\
B & 5390.4148385 & 35.2858111 \\
C & 274.39604438 & 2.24902056
\end{tabular}

Graph of Fulcher Fit

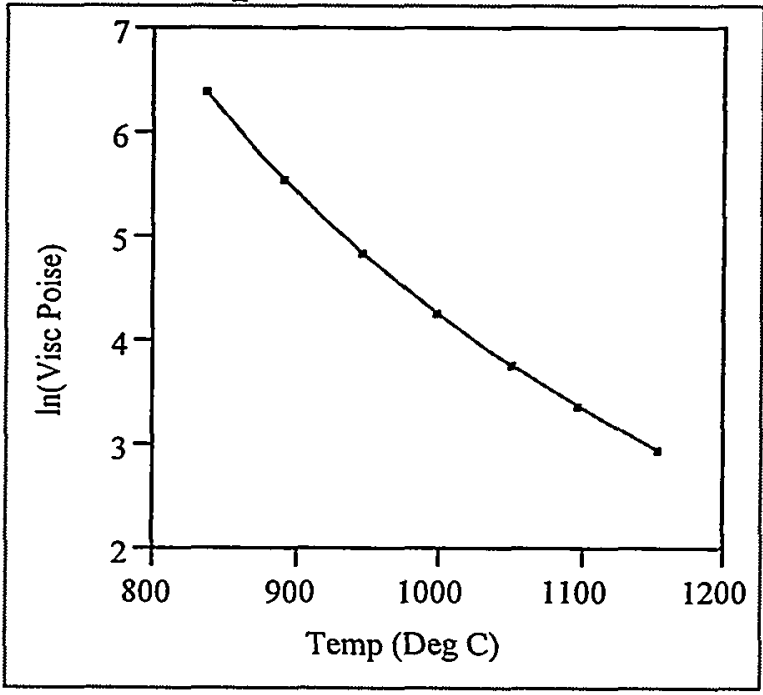

Purex Measured by SS

\begin{tabular}{|c|c|c|c|c|}
\hline $\begin{array}{c}\text { Temp } \\
\text { (Deg C) }\end{array}$ & $\begin{array}{c}\text { Measured } \\
\text { Visc. } \\
\text { (Poise) }\end{array}$ & $\begin{array}{c}\text { In Visc } \\
\text { (Fulcher, } \\
\text { Poise) }\end{array}$ & $\begin{array}{c}\text { Measured } \\
\text { lnVisc. } \\
\text { (Poise) }\end{array}$ & $\begin{array}{c}\text { Predicted } \\
\text { Visc. } \\
\text { (poise) }\end{array}$ \\
\hline 1155.32 & 19.33 & 2.960613 & 2.961658 & 19.31 \\
\hline 1097.62 & 29.61 & 3.389499 & 3.388112 & 29.65 \\
\hline 1051.16 & 43.85 & 3.781145 & 3.780775 & 43.87 \\
\hline 998.24 & 72.85 & 4.288496 & 4.288403 & 72.86 \\
\hline 946.17 & 129.97 & 4.865716 & 4.867304 & 129.76 \\
\hline 893 & 258.42 & 5.555404 & 5.554586 & 258.63 \\
\hline 838 & 605.34 & 6.405755 & 6.40579 & 605.32 \\
\hline $\mathbf{1 1 5 0}$ & $\mathbf{P}$ & $\mathbf{2 . 9 9 7 7 9 1}$ & $\mathbf{?}$ & $\mathbf{2 0 . 0 4}$ \\
\hline & & & & \\
\hline
\end{tabular}


Target \& Measured Compositions and Predicted \& Average Measured Viscosities at $1150{ }^{\circ} \mathrm{C}$

\begin{tabular}{|c|c|c|c|c|c|c|c|c|c|c|c|c|c|c|c|c|c|c|c|c|}
\hline \multirow{3}{*}{$\begin{array}{c}\text { SRTC } \\
\text { Glass ID }\end{array}$} & \multirow{2}{*}{\multicolumn{5}{|c|}{ Target Compositions as Weight Fractions }} & \multirow[b]{3}{*}{$\mathrm{Fe} 2 \mathrm{O} 3$} & \multirow[b]{3}{*}{$\mathrm{K} 2 \mathrm{O}$} & \multirow[b]{3}{*}{$\mathrm{Li} 20$} & \multirow[b]{3}{*}{$\mathrm{MgO}$} & \multirow[b]{3}{*}{ Mno } & \multirow[b]{3}{*}{$\mathrm{Na} 2 \mathrm{O}$} & \multirow[b]{3}{*}{$\mathrm{NiO}$} & \multirow[b]{3}{*}{$\mathrm{SiO} 2$} & \multirow[b]{3}{*}{$\mathrm{TiO} 2$} & \multirow{2}{*}{\multicolumn{3}{|c|}{$\begin{array}{l}\text { Predicted } \\
\text { Visc. }\end{array}$}} & \multirow{3}{*}{ CELS } & \multirow{4}{*}{$\begin{array}{c}\text { PNNL } \\
88.8\end{array}$} & \multirow{3}{*}{ SS } \\
\hline & & & & & & & & & & & & & & & & & & & & \\
\hline & Al2O3 & $\mathrm{B} 2 \mathrm{O} 3$ & $\mathrm{CaO}$ & $\mathrm{Cr} 2 \mathrm{O3}$ & $\mathrm{CuO}$ & & & & & & & & & & U308 & Poise & SRTC & & & \\
\hline Glass 1 & 0.0623 & 0.0850 & 0.0148 & 0.0010 & 0.0003 & 0.1291 & 0.0000 & 0.0342 & 0.0219 & 0.0159 & 0.1097 & 0.0022 & 0.5040 & 0.0010 & 0.0160 & 82.51 & 87.9 & & & \\
\hline Glass 2 & 0.0623 & 0.0850 & 0.0230 & 0.0013 & 0.0009 & 0.1291 & 0.0000 & 0.0342 & 0.0280 & 0.0185 & 0.1097 & 0.0020 & 0.5040 & 0.0020 & 0.0000 & 82.51 & 65.6 & 61.2 & & \\
\hline Glass 3 & 0.0605 & 0.0865 & 0.0139 & 0.0009 & 0.0003 & 0.1115 & 0.0000 & 0.0355 & 0.0209 & 0.0143 & 0.1096 & 0.0020 & 0.5177 & 0.0020 & 0.0160 & 92.884 & 102.5 & & 95.8 & \\
\hline Glass 4 & 0.0664 & 0.0853 & 0.0178 & 0.0010 & 0.0003 & 0.1115 & 0.0000 & 0.0333 & 0.0275 & 0.0188 & 0.1095 & 0.0020 & 0.5257 & 0.0010 & 0.0000 & 108.895 & 102.4 & 92.6 & & \\
\hline Glass 5 & 0.0664 & 0.0853 & 0.0140 & 0.0010 & 0.0003 & 0.1115 & 0.0000 & 0.0333 & 0.0200 & 0.0150 & 0.1095 & 0.0020 & 0.5257 & 0.0010 & 0.0150 & 108.895 & 108.4 & & 104.7 & \\
\hline Batch 1 & 0.0488 & 0.0777 & 0.0122 & 0.0011 & 0.0040 & 0.1284 & 0.0327 & 0.0443 & 0.0142 & 0.0173 & 0.0900 & 0.0075 & 0.5022 & 0.0067 & 0.0000 & 56.167 & 48.6 & & & 44.2 \\
\hline $\mathrm{HM}$ & 0.0715 & 0.0703 & 0.0101 & 0.0009 & 0.0025 & 0.0778 & 0.0221 & 0.0462 & 0.0149 & 0.0176 & 0.0856 & 0.0041 & 0.5579 & 0.0056 & 0.0000 & 137.244 & 121.7 & 111.5 & 121.5 & 114.4 \\
\hline Purex & 0.0299 & 0.1033 & 0.0109 & 0.0015 & 0.0042 & 0.1325 & 0.0341 & 0.0322 & 0.0141 & 0.0169 & 0.1262 & 0.0119 & 0.4654 & 0.0068 & 0.0000 & 24.832 & 22.5 & & & 20 \\
\hline Uranium Std & 0.0410 & 0.0921 & 0.0130 & 0.0030 & 0.0000 & 0.1320 & 0.0300 & 0.0306 & 0.0121 & 0.0289 & 0.1180 & 0.0112 & 0.4535 & 0.0105 & 0.0241 & 33.2 & 32.2 & & & \\
\hline $\begin{array}{c}\text { SRTC } \\
\text { Glass ID }\end{array}$ & & ured & mpositic & as a & ht Frac & Ions & & & & & & & & & & $\begin{array}{l}\text { Predicted } \\
\text { Visc. }\end{array}$ & & & & \\
\hline & $\mathrm{A} 12 \mathrm{O} 3$ & B2O3 & $\mathrm{CaO}$ & $\mathrm{Cr} 2 \mathrm{O} 3$ & $\mathrm{CuO}$ & $\mathrm{Fe} 2 \mathrm{O} 3$ & $\mathrm{~K} 2 \mathrm{O}$ & $\mathrm{Li} 20$ & $\mathrm{MgO}$ & $\mathrm{MnO}$ & $\mathrm{Na} 2 \mathrm{O}$ & $\mathrm{Ni}$ & $\mathrm{SiO} 2$ & $\mathrm{TiO} 2$ & $\mathrm{U} 308$ & Poise & SRTC & CELS & PNNL & SS \\
\hline Glass 1 & 0.0683 & 0.0892 & 0.0174 & 0.0008 & 0.0003 & 0.1320 & 0.0000 & 0.0343 & 0.0207 & 0.0160 & 0.1190 & 0.0022 & 0.4820 & 0.0014 & 0.0131 & 63.14 & 87.9 & & - & \\
\hline Glass 2 & 0.0648 & 0.0864 & 0.0232 & 0.0012 & 0.0009 & 0.1160 & 0.0000 & 0.0290 & 0.0241 & 0.0158 & 0.1060 & 0.0019 & 0.4790 & 0.0022 & 0.0000 & 94.003 & 65.6 & 61.2 & & \\
\hline Glass 3 & 0.0651 & 0.0880 & 0.0158 & 0.0007 & 0.0004 & 0.1030 & 0.0000 & 0.0373 & 0.0195 & 0.0139 & 0.1140 & 0.0019 & 0.5050 & 0.0022 & 0.0146 & 82.875 & 102.5 & & 95.8 & \\
\hline Glass 4 & 0.0685 & 0.0896 & 0.0195 & 0.0010 & 0.0003 & 0.0988 & 0.0000 & 0.0275 & 0.0242 & 0.0153 & 0.1130 & 0.0020 & 0.5120 & 0.0133 & 0.0000 & 119.752 & 102.4 & 92.6 & & \\
\hline Glass 5 & 0.0665 & 0.0867 & 0.0144 & 0.0008 & 0.0003 & 0.1010 & 0.0000 & 0.0343 & 0.0177 & 0.0143 & 0.1120 & 0.0019 & 0.5000 & 0.0012 & 0.0143 & 92.654 & 108.4 & & 104.7 & \\
\hline
\end{tabular}




\section{D.WPF Distribution}

M. N. Brosee, 704-S

R. E. Edwards, 704-25S

H. H. Elder, 704-S

D. C. Iverson, 704-30S

W. D. Kerley, 704-S

J. E. Occhipinti, 704-27S

J. F. Ortaldo, 704-S

J. W. Ray, 704-35S

\section{SRTC Distribution}

D. F. Bickford, 773-43A

K. G. Brown, 704-1T

A. D. Cozzi, 773-43A

D. A. Crowley, 773-43A

T. B. Edwards, 773-42A

C. R. Goetzman, 773-A

J. R. Harbour, 773-43A

E. W. Holtzscheiter, 773-A

C. M. Jantzen, 773-A

J. C. Marra, 773-43A

S. L. Marra, 704-T

L. M. Papouchado, 773-A

D. K. Peeler, 773-43A

R. F. Schumacher, 773-43A

M. E. Smith, 773-43A

R. H. Spires, 773-A

R. C. Tuckfield, 773-42A

J. R. Workman, 773-A 